\title{
Recognition Problems for Connectivity Functions
}

Susan Jowett

\section{VICTORIA UNIVERSITY OF WELLINGTON}

Te Whare Wānanga o te Ūpoko o te Ika a Māui

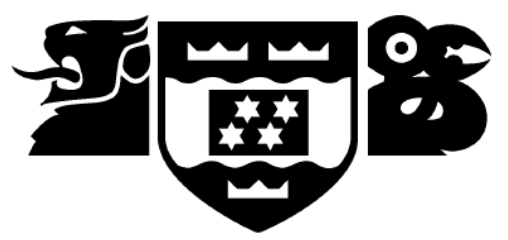

School of Mathematics, Statistics

and Operations Research

Te Kura Mātai Tatauranga, Rangahau Pūnaha

\section{A thesis}

submitted to the Victoria University of Wellington in fulfilment of the requirements for the degree of

Master of Science

in Mathematics.

Victoria University of Wellington 


\begin{abstract}
A connectivity function is a symmetric, submodular set function. Connectivity functions arise naturally from graphs, matroids and other structures. This thesis focuses mainly on recognition problems for connectivity functions, that is when a connectivity function comes from a particular type of structure. In particular we give a method for identifying when a connectivity function comes from a graph, which uses no more than a polynomial number of evaluations of the connectivity function. We also give a proof that no such method can exist for matroids.
\end{abstract}




\section{Acknowledgements}

I would like to thank my amazing supervisor Geoff Whittle for his help and support throughout the year. I would also like to thank my parents for their proof-reading skills and patience, particularly my father, who I made read and understand my thesis and pick up my mistakes, and who also made me many programs to check submodularity.

Thanks to my office-mates, both official and unofficial: Jasmine, Giovanna, Matt, and Harpreet, for helping me procrastinate so much throughout the year.

Finally I would like to thank the matroid group at Victoria, particularly Ben and Michael My LTEX Monkey. 


\section{Contents}

1 Introduction 1

2 Basic Results 5

2.1 Examples $\ldots \ldots \ldots \ldots$

2.2 Minors of Connectivity Functions . . . . . . . . . . . . . . . . 10

2.3 Direct Sum . . . . . . . . . . . . . . . . . . . . . . . . . . . . 13

2.4 Connected Matroids and Graphs. . . . . . . . . . . . . . . . . 15

\begin{tabular}{lll}
\hline 3 & Polymatroids & 18
\end{tabular}

3.1 Examples $\ldots \ldots \ldots \ldots$

3.2 Connectivity Functions and Polymatroids . . . . . . . . . . . . 20

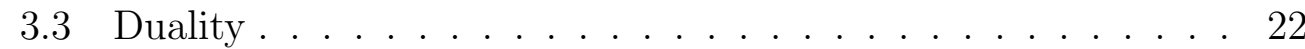

3.4 Minors . . . . . . . . . . . . . . . . . . . . . . . . . . . . . . . . 28

$3.5 \quad$ 2-sums and Unavoidable Squidge-Minors of 2-polymatroids . . 45

4 Graphic Connectivity Functions 50

$4.1 \quad$ Facts About Connectivity Functions of Graphs . . . . . . . . . 52

4.2 Building a Graph from the Edge Adjacencies . . . . . . . . . . 62

4.3 When Is a Connectivity Function Graphic? . . . . . . . . . . . 71

5 Matroidal Connectivity Functions 100

5.1 Matroids With The Same Connectivity Function . . . . . . . . 102 
5.2 Connectivity Polymatroids . . . . . . . . . . . . . . . . . . 119

5.3 Spikes . . . . . . . . . . . . . . . . . . . . . . . . 121

5.4 Self-Dual Matroids . . . . . . . . . . . . . . . . . . . . . . . 128

$\begin{array}{lll}6 & \text { Branch-Width } & 134\end{array}$

6.1 Main Branch-Width Theorem . . . . . . . . . . . . . . . . . . 142 


\section{Chapter 1}

\section{Introduction}

A number of mathematical structures, such as graphs, matroids, and polymatroids, have an associated set function that captures connectivity in these structures. All these functions have certain properties in common, namely they are all symmetric and submodular. It turns out that these are often the only properties we need to prove theorems about the connectivity of these structures. This motivates the study of connectivity functions in general, where we define a connectivity function to be a symmetric, submodular set function.

A polymatroid is similar to a matroid, except that it may have elements $e$ such that $r(e)>1$, where $r$ is the rank function of the polymatroid. A halfintegral polymatroid is a polymatroid such that $r(A) \in\left\{\frac{n}{2}: n \in \mathbb{Z}_{\geq 0}\right\}$. In [8] the following result is proved:

Theorem 1.1. Let $\lambda$ be a connectivity function of $E$. Then there is a halfintegral polymatroid, $P$, on $E$ such that $\lambda=\mu_{P}$ where $\mu_{P}$ is the connectivity function of $P$.

This motivates us to study polymatroids in detail later on, in the hopes of developing better insight into the structure of connectivity functions. In our study we develop a notion of duality for polymatroids and a new minor operation, which corresponds to a natural minor operation in connectivity functions. We spend some time investigating the relationship between the notion of polymatroid duality given here and this minor operation. This work extends work done in [8]. 
The main aim of this thesis is to study recognition problems for connectivity functions, that is identifying when a connectivity function comes from a particular class of mathematical structures. There are two different types of such problems that we study here. The first is recognising when a connectivity function comes from some particular structure in a polynomial number of evaluations of the connectivity function, where, when we talk about a polynomial number of evaluations of the connectivity function, we mean polynomial in the size of the set on which the connectivity function is based. As this is the main type of recognition problem that we study, to simplify things, this interpretation of polynomial number will be used throughout the thesis; that is when we talk about a polynomial number of evaluations of the connectivity function, we shall be talking about polynomial in the size of the set on which the connectivity function is based. The other type of recognition problem we look briefly at is adding an extra axiom to the axioms of connectivity functions to get an axiomatization of when a connectivity function comes from a particular structure. Say we are considering the question of when a connectivity function is matroidal. We can, of course, always add an axiom to the axioms of a connectivity function that states for a connectivity function, $\lambda$ "There exists a matroid $M$ such that the connectivity function of $M$ is equal to $\lambda$ ". This ensures that there is some matroid with connectivity function $\lambda$, but it also does not seem like a particularly satisfactory axiom. What we are really interested in is the question of whether there is an axiom that can be written in the language in which the axioms of connectivity functions are written, which, when added to the axioms of connectivity functions, gives an axiomatization of when a connectivity function comes from a particular structure. A discussion of a somewhat similar problem for matroids can be found in [7].

In Chapter 4 we show that the connectivity function of a graph gives a lot of information about the structure of the graph with that connectivity function. In fact, we show that, from the connectivity function of a graph, we can determine the edge adjacencies. We then give a method for building the graph (almost uniquely) from the edge adjacencies. In fact, building the graph given a graphic connectivity function requires only a polynomial number of rank evaluations. The main result of this thesis is the following:

Theorem 4.1. There is some polynomial p such that, given an arbitrary 
connectivity function $\lambda$, we are able to establish whether or not $\lambda$ is graphic in at most $p(n)$ evaluations of the connectivity function, where $n$ is the size of the set on which $\lambda$ is based.

We prove this by first building the graph that would have $\lambda$ as its connectivity function, were $\lambda$ graphic. To do this we pretend that $\lambda$ is graphic and use the method for building the graph given a graphic connectivity function. We then show that if $\lambda$ and the connectivity function, $\gamma$, of the graph that we just built agree on some particular collection of subsets of $E$ (of which there will be a polynomial number), then $\lambda$ and $\gamma$ agree everywhere, and so $\lambda$ is graphic.

This result is reminiscent of results of Seymour [12] and Lemos [6] (the latter result we shall later go into in some detail), which show that connectivity function of a matroid tell us a lot about the structure of that matroid and, in all but a few cases, determines, up to duality, the structure of the matroid.

In Chapter 5 we look at the aforementioned result of Lemos, from [6], which states that if two matroids, $M_{1}$ and $M_{2}$, have the same connectivity function, and $r\left(M_{1}\right) \neq r\left(M_{2}\right)$, then $M_{2}=M_{1}^{*}$. This result shows that, in some sense, the connectivity function of a matroid in many cases almost uniquely identifies the matroid. As this is an interesting and important result we reprove it here. The proof is very similar to the one given in [6], but uses slightly more elementary results. We also give a slight generalisation of a result from [8], that gives a description of when a connectivity polymatroid (a particular type of polymatroid which we construct from a connectivity function), is the connectivity polymatroid of a connectivity function that comes from a matroid. We then prove the following result:

Theorem 5.34. We cannot tell in a polynomial number of evaluations of the connectivity function, if a connectivity function is matroidal.

To prove this we look to a class of matroids known as spikes, which are known to provide counterexamples to many natural conjectures. Finally we take a slightly different approach and look at adding extra axioms to the axioms of connectivity function that identify when a connectivity function is the connectivity function of an identically self-dual matroid. Unfortunately the methods used to do this in this special case do not seem to generalise obviously into a method for doing this for matroids in general. 
We also take a detour and consider branch-width of connectivity functions. We prove that the class of excluded minors for connectivity functions of bounded branch-width have bounded size. The proof of this closely follows [2], which gives a bound on the size excluded minors of branch-width $k$. However, in the case of connectivity functions, we think the bound on size that actually exists is much better than the one I have. 


\section{Chapter 2}

\section{Basic Results}

In this section we introduce connectivity functions, give some example of connectivity functions, and give some basic results that will be used in later sections.

\section{Definition 2.1.}

1. Let $E$ be a finite set, an integer-valued set function on $E$ is a function from the power set of $E$, denoted $2^{|E|}$ to some subset of $\mathbb{Z}$.

2. An integer-valued set function $f$ on $E$ is normalised if $f(\emptyset)=0$.

3. An integer-valued set function $f$ on $E$ is symmetric if $f(X)=f(E-X)$ for all $X \subseteq E$.

4. An integer-valued set function $f$ on $E$ is submodular if $f(X)+f(Y) \geq$ $f(X \cup Y)+f(X \cap Y)$ for all $X, Y \subseteq E$.

5. A normalised integer-valued set function $f$ on $E$ is unitary if $f(e) \leq 1$ for all $e \in E$.

Definition 2.2. An integer-valued set function $\mu: 2^{|E|} \rightarrow \mathbb{Z}$ is a connectivity function if the following hold:

(C1) $\mu$ is normalised

(C2) $\mu$ is symmetric

(C3) $\mu$ is submodular. 
Definition 2.3. We say that a connectivity function $\lambda$ is connected if $\lambda(X) \neq$ 0 for all non-empty $X \subsetneq E$. If $\lambda$ is not connected then we say that $\lambda$ is disconnected. If $\lambda(X)=0$ for some non-empty $X \subsetneq E$, then we say that $X$ is a separator of $\lambda$, and we say that $X$ is a connected component of $\lambda$ if $X$ is a minimal non-empty separator of $\lambda$.

\subsection{Examples}

The following examples of connectivity functions arise naturally from mathematical structures. They are well-known and can be found in [9] and [8] for example. These objects and their connectivity functions will be studied in more detail in later sections.

\section{Matroids}

Definition 2.4. A matroid $M$ is a pair $(E, r)$, where $E$ is a finite set and $r$ is an integer-valued set function on $E$ having the following properties:

(R1) $r$ is normalised.

(R2) $r(X) \leq|X|$, for all $X \subseteq E$.

(R3) $r(X) \leq r(Y)$, for all $X \subseteq Y \subseteq E$ ( $r$ is increasing).

(R4) $r$ is submodular.

We define the connectivity function of a matroid as follows:

Definition 2.5. Let $M=(E, r)$. Define an integer-valued set function on E by

$$
\mu(X)=r(X)+r(E-X)-r(E)
$$

for $X \subseteq E$. Then $\mu$ is the connectivity function of the matroid $M$.

A proof that the connectivity function of a matroid is a unitary connectivity function can be found in [8]

We will also define the notion of a dual of a matroid: 
Definition 2.6. Let $M=(E, r)$ be a matroid and let $r^{*}$ be a integer-valued set function on $E$ defined by $r^{*}(X)=|X|+r(E-X)-r(E)$ for all $X \subseteq E$. Define the dual of $M$ to be $M^{*}=\left(E, r^{*}\right)$.

It is a well-known result and can be found in [9] that the dual of a matroid is a matroid and if $M$ is a matroid, then $M^{* *}=M$. It is also clear from the definition of the connectivity function of a matroid and the definition of dual that, for a matroid $M=(E, r)$, we have $\mu(X)=r(X)+r^{*}(X)-|X|$ for all $X \subseteq E$, and therefore, the connectivity function of $M$ and the connectivity function of the dual of $M$ are equal.

Sometimes a matroid, $M=(E, r)$, can be represented by a matrix, $A$, over a field $\mathbb{F}$, whose columns are labelled by the elements of $E$, where $X \subseteq$ $E$ is independent if, and only if, the columns labelled by $X$ are linearly independent in $\mathbb{F}$. This means that the rank of a subset, $X$, of $E$ is given by the dimension of the span of $X$. When we can represent a matroid in such a way we say that it is representable. If $M$ is representable, then $\mu_{M}(X)=r(\langle X\rangle \cap\langle E-X\rangle)$ for all $X \subseteq E$. That is the connectivity of a subset, $X$, of $E$ is the dimension of the span of the intersection of the spans of $X$ and $E-X$.

\section{Graphs}

Definition 2.7. A graph consists of a set $V(G)$ of vertices, a set $E(G)$ of edges, and an incidence relation $\varphi: E \rightarrow 2^{|V|}$ such that $|\varphi(\{e\})| \in\{1,2\}$. We say that two vertices, $v_{i}$ and $v_{j}$, are adjacent if there is some $e \in E$ such that $\varphi(\{e\})=\left\{v_{i}, v_{j}\right\}$; and we say that an edge, $e$, is incident with a vertex, $v$, if $v \in \varphi(\{e\})$.

Let $X \subseteq E$. We use $V(X)$ to denote the collection of vertices of $G$ that are incident with some edge in $X$. We define the connectivity function of a graph as follows:

Definition 2.8. Let $G$ be a graph with vertex set $V(G)$ and edge set $E(G)$. The graphic connectivity function of $G$, denoted by $\gamma$, is defined by

$$
\gamma(X)=|V(X)|+|V(E-X)|-|V(E)|
$$


for all $X \subseteq E(G)$

A proof that a graphic connectivity function is indeed a connectivity function can be found in [8]. It is easy to see that for $X \subseteq E$, the connectivity, $\gamma(X)$, is equal to the number of vertices that $X$ and $E-X$ have in common (i.e. $|V(X) \cap V(E-X)|)$.

Note that the connectivity function of the graph as defined above does not agree with the connectivity function of the graphic matroid. For example consider the following graph, $G$ :

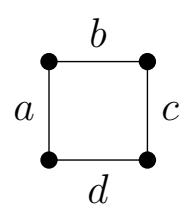

This has graphic connectivity function $\gamma_{G}$ as follows:

$\gamma_{G}(\{a\})=2, \gamma_{G}(\{b\})=2, \gamma_{G}(\{c\})=2, \gamma_{G}(\{d\})=2, \gamma_{G}(\{a, b\})=2$, $\gamma_{G}(\{a, c\})=4, \gamma_{G}(\{a, d\})=2, \gamma_{G}(\{b, c\})=2, \gamma_{G}(\{b, d\})=4, \gamma_{G}(\{c, d\})=2$ and the matroid connectivity of the cycle matroid of the graph, denoted $\mu_{M(G)}$, is as follows:

$\mu_{M(G)}(\{a\})=1, \mu_{M(G)}(\{b\})=1, \mu_{M(G)}(\{c\})=1, \mu_{M(G)}(\{d\})=1$, $\mu_{M(G)}(\{a, b\})=1, \mu_{M(G)}(\{a, c\})=1, \mu_{M(G)}(\{a, d\})=1, \mu_{M(G)}(\{b, c\})=1$, $\mu_{M(G)}(\{b, d\})=1, \mu_{M(G)}(\{c, d\})=1$.

In fact it is not even the case that a matroid connectivity function of the cycle matroid of the graph will be connected if the graph is connected. Trees, as graphs, have a connected graphic connectivity function, but viewed as cycle matroids the matroidal connectivity function of the cycle matroid is not connected.

The graphic connectivity function defined above is also known as the vertex connectivity of $G$, and when we talk about the connectivity function of a graph, this is the connectivity function we mean. However, this is not the only connectivity function we can get from a graph and we now define another connectivity function which comes from a graph, this connectivity function is discussed in [1] for example. It is given here as another example of a connectivity function, and to show that we can define more than one type of connectivity function on a graph; it will not be used again in this thesis. 
Let $G=(V, E)$ be a graph with $V$ as its collection of vertices and $E$ as its collection of edges. Let $X \subseteq V$. Let $\lambda_{V(G)}(X)$ be the number of edges incident with both a vertex in $X$ and a vertex in $E-X$. It is clear that $\lambda_{V(G)}$ is symmetric and normalised. To see that $\lambda_{V(G)}$ is submodular, consider $\lambda_{V(G)}(X)+\lambda_{V_{G}}(Y)$ for some $X, Y \subseteq V$. As $\lambda_{V(G)}(X)$ is equal to the number of edges incident with both a vertex in $X$ and a vertex in $E-X$ we can say that $\lambda_{V(G)}(X)=|E(X)|+|E(V-X)|-|E|$, where we use $E(X)$ to denote the edges incident with a vertex in $X$. Therefore:

$$
\begin{aligned}
\lambda_{V(G)}(X)+\lambda_{V(G)}(Y) & =|E(X)|+|E(V-X)|+|E(Y)|+|E(V-Y)|-2|E(G)| \\
& =|E(X) \cup E(Y)|+|E(X) \cap E(Y)|+\mid E(V-X) \cup \\
& E(V-Y)|+| E(V-X) \cap E(V-Y)|-2| E(G) \mid \\
& \geq|E(X \cup Y)|+|E(X \cap Y)|+|E((V-X) \cup(V-Y))| \\
& +|E((V-X) \cap(V-Y))|-2|E(G)| \\
& =|E(X \cup Y)|+|E(X \cap Y)|+|E(V-(X \cap Y))| \\
& +|E(V-(X \cup Y))|-2|E(G)| \\
& =\lambda_{V(G)}(X \cup Y)+\lambda_{V(G)}(X \cap Y)
\end{aligned}
$$

where (1) follows by the principle of inclusion exclusion, (2) follows as $\mid E(X) \cup$ $E(Y)|=| E(X \cup Y) \mid$ and $E(X) \cap E(Y) \supseteq E(X \cap Y)$. Therefore $\lambda_{V(G)}$ is submodular. As it is also normalised and symmetric, $\lambda_{V(G)}$ is a connectivity function.

\section{Polymatroids}

Definition 2.9. A polymatroid $P$ is a pair $(E, r)$, where $E$ is a finite set and $r$ is a rational valued set function on $E$ having the following properties:

(R1) $r$ is normalised.

(R2) $r(X) \leq r(Y)$, for all $X \subseteq Y \subseteq E$

(R3) $r$ is submodular.

This is the same as the definition of a matroid, except that $r(X)$ is not required to be less than or equal to $|X|$, and need not be integer-valued. We shall sometimes use $r(P)$ to denote $r_{P}(E)$. 
We say that a polymatroid $P=(E, r)$ is integer-valued if $r(X) \in \mathbb{Z}$ for all $X \subseteq E$.

We define the connectivity function of a polymatroid as follows:

Definition 2.10. Let $P=(E, r)$. Define a set function on $E$ by

$$
\lambda_{P}(X)=r(X)+r(E-X)-r(P)
$$

for $X \subseteq E$. Then $\lambda_{P}$ is the connectivity function of the polymatroid, $\mathrm{P}$.

In fact the connectivity function of a polymatroid is not necessarily a connectivity function, as $\lambda_{P}(X)$ may not be integer-valued for all $X$. However, it is proved in [8] that a connectivity function of a polymatroid is normalised, increasing and submodular. Therefore we can say that the connectivity function of a polymatroid, $P=(E, r)$, is a connectivity function if, and only if, $\lambda_{P}(X) \in \mathbb{Z}$ for all $X \subseteq E$. Therefore the connectivity function of an integer-valued polymatroid is a connectivity function. We can also have the connectivity functions of polymatroids which are not integer polymatroids, that are still connectivity functions. One such example is given in [8] and another is given here: Let $E=\{a, b, c, d\}$ and the rank function be defined as follows:

$$
r(X)=\left\{\begin{array}{cl}
1 & \text { if }|X|=1 \\
\frac{3}{2} & |X|=2 \\
2 & \text { otherwise }
\end{array}\right.
$$

Then

$$
\lambda_{P}(X)= \begin{cases}1 & \text { if }|X|=1 \text { or }|X|=2 \text { or }|X|=3 \\ 0 & \text { if }|X|=0 \text { or }|X|=4\end{cases}
$$

\subsection{Minors of Connectivity Functions}

This section gives an operation that removes a single element from a connectivity function but still preserves some of the structure of the original connectivity function. It is studied for unitary connectivity functions in detail in [8], and we generalise those results to general connectivity functions here.

Definition 2.11. Let $\mu$ be a connectivity function on $E$ and let $e \in E$. The 
elision of $e$ from $\lambda$, which we denote by $\lambda \downarrow e$, is defined as follows:

$$
(\lambda \downarrow e)(A)=\min \{\lambda(A), \lambda(A \cup\{e\})\}
$$

for all $A \subseteq E-\{e\}$.

Note that for all $A \subseteq E-\{e\}$, we have $(\lambda \downarrow e)(A) \leq \lambda(A)$ and $(\lambda \downarrow e)(A) \leq$ $\lambda(A \cup\{e\})$.

Theorem 2.12. Let $\lambda$ be connectivity function on set $E$ and let $e \in E$; then $\lambda \downarrow e$ is a connectivity function on the set $E-\{e\}$.

Proof. It is easy to see that $(\lambda \downarrow e)(\emptyset)=0$.

Let $\{A, e, B\}$ be a partition of $E$. Then:

$$
\begin{aligned}
(\lambda \downarrow e)(A) & =\min \{\lambda(A), \lambda(A \cup\{e\})\} \\
& =\min \{\lambda(B \cup\{e\}), \lambda(B)\} \\
& =(\lambda \downarrow e)(B) .
\end{aligned}
$$

Therefore $\lambda \downarrow e$ is symmetric.

To show submodularity consider $A, B \subseteq E-\{e\}$. By definition $(\lambda \downarrow e)(A)+$ $\lambda \downarrow(B)=\min \{\lambda(A), \lambda(A \cup\{e\})\}+\min \{\lambda(B), \lambda(B \cup\{e\})\}$. We shall now split into four cases:

Suppose that $(\lambda \downarrow e)(A)=\lambda(A)$ and $(\lambda \downarrow e)(B)=\lambda(B)$. Then

$$
\begin{aligned}
(\lambda \downarrow e)(A)+(\lambda \downarrow e)(B) & =\lambda(A)+\lambda(B) \\
& \geq \lambda(A \cup B)+\lambda(A \cap B) \\
& \geq(\lambda \downarrow e)(A \cup B)+(\lambda \downarrow e)(A \cap B) .
\end{aligned}
$$

Suppose that $(\lambda \downarrow e)(A)=\lambda(A \cup\{e\})$, and $(\lambda \downarrow e)(B)=\lambda(B)$. Then

$$
\begin{aligned}
(\lambda \downarrow e)(A)+(\lambda \downarrow e)(B) & =\lambda(A \cup\{e\})+\lambda(B) \\
& \geq \lambda(A \cup B \cup\{e\})+\lambda(A \cap B) \\
& \geq(\lambda \downarrow e)(A \cup B)+(\lambda \downarrow e)(A \cap B) .
\end{aligned}
$$

If $(\lambda \downarrow e)(A)=\lambda(A)$, and $(\lambda \downarrow e)(B) \neq \lambda(B)$, then the proof is similar. 
Finally, suppose that $(\lambda \downarrow e)(A)=\lambda(A \cup\{e\})$ and $(\lambda \downarrow e)(B)=\lambda(B \cup\{e\})$. Then

$$
\begin{aligned}
(\lambda \downarrow e)(A)+(\lambda \downarrow e)(B) & =\lambda(A \cup\{e\})+\lambda(B \cup\{e\}) \\
& \geq \lambda((A \cap B) \cup\{e\})+\lambda((A \cup B) \cup\{e\}) \\
& \geq(\lambda \downarrow e)(A \cap B)+(\lambda \downarrow e)(A \cup B) .
\end{aligned}
$$

Therefore submodularity holds in all cases. As $\lambda \downarrow e$ is symmetric and submodular and normalised, it must be that $\lambda \downarrow e$ is a connectivity function.

Clearly if we have a connectivity function that has the property that $\lambda(\{a\}) \leq$ $k$ for all $a \in E$, then we also have this property for $\lambda \downarrow e$ for any $e \in E$. In particular if $\lambda$ is a unitary connectivity function, then so is $\lambda \downarrow e$.

Lemma 2.13. Let $\lambda$ be a connectivity function on a set $E$ and let $e \in E$. Let $X \subseteq E-\{e\}$ be such that $\lambda(X \cup\{e\}) \leq \lambda(X)$. Then for any $X^{\prime} \supseteq X$ we have $\lambda\left(X^{\prime} \cup\{e\}\right) \leq \lambda\left(X^{\prime}\right)$.

Proof. Suppose that $\lambda\left(X^{\prime} \cup\{e\}\right)>\lambda\left(X^{\prime}\right)$. Then:

$$
\begin{aligned}
\lambda\left(X^{\prime} \cup\{e\}\right)+\lambda(X \cup\{e\}) & >\lambda\left(X^{\prime}\right)+\lambda(X \cup\{e\}) \\
& \geq \lambda\left(X^{\prime} \cup\{e\}\right)+\lambda(X) \\
& \geq \lambda\left(X^{\prime} \cup\{e\}\right)+\lambda(X \cup\{e\})
\end{aligned}
$$

where the first inequality holds by our supposition, (2) follows from (1) by submodularity and (3) follows from (2) by the assumption in the statement of the lemma. Therefore $\lambda\left(X^{\prime} \cup\{e\}\right)+\lambda(X \cup\{e\})>\lambda\left(X^{\prime} \cup\{e\}\right)+\lambda(X \cup\{e\})$, a contradiction.

Lemma 2.14. Suppose that $(\lambda \downarrow e)(X)=\lambda(X \cup\{e\})$, then for any $X^{\prime} \supset X$, we have $(\lambda \downarrow e)\left(X^{\prime}\right)=\lambda\left(X^{\prime} \cup\{e\}\right)$.

Proof. If $(\lambda \downarrow e)(X)=\lambda(X \cup\{e\})$, then $\lambda(X \cup\{e\}) \leq \lambda(X)$. Therefore, by the previous lemma, $\lambda\left(X^{\prime} \cup\{e\}\right) \leq \lambda\left(X^{\prime}\right)$ for any $X^{\prime} \subseteq E$ that is a superset of $X$. Therefore $\left.(\lambda \downarrow e)\left(X^{\prime}\right)=\min \left\{\lambda\left(X^{\prime}\right), \lambda\left(X^{\prime} \cup\{e\}\right)\right\}\right)=\lambda\left(X^{\prime} \cup\{e\}\right)$. 
By symmetry we can say that if $(\lambda \downarrow e)(X)=\lambda(X)$, then for any $X^{\prime} \subseteq X$, we have $(\lambda \downarrow e)\left(X^{\prime}\right)=\lambda\left(X^{\prime}\right)$.

\subsection{Direct Sum}

Recall that a connectivity function $\lambda$ is connected if $\lambda(X) \neq 0$ for all nonempty $X \subsetneq E$. If $\lambda$ is not connected then we say that $\lambda$ is disconnected. If $\lambda(X)=0$ for some non-empty $X \subsetneq E$, then we say that $X$ is a separator of $\lambda$, and we say that $X$ is a connected component of $\lambda$ if $X$ is a minimal non-empty separator of $\lambda$.

Definition 2.15. Let $\lambda_{1}$ and $\lambda_{2}$ be connectivity functions on sets $E_{1}$ and $E_{2}$ respectively, such that $E_{1} \cap E_{2}=\emptyset$. The direct-sum of $\lambda_{1}$ and $\lambda_{2}$, denoted $\lambda_{1} \oplus \lambda_{2}$, is defined as follows:

$$
\lambda_{1} \oplus \lambda_{2}(X)=\lambda_{1}\left(X \cap E_{1}\right)+\lambda_{2}\left(X \cap E_{2}\right),
$$

for all $X \subseteq E_{1} \cup E_{2}$.

In [8] it is proved that the direct-sum of two connectivity functions is a connectivity function on $E_{1} \cup E_{2}$. The following lemma can also be found in [8].

Lemma 2.16. Let $\lambda$ be a connectivity function on a set $E$, such that $\lambda(X)=$ 0 for some non-empty proper subset, $X$, of $E$. Then there are connectivity functions $\lambda_{1}$ on $X$ and $\lambda_{2}$ on $E-X$ such that $\lambda=\lambda_{1} \oplus \lambda_{2}$. Conversely if $\lambda=\lambda_{1} \oplus \lambda_{2}$, where $\lambda_{1}$ is a connectivity function on set $X$, and $\lambda_{2}$ is a connectivity function on set $E-X$, then $\lambda(X)=0$.

Proof. For the first part, suppose $\lambda(X)=0$, and define $\lambda_{1}$ on set $X$ such that $\lambda_{1}(A)=\lambda(A)$ for all $A \subseteq X$.

Claim 2.16.1. $\lambda_{1}$ is a connectivity function

Proof. $\lambda_{1}(\emptyset)=\lambda(\emptyset)=0$, so $\lambda_{1}$ is normalised.

Let $A$ and $B$ be subsets of $X$. Then $\lambda_{1}(A)+\lambda_{1}(B)=\lambda(A)+\lambda(B) \geq$ $\lambda(A \cup B)+\lambda(A \cap B)=\lambda_{1}(A \cup B)+\lambda_{1}(A \cap B)$. Therefore $\lambda_{1}$ is submodular. 
Let $A \subseteq X$. Then $\lambda_{1}(A)=\lambda(A)=\lambda(E-A)=\lambda((E-X) \cup(X-A))$. Therefore, as $\lambda(E-X)=0$, by submodularity of $\lambda$,

$\lambda_{1}(X-A)=\lambda(E-X)+\lambda(X-A) \geq \lambda((E-X) \cup(X-A))=\lambda_{1}(A)$,

Therefore $\lambda(X-A)=\lambda_{1}(X-A) \geq \lambda_{1}(A)$. By symmetry and submodularity of $\lambda$,

$$
\lambda(X)+\lambda_{1}(A)=\lambda(X)+\lambda(E-A) \geq \lambda(E)+\lambda(X-A),
$$

and therefore $\lambda_{1}(A) \geq \lambda_{1}(X-A)$. Combining the two inequalities shows that $\lambda_{1}(A)=\lambda_{1}(X-A)$, and so $\lambda_{1}$ is symmetric.

Similarly we can define a connectivity function $\lambda_{2}$ on $E-X$ such that $\lambda_{2}(B)=$ $\lambda(B)$ for all $B \subseteq E-X$.

Consider $\lambda(Y)$ for any $Y \subseteq E$, and let $Y_{1}=Y \cap X$ and $Y_{2}=Y \cap(E-X)$. By submodularity $\lambda_{1}\left(Y_{1}\right)+\lambda_{2}\left(Y_{2}\right)=\lambda\left(Y_{1}\right)+\lambda\left(Y_{2}\right) \geq \lambda\left(Y_{1} \cup Y_{2}\right)=\lambda(Y)$. We also have:

$$
\begin{aligned}
\lambda(Y) & =\lambda(Y)+\lambda(X)+\lambda(E-X) \\
& \geq \lambda\left(Y_{1}\right)+\lambda\left(X \cup Y_{2}\right)+\lambda(E-X) \\
& \geq \lambda\left(Y_{1}\right)+\lambda(E)+\lambda\left(Y_{2}\right) \\
& =\lambda_{1}\left(Y_{1}\right)+\lambda_{2}\left(Y_{2}\right),
\end{aligned}
$$

where (2) follows from (1) by submodularity applied to $\lambda(Y)+\lambda(X)$ and (3) follows from (2) by submodularity applied to $\lambda\left(X \cup Y_{2}\right)$ and $\lambda(E-X)$.

Therefore $\lambda(Y)=\lambda\left(Y_{1}\right)+\lambda\left(Y_{2}\right)=\lambda_{1}\left(Y_{1}\right)+\lambda_{2}\left(Y_{2}\right)$ so $\lambda=\lambda_{1} \oplus \lambda_{2}$.

For the converse, suppose that $\lambda=\lambda_{1} \oplus \lambda_{2}$, where $\lambda_{1}$ is a connectivity function on set $X$ and $\lambda_{2}$ is a connectivity function on $E-X$. Then $\lambda(X)=$ $\lambda_{1}(X)=0$.

We would like our minor operation to be able to 'pick out' the connected components of a connectivity function. The following lemma shows that the elision operation can indeed do this.

In [8] it is proved that $(\lambda \downarrow e) \downarrow f=(\lambda \downarrow f) \downarrow e$. Therefore it makes sense to 
talk about $\lambda \downarrow A$ for some $A \subseteq E$. It can easily be seen that

$$
(\lambda \downarrow A)(X)=\min \{\lambda(X \cup C): C \subseteq A\}
$$

Lemma 2.17. Let $\lambda$ be a connectivity function on a set $E$ and let $\emptyset \neq X \subsetneq E$ be such that $\lambda(X)=0$. Let $Y=E-X$. Then $(\lambda \downarrow Y)(A)=\lambda(A)$ for all $A \subseteq X$

Proof. As $\lambda(X)=0$ we can find connectivity functions, $\lambda_{1}$ on set $X$ and $\lambda_{2}$ on set $E-X$, such that $\lambda=\lambda_{1} \oplus \lambda_{2}$. We want to show that $(\lambda \downarrow Y)(A)=\lambda(A)$ for all $A \subseteq X$, in other words, $\min \{\lambda(A \cup C)$ for all $C \subseteq Y\}=\lambda(A)$. This holds as $\lambda(A \cup C)=\lambda_{1}(A)+\lambda_{2}(C) \geq \lambda_{1}(A)=\lambda(A)$.

\subsection{Connected Matroids and Graphs}

Ideally we would like the connectivity function of a structure to be connected if, and only if, that structure is connected. We show that this is true for graphs and matroids.

\subsubsection{Graphs}

In 9] a graph is said to be connected, if each pair of distinct vertices is joined by a path, otherwise it is said to be disconnected. A connected component of $G$ is a maximal connected subgraph of $G$. We show that a graph is connected if, and only if, its vertex connectivity function is connected. Suppose that $G$ is not connected. Then $G$ must have at least two connected components. Call one of these $G_{A}$ and let $E\left(G_{A}\right)=A$. As there is no walk from any vertex of $G_{A}$ to any vertex in the complement of $G_{A}$, we can say that $V(A) \cap$ $V(E-A)=\emptyset$, and therefore $V(A)+|V(E-A)|=|V(E)|$, and so $\gamma_{G}(A)=$ $|V(A)|+|V(E-A)|-|V(E)|=0$.

Now suppose that $\gamma_{G}(A)=0$ for some $\emptyset \neq A \neq E$. Then $|V(A)|+\mid V(E-$ $A)|=| V(E) \mid$, and so $V(A) \cap V(E-A)=\emptyset$. We shall show that $A$ induces a connected component, or collection of connected components of $G$. For suppose not, then for every $v \in V(A)$, there is a path between $v$ and some $u \in V(E-A)$. Chose $u$ so that this path is of minimal length, and suppose 
the path is $v, e_{1}, v_{1}, e_{2}, v_{2}, \ldots, e_{n}, v_{n}, e_{n+1}, u$. If $e_{n+1} \in A$, then $u \in V(A)$, so $V(A) \cap V(E-A) \neq \emptyset$, a contradiction. Therefore $e_{n+1} \notin A$, so $e_{n+1} \in E-A$, and so $v_{n} \in V(E-A)$ contradicting the choice of $u$.

\subsubsection{Matroids}

Definition 2.18. Let $M_{1}=\left(E_{1}, r_{1}\right)$ and $M_{2}=\left(E_{2}, r_{2}\right)$ be matroids such that $E_{1} \cap E_{2}=\emptyset$. We define the direct sum of $M_{1}$ and $M_{2}$ by $M_{1} \oplus M_{2}=$ $\left(E_{1} \cup E_{2}, r_{M_{1} \oplus M_{2}}\right)$ where $r_{M_{1} \oplus M_{2}}$ is defined as follows:

$$
r_{M_{1} \oplus M_{2}}(X)=r_{1}\left(X \cap E_{1}\right)+r_{2}\left(X \cap E_{2}\right),
$$

for all $X \subseteq E_{1} \cup E_{2}$.

It is easily verified that $M_{1} \oplus M_{2}$ is a matroid.

Definition 2.19. We say that a matroid, $M$, is disconnected if there is some partition $(A, B)$ of the groundset of $M$ such that there is no circuit of $M$ intersecting both $A$ and $B$. Otherwise we say that $M$ is connected.

We could also say that a matroid, $M$, is connected if, and only if, there is no pair of matroids $M_{1}$ and $M_{2}$ such that $M=M_{1} \oplus M_{2}$.

We use $M \mid A$ to denote the matroid $M \backslash(E-A)$.

Lemma 2.20. A matroid $M=\left(E, r_{M}\right)$ is connected if, and only if, its connectivity function, $\mu_{M}$ is connected.

Proof. Suppose that $M$ is connected, then, for each non-trivial partition, $(A, B)$, of the groundset of $M$, there is some circuit $C$ that has a non-empty intersection with both $A$ and $B$. This means that $r(A \cap C)+r(B \cap C)-r(C)=$ 1. Now consider $r(A)+r(B)+r(C)$,

$$
\begin{aligned}
r(A)+r(B)+r(C) & \geq r(A \cap C)+r(A \cup C)+r(B) \\
& \geq r(A \cap C)+r((A \cup C) \cup B))+r((A \cup C) \cap B) \\
& =r(A \cap C)+r(E)+r(C \cap B)
\end{aligned}
$$

where the first and second inequalities follow by submodularity and (3) follows from (2) by noting that $A \cup B=E$ and that $A \cap B=\emptyset$. Therefore 
$r(A)+r(B)+r(C) \geq r(A \cap C)+r(B \cap C)+r(E)$, and so $r(A)+r(B)-r(E) \geq$ $r(A \cap C)+r(B \cap C)-r(C)=1$. This means $\mu_{M}(A) \neq 0$. As this holds for all partitions $(A, B)$ of $E$, the connectivity function is non-zero on all non-empty proper subsets of $E$, and therefore $\mu_{M}$ is connected.

Now suppose that $M$ is not connected. Then there is some non-trivial partition, $(X, Y)$ of $E$ such that no circuit of $M$ has a non-empty intersection with both $X$ and $Y$. Let $B_{X}$ be a base of $M \mid X$ and $B_{Y}$ be a base of $M \mid Y$. As neither $B_{X}$ nor $B_{Y}$ contains a circuit of $M$ and $B_{X} \cup B_{Y}$ does not contain a circuit of $M$, the set $B_{X} \cup B_{Y}$ must be independent in $M$. Consider $\mu_{M}(X)$ :

$$
\begin{aligned}
\mu_{M}(X) & =r(X)+r(Y)-r(E) \\
& =\left|B_{X}\right|+\left|B_{Y}\right|-r(E) \\
& \leq\left|B_{X}\right|+\left|B_{Y}\right|-\left|B_{X} \cup B_{Y}\right| \\
& =0
\end{aligned}
$$

Therefore when $M$ is not connected there is some $\emptyset \neq X \neq E$ such that $\mu(X)=0$.

This means that if $\mu(X)=0$, then there is no circuit intersecting both $X$ and $E-X$. This fact will be used later, in Section 5 . 


\section{Chapter 3}

\section{Polymatroids}

In [8] it is proved that every connectivity function is the connectivity function of a half-integral polymatroid. This leads us to study polymatroids in some detail and, as part of that study, to generalise some of the results given in [8].

Recall that a polymatroid is an ordered pair $P=\left(E, r_{P}\right)$ where $E$ is a finite set and $r_{P}$ is a non-negative rational valued set function on $E$ which has the following properties:

(P1) $r_{P}(\emptyset)=0\left(r_{P}\right.$ is normalised $)$,

(P2) If $A \subseteq B \subseteq E$ then $r_{P}(A) \leq r_{P}(B)$ ( $r_{P}$ is increasing),

(P3) If $A, B \subseteq E$ then $r_{P}(A)+r_{P}(B) \geq r_{P}(A \cup B)+r_{P}(A \cap B)\left(r_{P}\right.$ is submodular).

If $P=\left(E, r_{P}\right)$ is a polymatroid, then we call $r_{P}$ the rank function of $P$ and we define the rank of the polymatroid, which we denote $r(P)$, by $r(P)=r_{P}(E)$. Throughout this section we shall let $P=\left(E, r_{P}\right)$ be a polymatroid.

When trying to visualise a polymatroid it can be helpful to think of rank-0 elements as loops, rank-1 elements as points, rank-2 elements as lines etc. Therefore we shall sometimes refer to rank-1 elements as points, rank-2 elements as lines etc.

We do not require the rank of an element to be an integer, which leads to the following definitions: 


\section{Definition 3.1.}

i) An integer polymatroid is one in which $r_{P}: 2^{E} \rightarrow \mathbb{Z}_{\geq 0}$.

ii) A half-integral polymatroid is one in which $r_{P}(A) \in\left\{\frac{n}{2}: n \in \mathbb{Z}_{\geq 0}\right\}$ for all $A \subseteq E$.

Definition 3.2. A k-polymatroid is a polymatroid $P=\left(E, r_{P}\right)$ such that for each $e \in E$ we have $r_{P}(\{e\}) \leq k$. A $k$-polymatroid is a pure $k$-polymatroid if for every $e \in E$ we have $r_{P}(\{e\})=k$.

We will briefly look at a few examples of polymatroids.

\subsection{Examples}

\section{Graphic Polymatroids}

Suppose we have a graph $G$. Define $P(G)=(E(G), r)$ where $r(X)=|V(X)|$ for every $X \subseteq E(G)$. It can be seen that $P(G)$ is a 2-polymatroid, and when $G$ is a loopless graph this is a pure 2-polymatroid. A proof of this can be found in [8].

\section{Polymatroids that come from matroids}

i) Clearly a matroid is just a 1-polymatroid.

ii) Suppose we have a matroid $M$ with groundset $E^{\prime}$ and rank function $r^{\prime}$. Define $P=(E, r)$ where $E$ is some subset of the set of flats of $M$ of rank at most $k$ and $r(X)=r^{\prime}\left(\bigcup_{x \in X} x\right)$ for all $X \subseteq E$. This is a $k$-polymatroid and every integer-valued $k$-polymatroid can be obtained in this way. A sketch of the proof of this can be found in [9].

iii) Let $M_{1}=\left(E, r_{1}\right)$ and $M_{2}=\left(E, r_{2}\right)$ be matroids. Define $M_{1}+M_{2}=$ $\left(E, r_{1}+r_{2}\right)$, where $\left(r_{1}+r_{2}\right)(X)=r_{1}(X)+r_{2}(X)$. It is easy to see that this is a 2-polymatroid. Intuitively the two matroids are embedded in disjoint dimensions, and corresponding points are then joined by a line which becomes an element of the polymatroid. The points of the matroid are then deleted. 


\subsection{Connectivity Functions and Polyma- troids}

We now look at some results relating to the connectivity functions of polymatroids.

Recall that the connectivity function of a polymatroid, $\mu_{P}(X)$, is defined by

$$
\mu_{P}(X)=r_{P}(X)+r_{P}(E-X)-r(P)
$$

for all $X \subseteq E$. A proof that the connectivity function of a polymatroid is indeed a connectivity function can be found in [8]. When the polymatroid comes from a graph, $G$, then $\mu_{P}=\gamma$, where $\gamma$ is the connectivity function of $G$. When a polymatroid is a matroid then the polymatroid connectivity and the matroid connectivity agree.

Throughout the remainder of this section we shall assume that $\mu_{P}$ is the connectivity function of the polymatroid $P=\left(E, r_{P}\right)$.

Definition 3.3. We say that $P$ is connected if $r_{P}(X)+r_{P}(E-X)>r(P)$ for all non-empty $X \subsetneq E$

This is equivalent to saying that $\mu_{P}(X) \neq 0$ for all non-empty $X \subsetneq E$. Note that is $P$ is connected if, and only if, $\mu_{P}$ is connected.

Definition 3.4. Let $e \in E$.

i) $e$ is compact if $r_{P}(\{e\})=\mu_{P}(\{e\})$.

ii) A set $X \subseteq E$ is called compact if every element $x \in X$ is compact.

iii) $P$ is called compact if every element of $P$ is compact.

Lemma 3.5. The element $e$ is a compact element of $E$ if, and only if, $r_{P}(E-$ $\{e\})=r_{P}(E)$.

Proof. If $e$ is compact then $r_{P}(\{e\})=\mu_{P}(\{e\})=r_{P}(\{e\})+r_{P}(E-\{e\})-$ $r_{P}(E)$. Therefore $r_{P}(E-\{e\})=r_{P}(E)$. The reverse direction is similar.

A coloop-free matroid, $M$, is a compact 1-polymatroid. For, if $e$ is not a coloop then there is some basis, $B$, of $M$ that does not contain $e$ and so 
$e \in \operatorname{cl}(B)$. Therefore $r(E-\{e\})=r(E)$ and so $e$ is compact for every element, $e$, of $M$. A connected matroid on a groundset containing at least two elements is coloop-free as, if $e$ were a coloop $r(E-\{e\})=r(E)-1$ and so $r(E-\{e\})+r(\{e\})=r(E)$, a contradiction to the connectedness of $M$. Therefore a connected matroid is compact.

In [8] it is proved that if $\mu_{P}$ is the connectivity function of a polymatroid $P$, then there is a compact polymatroid $P^{\prime}$ whose connectivity function is also $\mu_{P}$. The following very nice result (mentioned earlier) is also proved and we include the proof for the sake of completeness. Before we do this we introduce the following notation:

Suppose that we have a connectivity function $\lambda$ on a set $E$. Then for any $X \subseteq E$, we define $\|X\|_{\lambda}$ as follows:

$$
\|X\|_{\lambda}=\sum_{x \in X} \lambda(\{x\})
$$

Theorem 3.6. Let $\lambda$ be a connectivity function on a set $E$. Then there is a half-integral polymatroid, $P=\left(E, r_{P}\right)$, such that $\mu_{P}=\lambda$.

Proof. The proof follows by constructing the rank function of $P$.

Define $r_{P}$ by:

$$
r_{P}(X)=\frac{\lambda(X)+\|X\|_{\lambda}}{2}
$$

for all $X \subseteq E$.

It is not hard to see that $r_{P}$ is the rank function of a polymatroid. This polymatroid is clearly half-integral as both $\lambda(X)$ and $\|X\|_{\lambda}$ are integers.

Now consider the connectivity function, $\mu_{P}$, of $P$.

$$
\begin{aligned}
\mu_{P}(X) & =r_{P}(X)+r_{P}(E-X)-r_{P}(E) \\
& =\frac{1}{2}\left(\lambda(X)+\|X\|_{\lambda}+\lambda(E-X)+\|E-X\|_{\lambda}-\lambda(E)-\|E\|_{\lambda}\right) \\
& =\frac{1}{2}\left(\lambda(X)+\|X\|_{\lambda}+\lambda(X)+\|E\|_{\lambda}-\|X\|_{\lambda}-\lambda(E)-\|E\|_{\lambda}\right) \\
& =\frac{1}{2}(2 \lambda(X)) \\
& =\lambda(X) .
\end{aligned}
$$


This motivates the following definition:

Definition 3.7. Let $\lambda$ be a connectivity function on $E$. Then the polymatroid $P_{\lambda}$, with rank function $r_{P_{\lambda}}$ defined by:

$$
r_{P_{\lambda}}(X)=\|X\|_{\lambda}+\lambda(X)
$$

for all $X \subseteq E$, is called the connectivity polymatroid generated by $\lambda$.

We say that $P$ is a connectivity polymatroid, if we can find some connectivity function $\lambda$ such that $r_{P}=r_{P_{\lambda}}$.

\subsection{Duality}

It is proved in [8] that a pure 2-polymatroid, $P$, is the connectivity polymatroid generated by a unitary connectivity function if, and only if, $P=P^{*}$, where, for a 2 -polymatroid $P$, the polymatroid $P^{*}$ is defined by

$$
r_{P^{*}}(X)=2|X|+r_{P}(E-X)-r_{P}(E)
$$

The definition of duality given above is an example of the $k$-dual of a matroid, which is defined in [16]. The $k$-dual of a $k$-polymatroid, $P$, is a polymatroid on the same groundset of $P$ with rank function defined as follows:

$$
r_{P^{*}}(X)=k|X|+r_{P}(E-X)-r_{P}(E)
$$

In [16] Whittle proved that this is the only definition of duality in polymatroids that interchanges deletion and contraction and has the property that $P=P^{* *}$. However, this definition of duality relies on the value of $k$, and, as a 2-polymatroid is also a 3-polymatroid, this means that the dual is not uniquely defined. Another problem is that the 2-dual of a 3-polymatroid is not even a polymatroid. We introduce a new notion of duality which also gives a fairly nice relationship between deletion and contraction and agrees with the previous definition of duality when $P$ is a pure, compact 2-polymatroid. This means that, with our new definition of duality, we are 
still able to say that a pure 2-polymatroid, $P$, is the connectivity polymatroid generated by a unitary connectivity function if, and only if, $P=P^{*}$. We are also able to extend this characterisation to general (that is, not necessarily unitary) connectivity polymatroids. Before we do this we show that our definition of duality is a reasonable definition.

If $P$ is a polymatroid on set $E$ with rank function $r_{P}$, then we define

$$
\|X\|_{r_{P}}=\sum_{x \in X} r_{P}(\{x\})
$$

for all $X \subseteq E$. Note that if $P$ is a compact polymatroid with connectivity function $\mu$, then $\|X\|_{r}=\|X\|_{\mu}$.

Definition 3.8. Let $P=(E, r)$ be a polymatroid; we define a function $r_{P^{*}}$ on $E$ as follows:

$$
r_{P^{*}}(X)=r(E-X)+\|X\|_{r}-r(E)
$$

for all $X \subseteq E$. We call the pair $P^{*}=\left(E, r_{P^{*}}\right)$ the dual of $P$.

It is not hard to see that when $P$ is a pure, compact $k$-polymatroid, then the definition of the $k$-polymatroid dual agrees with the dual given in Definition 3.8. The dual defined in Definition 3.8 will be the polymatroid dual that we use throughout the remainder of this thesis.

Lemma 3.9. Suppose we have a polymatroid $P=\left(E, r_{P}\right)$ with dual $P^{*}=$ $\left(E, r_{P^{*}}\right)$. Then $P^{*}$ is a polymatroid on $E$ with rank function $r_{P^{*}}$.

Proof. If $X=\emptyset$ then $r_{P^{*}}(X)=r_{P^{*}}(\emptyset)=r_{P}(E-\emptyset)+\|\emptyset\|_{r_{P}}-r_{P}(E)=0$, in other words $r_{P^{*}}$ is normalised.

Suppose that $X \subseteq Y \subseteq E$ such that $X \cup\{e\}=Y$ for $e \in E-X$, and let $r_{P}(\{e\})=k$ for some $k$. By the definition of dual $r_{P^{*}}(X)=r_{P}(E-X)+$ $\|X\|_{r_{P}}-r_{P}(E)$, in other words, $r_{P^{*}}(X)=r_{P}(E-X)+\|Y\|_{r_{P}}-k-r_{P}(E)$. As $E-X=(E-Y) \cup\{e\}$, it follows by submodularity of $r$, that $r_{P}(E-X) \leq$ $r_{P}(E-Y)+r_{P}(\{e\})$. Therefore, $r_{P^{*}}(X) \leq r_{P}(E-Y)+k+\|Y\|_{r_{P}}-k-$ $r_{P}(E)=r_{P^{*}}(Y)$. Therefore, inductively, if $X \subseteq Y$ then $r_{P^{*}}(X) \leq r_{P^{*}}(Y)$, that is $r_{P^{*}}$ is increasing. 
Suppose $X, Y \subseteq E$ and consider $r_{P^{*}}(X)+r_{P^{*}}(Y)$. By definition of $r_{P^{*}}$ we know that $r_{P^{*}}(X)+r_{P^{*}}(Y)=r_{P}(E-X)+\|X\|_{r_{P}}-r_{P}(E)+r_{P}(E-$ $Y)+\|Y\|_{r_{P}}-r_{P}(E)$ which, by submodularity, is greater than or equal to $r_{P}((E-X) \cup(E-Y))+r_{P}((E-X) \cap(E-Y))+\|X\|_{r_{P}}+\|Y\|_{r_{P}}-2 r_{P}(E)$. It is clear that $\|X\|_{r_{P}}+\|Y\|_{r_{P}}=\|X \cup Y\|_{r_{P}}+\|X \cap Y\|_{r_{P}}$, and so $r_{P^{*}}(X)+$ $r_{P^{*}}(Y) \geq r_{P}(E-(X \cap Y))+r_{P}(E-(X \cup Y))+\|X \cup Y\|_{r_{P}}+\|X \cap Y\|_{r_{P}}-$ $2 r_{P}(E)=r_{P^{*}}(X \cup Y)+r_{P^{*}}(X \cap Y)$. In other words $r_{P^{*}}$ is submodular.

$P^{*}=\left(E, r_{P^{*}}\right)$ is normalised, increasing and submodular, and so $P^{*}$ is a polymatroid.

Lemma 3.10. The dual of $P$ is a compact polymatroid.

Proof. By the definition of the connectivity function of a polymatroid, $\mu_{P^{*}}(\{e\})=r_{P^{*}}(\{e\})+r_{P^{*}}(E-\{e\})-r_{P^{*}}(E)$; and, by the definition of $r_{P^{*}}$, we know that $r_{P^{*}}(E-\{e\})=r_{P}(E-(E-\{e\}))+\|E-\{e\}\|_{r_{P}}-r_{P}(E)$ and $r_{P^{*}}(E)=r_{P}(E-E)+\|E\|_{r_{P}}-r_{P}(E)$. Substituting into the formula for $\mu_{P^{*}}(\{e\})$ we get $\mu_{P^{*}}(\{e\})=r_{P^{*}}(\{e\})+r_{P}(\{e\})+\|E\|_{r_{P}}-r_{P}(\{e\})-r_{P}(E)-$ $\|E\|_{r_{P}}+r_{P}(E)=r_{P^{*}}(\{e\})$

It is also worth noting the following two lemmas:

Lemma 3.11. A polymatroid $P$ is connected if, and only if, $P^{*}$ is connected.

Proof. Suppose $P$ is connected, then for all non-empty $X \subsetneq E$ we have $r_{P}(X)+r_{P}(E-X)>r_{P}(E)$. Therefore:

$$
\begin{aligned}
r_{P^{*}}(X)+r_{P^{*}}(E-X)= & r_{P}(E-X)+\|X\|_{r}-r_{P}(E)+r_{P}(X) \\
& \quad+\|E-X\|_{r}-r_{P}(E) \\
= & r_{P}(X)+r_{P}(E-X)+\|E\|_{r}-2 r_{P}(E) \\
> & r_{P}(E)+\|E\|_{r}-2 r_{P}(E) \\
= & \|E\|_{r}-r_{P}(E) \\
= & r_{P^{*}}(E) .
\end{aligned}
$$

A similar argument works to show that if $P$ is not connected then $P^{*}$ is not connected.

Lemma 3.12. For every $e \in E$, the rank of e in the dual, $r_{P^{*}}(\{e\})$, is equal to $\mu_{P}(\{e\})$. Moreover, when e is compact, $r_{P^{*}}(\{e\})=r_{P}(\{e\})$. 
Proof. By the definition of duality of a polymatroid, $r_{P^{*}}(\{e\})=r_{P}(E-$ $\{e\})+r_{P}(\{e\})-r_{P}(E)$ which is just the definition of $\mu_{P}(\{e\})$. When $P$ is compact $\mu_{P}(\{e\})=r_{P}(\{e\})$, so $r_{P^{*}}(\{e\})=r_{P}(\{e\})$.

Therefore, if $e$ is a compact element of $E$ that corresponds to a point in $P$, it will also correspond to a point in $P^{*}$. Similarly if $e$ corresponds to a line in $P$, it will correspond to a line in $P^{*}$ etc. This means that this concept of polymatroid duality does not correspond to the standard concept of duality in a matroid as, in matroid duality, the dual of a loop (a rank-0 element) is a point (a rank-1 element).

We shall show that the dual of the dual of a compact polymatroid is the polymatroid itself. However, by Lemma 3.10, the dual of a non-compact polymatroid is a compact polymatroid and so, as the dual of the dual of a non-compact polymatroid is compact, it therefore cannot be the original polymatroid. We shall see that the dual of the dual of a non-compact polymatroid is what is known as the "compactification" of the polymatroid.

Definition 3.13. Let $r_{b(P)}$ be a function defined on $2^{E}$ as follows:

$$
r_{b(P)}(X)=r_{P}(X)+\sum_{x \in X}\left(\mu_{P}(x)-r_{P}(x)\right)
$$

for all $X \subseteq E$. The pair $b(P)=\left(E, r_{b(P)}\right)$ is the compactification of $P$.

The next lemma is a direct consequence of [8] Lemma 5.3.

Lemma 3.14. $b(P)$ is a compact polymatroid with connectivity function $\mu_{P}$.

Note that when $X$ is compact $\mu_{P}(\{x\})=r_{P}(\{x\})$ for all $x \in X$ so $r_{b(P)}(X)=$ $r_{P}(X)$. To give an intuitive idea of compactification we consider the following 2-polymatroids and their compactifications.

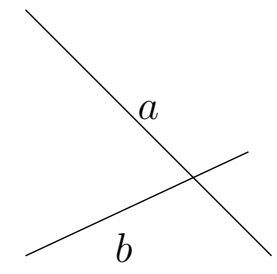

$P_{1}$

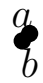

$b\left(P_{1}\right)$ 


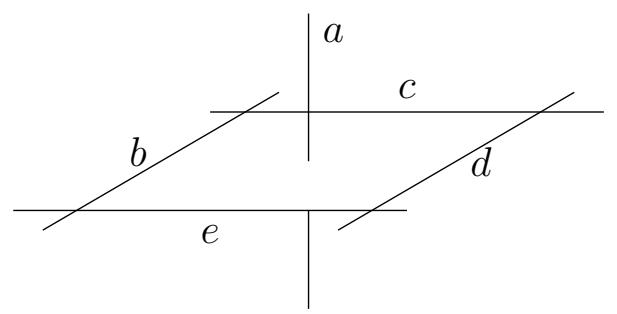

$P_{2}$

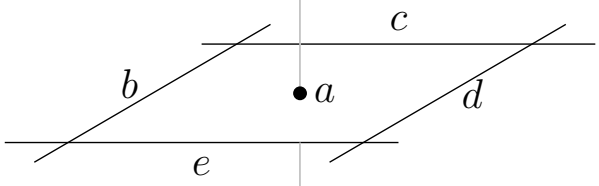

$b\left(P_{2}\right)$

We also consider the dual of $P_{1}$. It can easily be seen that the dual of $P_{1}$ is equal to $b\left(P_{1}\right)$, and the dual of $b\left(P_{1}\right)$ is $b\left(P_{1}\right)$ so $\left(P_{1}^{*}\right)^{*}=b\left(P_{1}\right)$, the compactification of $P_{1}$.

Lemma 3.15. The polymatroid $P$ is connected if, and only if, $b(P)$ is connected.

The proof is immediate from the definition of compactification.

Lemma 3.16. Let $P=\left(E, r_{P}\right)$ be a polymatroid. Then $\left(P^{*}\right)^{*}=b(P)$.

Proof. Consider $r_{\left(P^{*}\right)^{*}}(X)$ for $X \subseteq E$. Then

$$
\begin{aligned}
r_{\left(P^{*}\right)^{*}}(X)= & r_{P^{*}}(E-X)+\|X\|_{r_{P^{*}}}-r_{P^{*}}(E) \\
= & r_{P}(E-(E-X))+\|E-X\|_{r_{P}}-r_{P}(E)+\|X\|_{r_{P^{*}}} \\
& \quad-r_{P}(E-E)-\|E\|_{r_{P}}+r_{P}(E) \\
= & r_{P}(X)-\|X\|_{r_{P}}+\|X\|_{r_{P^{*}}} \\
= & r_{P}(X)+\sum_{x \in X}\left(\mu_{P}(\{x\})-r_{P}(\{x\})\right) \\
= & r_{b(P)}(X),
\end{aligned}
$$

where (1) follows by Lemma 3.12 .

The above results show that the dual polymatroid behaves in a reasonable way, so we now look at a characterisation of connectivity polymatroids. Recall that a connectivity polymatroid is a polymatroid $P$ such that $r_{P}(X)=\|X\|_{\lambda}+\lambda(X)$ for some connectivity function $\lambda$.

Theorem 3.17. A polymatroid $P=(E, r)$ is a connectivity polymatroid if, and only if, $P=P^{*}$ and $r_{P}(\{e\})$ is even for all $e \in E$. 
Proof. Suppose that $P=(E, r)$ is a connectivity polymatroid. Then $r_{P}(X)=\|X\|_{\lambda}+\lambda(X)$ for some connectivity function $\lambda$ on $E$. Therefore for any $e \in E$ we have $r_{P}(\{e\})=\|\{e\}\|_{\lambda}+\lambda(\{e\})=2 \lambda(\{e\})$, and so $r_{P}(\{e\})$ is even.

Next we must show that $P=P^{*}$. To do this note $r_{P}(E-X)=\| E-$ $X\left\|_{\lambda}+\lambda(E-X)=\right\| E-X \|_{\lambda}+\lambda(X)$, where the second equality follows by symmetry of the connectivity function; and $r_{P}(E)=\|E\|_{\lambda}+\lambda(E)=\|E\|_{\lambda}$. Therefore:

$$
\begin{aligned}
r_{P^{*}}(X) & =r_{P}(E-X)+\|X\|_{r_{P}}-r_{P}(E) \\
& =\|E-X\|_{\lambda}+\lambda(X)+\|X\|_{r_{P}}-\|E\|_{\lambda} \\
& =\|E\|_{\lambda}-\|X\|_{\lambda}+\lambda(X)+\|X\|_{r_{P}}-\|E\|_{\lambda} \\
& =\lambda(X)-\|X\|_{\lambda}+\|X\|_{r_{P}} \\
& =\lambda(X)+\sum_{x \in X}\left(r_{P}(\{x\})-\lambda(\{x\})\right) \\
& =\lambda(X)+\sum_{x \in X}(2 \lambda(\{x\})-\lambda(\{x\})) \\
& =\lambda(X)+\|X\|_{\lambda} \\
& =r_{P}(X) .
\end{aligned}
$$

where (1) follows as $P$ is a connectivity polymatroid.

For the converse suppose that $P=P^{*}$, and that $r_{P}(\{e\})$ is even for all $e \in E$. Let the connectivity function of $P$ be $\mu_{P}$. By rearranging the definition of the rank of the dual polymatroid we get $r_{P}(E-X)-r_{P}(E)=r_{P^{*}}(X)-\|X\|_{r_{P}}$, and therefore:

$$
\begin{aligned}
\mu_{P}(X) & =r_{P}(X)+r_{P}(E-X)-r_{P}(E) \\
& =r_{P}(X)+r_{P^{*}}(X)-\|X\|_{r_{P}} \\
& =2 r_{P}(X)-\|X\|_{r_{P}}
\end{aligned}
$$

As $r_{P}(\{e\})$ is even for all $e \in E$, we also know that $\|X\|_{r_{P}}$ is even for any $X \subseteq E$. Therefore $\mu(X)$ is even for all $X \subseteq E$, and so $\lambda=\frac{\mu}{2}$ is a connectivity function. As $\lambda=\frac{\mu}{2}$, for any $e \in E$ we have $\lambda(\{e\})=r_{P}(\{e\})-\frac{1}{2}\|\{e\}\|_{r_{P}}=$ 
$\frac{1}{2} r_{P}(\{e\})$. Rearranging this we get $r_{P}(\{e\})=2 \lambda(\{e\})$. Therefore:

$$
\begin{aligned}
\lambda(X) & =\frac{\mu(X)}{2} \\
& =r_{P}(X)-\frac{1}{2}\|X\|_{r_{P}} \\
& =r_{P}(X)-\frac{1}{2} \sum_{x \in X} r_{P}(x) \\
& =r_{P}(X)-\sum_{x \in X} \frac{1}{2} 2 \lambda(x) \\
& =r_{P}(X)-\|X\|_{\lambda} .
\end{aligned}
$$

Therefore $r_{P}(X)=\lambda(X)+\|X\|_{\lambda}$ and so $P=(E, r)$ is a connectivity polymatroid.

\subsection{Minors}

In this section we study deletion and contraction in polymatroids. We also introduce a new minor operation, which we call "squidging". If $P$ is a polymatroid obtained from a polymatroid $P^{\prime}$ by a series of deletions and contractions, then we call $P$ a minor of $P^{\prime}$. If $P$ is a polymatroid obtained from $P^{\prime}$ by a series of deletions, contractions, and squidging, then we say that $P$ is a squidge-minor of $P^{\prime}$. We prove that in a connected polymatroid we can find some squidge-minor operation that preserves connectedness. What is more, in a compact, connected polymatroid, almost all squidges preserve connectedness, although, in general, deletion and contraction do not. We also study the relationship between minor operations under duality.

Definition 3.18. Define the polymatroid $P \backslash A$, for every $A \subseteq E$, (which we call $P$ delete $A$ ) by $P \backslash A=\left(E-A, r_{P \backslash A}\right)$ where $r_{P \backslash A}$ is defined as follows:

$$
r_{P \backslash A}(B)=r_{P}(B)
$$

for each $B \subseteq E-A$. We also define the polymatroid $\left.P / A=(E-A), r_{P / A}\right)$ (known as $P$ contract $A$ ) where $r_{P / A}$ is defined as follows:

$$
r_{P / A}(B)=r_{P}(A \cup B)-r_{P}(A)
$$


From [16] we know that $P \backslash A$ and $P / A$ are indeed polymatroids, but it could be that $P \backslash A$ is not a compact polymatroid, even when $P$ is compact. For example, let $P$ be the compact 2-polymatroid below:

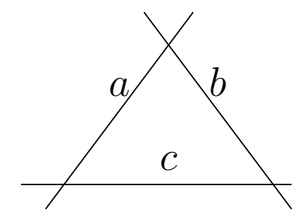

This has $r_{P}(\{d\})=2$ and $r_{P}(\{d, e\})=3$ for $d, e \in\{a, b, c\}$ and $r_{P}(\{a, b, c\})=$ 3. It can be seen that this polymatroid is compact.

The polymatroid $P \backslash a$ is shown below:



The connectivity $\mu_{P \backslash a}(\{b\})=2+2-3=1$, so $\mu_{P \backslash a}(\{b\}) \neq r_{P \backslash a}(\{b\})$ and so $P \backslash a$ is not compact.

However, contraction does preserve compactness.

Theorem 3.19. Suppose that $P=\left(E, r_{P}\right)$ is a compact polymatroid, then $P / A$ is a compact polymatroid for any $A \subseteq E$.

Proof. We want to show that $\mu_{P / A}(\{e\})=r_{P / A}(\{e\})$ for any $e \in E-A$. To do this recall Lemma 3.5, that is, if $e$ is compact in $P$, then $r_{P}(E)=r_{P}(E-\{e\})$.

$$
\begin{aligned}
\mu_{P / A}(\{e\}) & =r_{P / A}(\{e\})+r_{P / A}((E-A)-\{e\})-r_{P / A}(E-A) \\
& =r_{P}(A \cup\{e\})-r_{P}(A)+r_{P}(E-\{e\})-r_{P}(A)-\left(r_{P}(E)-r_{P}(A)\right) \\
& =r_{P}(A \cup\{e\})-r_{P}(A) \\
& =r_{P / A}(\{e\}),
\end{aligned}
$$

where (1) follows from simplifying the line above and noticing that, as $P$ is compact, $r_{P}(E-\{e\})=r_{P}(E)$.

Our notion of duality does not interchange deletion and contraction, however the two operations do have a nice relation under duality. 
Theorem 3.20. If $P=(E, r)$ is a polymatroid, then $(P / A)^{*}=b\left(P^{*} \backslash A\right)$

Proof. First note that both $(P / A)^{*}$ and $b\left(P^{*} \backslash A\right)$ are defined on the same set, that is $E-A$. Consider $r_{b\left(P^{*} \backslash A\right)}(X)$ for $X \subseteq E-A$. We have the following chain of equalities:

$$
\begin{aligned}
r_{b\left(P^{*} \backslash A\right)}(X)= & \left(r_{P^{*} \backslash A}\right)(X)+\sum_{a \in X}\left[\left(\mu_{P^{*} \backslash A}\right)(\{a\})-\left(r_{P^{*} \backslash A}\right)(\{a\})\right] \\
= & \left(r_{P^{*} \backslash A}\right)(X)+\sum_{a \in X}\left[\left(r_{P^{*} \backslash A}\right)((E-A)-\{a\})-\left(r_{P^{*} \backslash A}\right)(E-A)\right] \\
= & r_{P^{*}}(X)+\sum_{a \in X}\left[r_{P^{*}}((E-A)-\{a\})-r_{P^{*}}(E-A)\right] \\
= & r_{P}(E-X)+\|X\|_{r_{P}}-r_{P}(E)+\sum_{a \in X}\left[\left(r_{P}(A \cup\{a\})\right.\right. \\
& \quad+\|E-(A \cup\{a\})\|_{r_{P}}-r_{P}(E)-\left(r_{P}(A)+\|E-A\|_{r_{P}}\right. \\
& \left.\left.\left.\quad-r_{P}(E)\right)\right)\right] \\
= & r_{P}(E-X)+\|X\|_{r_{P}}-r_{P}(E)+\sum_{a \in X}\left[\left(r_{P}(A \cup\{a\})+\|E\|_{r_{P}}\right.\right. \\
& \quad-\|A\| r_{P}-r_{P}(\{a\})-r_{P}(E)-r_{P}(A)-\|E\| r_{P}+\|A\|_{r_{P}} \\
& \left.\left.+r_{P}(E)\right)\right] \\
= & r_{P}(E-X)+\|X\|_{r_{P}}-r_{P}(E)+\sum_{a \in X}\left[r_{P}(A \cup\{a\})-r_{P}(\{a\})\right. \\
& \left.\quad-r_{P}(A)\right] \\
= & r_{P}(E-X)-r_{P}(E)+\sum_{a \in X}\left[r_{P}(A \cup\{a\})-r_{P}(A)\right] .
\end{aligned}
$$

Now consider $r_{(P / A)^{*}}(X)$ and consider the following chain of equalities:

$$
\begin{aligned}
r_{(P / A)^{*}}(X) & =\left(r_{P / A}\right)((E-A)-X)+\|X\|_{r_{P / A}}-\left(r_{P / A}\right)(E-A) \\
& =r_{P}(E-X)-r_{P}(A)+\|X\|_{r_{P / A}}-r_{P}(E)+r_{P}(A) \\
& =r_{P}(E-X)-r_{P}(E)+\sum_{a \in X}\left(r_{P / A}\right)(\{a\}) \\
& =r_{P}(E-X)-r_{P}(E)+\sum_{a \in X}\left[r_{P}(A \cup\{a\})-r_{P}(A)\right] .
\end{aligned}
$$

Therefore $(P / A)^{*}=b\left(P^{*} \backslash A\right)$

Earlier we proved that every connectivity function is the connectivity func- 
tion of a half-integral polymatroid. In a connectivity function we may remove elements via the elision operation. We now look at a corresponding operation for polymatroids.

In [8] it is proved that if we have a 2-polymatroid $P=\left(E, r_{P}\right)$ such that $a \in E$ and if we define $P \downarrow_{a}^{1}$ as a pair $\left(E-\{a\}, r_{P \downarrow_{a}^{1}}\right)$ where $r_{P \downarrow_{a}^{1}}$ is a set function from $E-\{a\}$ as defined as follows:

$$
r_{P \downarrow a}^{1}(X)= \begin{cases}r_{P}(X)-1 & \text { if } r_{P}(X)=r_{P}(X \cup\{a\}) \\ r_{P}(X) & \text { if } r_{P}(X) \neq r_{P}(X \cup\{a\}),\end{cases}
$$

then $P_{\lambda} \downarrow_{a}^{1}=P_{\lambda \downarrow a}$ where $P_{\lambda}$ is the connectivity polymatroid on $E$ generated by the unitary connectivity function $\lambda$. Intuitively this operation can be thought of as adding a point freely on $a$, contracting the added point and then deleting $a$. This motivates our next definition.

Definition 3.21. Suppose we have a polymatroid $P=\left(E, r_{P}\right)$ and an element $a \in E$ such that $r_{P}(\{a\})=k$. For $0 \leq l \leq k$ we define an $l$-squidge of a, denoted $P \downarrow_{a}^{l}$, by

$$
r_{P \downarrow_{a}}(X)=\min \left\{r_{P}(X), r_{P}(X \cup\{a\})-l\right\}
$$

Notice that deletion is just a 0 -squidge and contraction of a rank- $k$ element of $E$ is a $k$-squidge.

Intuitively an $l$-squidge of $a$ corresponds to putting $l$ points freely onto $a$, contracting them, then deleting $a$ (which will have dropped in rank by $l$ ). In practice, squidging will often be followed by compactification.

Lemma 3.22. Let $P=\left(E, r_{P}\right)$ be a polymatroid, and let $a \in E$ be such that $r_{P}(\{a\}) \geq l$ for some $l \in \mathbb{Z}$. Then the $l$-squidge of a in $P$ is a polymatroid.

Proof. First $r_{P \downarrow_{a}^{l}}(\emptyset)=\min \left\{r_{P}(\emptyset), r_{P}(\{a\})-l\right\}=0$ so $r_{P \downarrow_{a}^{l}}$ is normalised.

Next we show that $r_{P \downarrow_{a}^{l}}$ is increasing. To do this let $A \subseteq B \subseteq E-\{a\}$. We shall show that $\min \left\{r_{P}(A), r_{P}(A \cup\{a\})-l\right\} \leq \min \left\{r_{P}(B), r_{P}(B \cup\{a\})-l\right\}$.

Suppose $\min \left\{r_{P}(A), r_{P}(A \cup\{a\})-l\right\}=r_{P}(A)$ and $\min \left\{r_{P}(B), r_{P}(B \cup\right.$ $\{a\})-l\}=r_{P}(B)$. Then, as $r$ is increasing $r_{P}(A) \leq r_{P}(B)$, and so $\min \left\{r_{P}(A), r_{P}(A \cup\{a\})-l\right\} \leq \min \left\{r_{P}(B), r_{P}(B \cup\{a\})-l\right\}$. 
Suppose $\min \left\{r_{P}(A), r_{P}(A \cup\{a\})-l\right\}=r_{P}(A)$ and $\min \left\{r_{P}(B), r_{P}(B \cup\{a\})-\right.$ $l\}=r_{P}(B \cup\{a\})-l$. Then $r_{P}(A) \leq r_{P}(A \cup\{a\})-l \leq r_{P}(B \cup\{a\})-l$. Again this gives $\min \left\{r_{P}(A), r_{P}(A \cup\{a\})-l\right\} \leq \min \left\{r_{P}(B), r_{P}(B \cup\{a\})-l\right\}$. Suppose $\min \left\{r_{P}(A), r_{P}(A \cup\{a\})-l\right\}=r_{P}(A \cup\{a\})-l$ and $\min \left\{r_{P}(B), r_{P}(B \cup\right.$ $\{a\})-l\}=r_{P}(B \cup\{a\})-l$. Then $r_{P}(A \cup\{a\})-l \leq r_{P}(B \cup\{a\})-l$ as $r$ is increasing. This gives $\min \left\{r_{P}(A), r_{P}(A \cup\{a\})-l\right\} \leq \min \left\{r_{P}(B), r_{P}(B \cup\right.$ $\{a\})-l\}$.

Suppose $\min \left\{r_{P}(A), r_{P}(A \cup\{a\})-l\right\}=r_{P}(A \cup\{a\})-l$ and $\min \left\{r_{P}(B), r_{P}(B \cup\right.$ $\{a\})-l\}=r_{P}(B)$. Then $r_{P}(A \cup\{a\})-l \leq r_{P}(A) \leq r_{P}(B)$. This is the final case and gives $\min \left\{r_{P}(A), r_{P}(A \cup\{a\})-l\right\} \leq \min \left\{r_{P}(B), r_{P}(B \cup\{a\})-l\right\}$.

Therefore $r_{P \downarrow_{a}^{l}}(A) \leq r_{P \downarrow_{a}^{l}}(B)$ for all $A \subseteq B \subseteq E-\{a\}$.

Finally we must show that submodularity holds. To do this we take $A, B \subseteq$ $E-\{a\}$ and again split into cases as above.

If $r_{P \downarrow_{a}^{l}}(A)=r_{P}(A)$ and $r_{P \downarrow_{a}^{l}}(B)=r_{P}(B)$, then:

$$
\begin{aligned}
r_{P \downarrow_{a}^{l}}(A)+r_{P \downarrow_{a}^{l}}(B) & =r_{P}(A)+r_{P}(B) \\
& \geq r_{P}(A \cup B)+r_{P}(A \cap B) \\
& \geq r_{P \downarrow_{a}^{l}}(A \cup B)+r_{P \downarrow_{a}^{l}}(A \cap B),
\end{aligned}
$$

and so we are done.

If $r_{P \downarrow_{a}^{l}}(A)=r_{P}(A \cup\{a\})-l$ and $r_{P \downarrow_{a}}(B)=r_{P}(B)$, then:

$$
\begin{aligned}
r_{P \downarrow_{a}}(A)+r_{P \downarrow_{a}^{l}}(B) & =r_{P}(A \cup\{a\})-l+r_{P}(B) \\
& \geq r_{P}(A \cup\{a\} \cup B)-l+r_{P}((A \cup\{a\}) \cap B) \\
& =r_{P}(A \cup\{a\} \cup B)-l+r_{P}(A \cap B) \\
& \geq r_{P \downarrow_{a}^{l}}(A \cup B)+r_{P \downarrow_{a}^{l}}(A \cap B),
\end{aligned}
$$

and so we are done.

The proof of of the case where $r_{P \downarrow_{a}^{l}}(A)=r_{P}(A)$ and $r_{P \downarrow_{a}^{l}}(B)=r_{P}(B \cup\{a\})-l$ is the same as above. 
If $r_{P \downarrow_{a}^{l}}(A)=r_{P}(A \cup\{a\})-l$ and $r_{P \downarrow_{a}^{l}}(B)=r_{P}(B \cup\{a\})-l$, then:

$$
\begin{aligned}
r_{P \downarrow_{a}^{l}}(A)+r_{P \downarrow_{a}^{l}}(B) & =r_{P}(A \cup\{a\})-l+r_{P}(B \cup\{a\})-l \\
& \geq r_{P}(A \cup\{a\} \cup B)-l+r_{P}((A \cap B) \cup\{a\})-l \\
& \geq r_{P \downarrow_{a}^{l}}(A \cup B)+r_{P \downarrow_{a}^{l}}(A \cap B),
\end{aligned}
$$

and so we are done.

Therefore an $l$-squidge of an element $a$ of $P$ is a polymatroid.

Another way that squidging naturally arises in polymatroids is when the polymatroid is graphic. Then squidging an element, $e$, in the polymatroid corresponds to contracting edge $e$ in the graph. To see this, suppose that $G$ is a graph and let $P_{G}=\left(E(G), r_{G}\right)$ where $r_{G}(X)=\left|V_{G}(X)\right|$, where $V_{G}(X)$ is the set of vertices incident with some edge of $X$. Let $e \in E(G)$ and consider $P_{G} \downarrow_{e}^{1}$ which has rank function $r_{G} \downarrow_{e}^{1}(X)=\min \{r(X), r(X \cup\{e\})-1\}=$ $\min \{|V(X)|, \mid V(X \cup\{e\})-1\}$. In other words:

$$
r_{G} \downarrow_{e}^{1}(X)= \begin{cases}|V(X)|-1 & \text { if } V(X)=V(X \cup\{e\}) \\ |V(X)| & \text { if } V(X) \neq V(X \cup\{e\}) .\end{cases}
$$

Now consider $G / e$ and let $X \subseteq E(G)-\{e\}$. If $V_{G}(\{e\}) \subseteq V_{G}(X)$, then $\left|V_{G / e}(X)\right|=\left|V_{G}(X)\right|-1$. If $V_{G}(\{e\}) \nsubseteq V_{G}(X)$, then $\left|V_{G}(X)\right|=\left|V_{G / e}(X)\right|$. Now consider $P_{G / e}=\left(E(G / e), r_{G / e}\right)$ where $r_{G / e}=\left|V_{G / e}(X)\right|$; by the equalities above this means that:

$$
r_{G / e}(X)= \begin{cases}|V(X)|-1 & \text { if } V(X)=V(X \cup\{e\}) \\ |V(X)| & \text { if } V(X) \neq V(X \cup\{e\}),\end{cases}
$$

which is equal to $r_{G} \downarrow_{e}^{1}(X)$ for all $X \subseteq E(G)-\{e\}$. This means that we have proved the following lemma:

Lemma 3.23. Let $G$ and $P_{G}=\left(E(G), r_{G}\right)$ where $r_{G}(X)=|V(X)|$ for all $X \subseteq E(G)$. Then $P_{G} \downarrow_{e}^{1}=P_{G / e}$ for all $e \in E(G)$.

We now look at the behaviour of squidging under duality,

Lemma 3.24. Let $P=\left(E, r_{P}\right)$ be a polymatroid and a be a compact element of $E$ such that $r_{P}(\{a\})=k$. Then $\left(P \downarrow_{a}^{k-l}\right)^{*}=b\left(\left(P^{*}\right) \downarrow_{a}^{l}\right)$. 
Proof. We first prove the following claim:

Claim 3.24.1. $\left(r_{P^{*} \downarrow_{a}^{l}}\right)(X)=\|X\|_{r_{P}}-r_{P}(E)+(k-l)+\left(r_{P \downarrow_{a}^{k-l}}\right)((E-$ $\{a\})-X)$.

Proof. Recall that the rank function of the dual polymatroid is defined by $r_{P^{*}}(X)=r_{P}(E-X)+\|X\|_{r_{P}}-r_{P}(E)$, and that $r_{P}(\{a\})=k$.

$$
\begin{aligned}
\left(r_{P^{*} \downarrow_{a}^{l}}\right)(X)= & \min \left\{r_{P^{*}}(X), r_{P^{*}}(X \cup\{a\})-l\right\} \\
= & \min \left\{r_{P}(E-X)+\|X\|_{r_{P}}-r_{P}(E),\right. \\
& \left.\quad r_{P}(E-(X \cup\{a\}))+\|X\|_{r_{P}}+r_{P}(\{a\})-r_{P}(E)-l\right\} \\
= & \|X\|_{r_{P}}-r_{P}(E)+(k-l)+ \\
& \quad \min \left\{r_{P}(E-X)-(k-l), r_{P}((E-\{a\})-X)\right\} \\
= & \|X\|_{r_{P}}-r_{P}(E)+(k-l)+\left(r_{P_{\downarrow_{a}}^{k-l}}\right)((E-\{a\})-X) .
\end{aligned}
$$

Therefore, for any $x \in E-\{a\}$,

$r_{P^{*} \downarrow_{a}^{l}}((E-\{a\})-x)=\|E-\{a\} \mid\|_{r_{P}}-r_{P}(\{x\})-r_{P}(E)+(k-l)+r_{P \downarrow_{a}^{k-l}}(\{x\})$,

and

$$
r_{P^{*} \downarrow_{a}^{l}}(E-\{a\})=\|E-\{a\}\|_{r_{P}}-r_{P}(E)+(k-l) .
$$

By the definition of duality

$$
r_{\left(P \downarrow_{a}^{k-l}\right)^{*}}(X)=r_{P \downarrow_{a}^{k-l}}((E-\{a\})-X)+\|X\|_{r_{P \downarrow a}^{k-l}}-r_{P \downarrow_{a}^{k-l}}(E-\{a\}),
$$

which, by the defintion of squidging and as $a$ is compact in $P$, is equal to

$$
r_{\left(P \downarrow_{a}^{k-l}\right)^{*}}(X)=r_{P \downarrow_{a}^{k-l}}((E-\{a\})-X)+\|X\|_{r_{P \downarrow a}^{k-l}}-r_{P}(E)+(k-l)
$$


We now consider the compactification of $r_{P^{*} \downarrow a}^{l}$.

$$
\begin{aligned}
&\left(r_{b\left(P^{*} \downarrow_{a}^{l}\right)}\right)(X)= r_{P^{*} \downarrow_{a}^{l}}(X)+\sum_{x \in X}\left[\mu_{P^{*} \downarrow_{a}^{l}}(\{x\})-r_{P^{*} \downarrow_{a}^{l}}(\{x\})\right] \\
&= r_{P \downarrow_{a}^{k-l}}((E-\{a\})-X)+(k-l)-r_{P}(E)+ \\
&\|X\|_{r_{P}}+\sum_{x \in X}\left[\mu_{P^{*} \downarrow_{a}^{l}}(\{x\})-r_{P^{*} \downarrow_{a}^{l}}(\{x\})\right] \\
&= r_{P \downarrow_{a}^{k-l}}((E-\{a\})-X)+(k-l)-r_{P}(E)+ \\
& \sum_{x \in X}\left[\mu_{P^{*} \downarrow_{a}^{l}}(\{x\})-r_{P^{*} \downarrow_{a}^{l}}(\{x\})+r_{P}(\{x\})\right] \\
&= r_{P \downarrow_{a}^{k-l}}((E-\{a\})-X)+(k-l)-r_{P}(E)+ \\
& \sum_{x \in X}\left[r_{P^{*} \downarrow_{a}^{l}}((E-\{a\})-\{x\})-r_{P^{*} \downarrow_{a}^{l}}(E-\{a\})+r_{P}(\{x\})\right] \\
&= r_{P \downarrow_{a}^{k-l}}((E-\{a\})-X)+(k-l)-r_{P}(E)+ \\
& \sum_{x \in X}\left[\left.\|E-\{a\}\|\right|_{r_{P}}-r_{P}(\{x\})-r_{P}(E)+(k-l)+r_{P \downarrow_{a}^{k-l}}(\{x\})\right. \\
&\left.-\left(\|E-\{a\}\|_{r_{P}}-r_{P}(E)+(k-l)\right)+r_{P}(\{x\})\right] \\
&= r_{P \downarrow_{a}^{k-l}}((E-\{a\})-X)+(k-l)-r_{P}(E)+\sum_{x \in X} r_{P \downarrow_{a}^{k-l}}(\{x\}) \\
&= r_{P \downarrow_{a}^{k-l}}((E-\{a\})-X)+(k-l)-r_{P}(E)+\|X\|_{r_{\downarrow_{a} k-l}^{k-l}} \\
&=
\end{aligned}
$$

where (2) follows from (1) by the claim, (4) follows from (3) by the definition of $\mu_{P^{*} \downarrow_{a}^{l}}(\{x\})$, (5) follows from (4) by (i) and (ii), and (8) follows from (7) by (iii).

Recall that a polymatroid is called connected if $r_{P}(X)+r_{P}(E-X)>r_{P}(E)$ for all non-empty $X \subsetneq E$. Tutte proved in [13] that if a matroid $M=(E, r)$ is connected then, for any element $a$ in $E$, either $M \backslash a$ or $M / a$ is connected. This is not in general true for connected polymatroids. For consider the polymatroid, $P$, given below: 


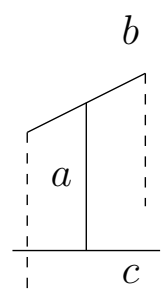

The rank function is as follows: $r_{P}(\{a\})=2, r_{P}(\{b\})=2, r_{P}(\{c\})=$ $2, r_{P}(\{a, b\})=3, r_{P}(\{a, c\})=3, r_{P}(\{b, c\})=4, r_{P}(\{a, b, c\})=4$.

The rank function of $P \backslash a$ is: $r_{P \backslash a}(\{b\})=2, r_{P \backslash a}(\{c\})=2, r_{P \backslash a}(\{b, c\})=4$, and this is not connected as $r_{P \backslash a}(\{b\})+r_{P \backslash a}(\{c\})=r_{P \backslash a}(\{b, c\})$.

The rank function of $P / a$ is: $r_{P \backslash a}(\{b\})=1, r_{P \backslash a}(\{c\})=1, r_{P \backslash a}(\{b, c\})=2$, and this is not connected as $r_{P \backslash a}(\{b\})+r_{P \backslash a}(\{c\})=r_{P \backslash a}(\{b, c\})$

We also have the following example of a 2-polymatroid, $P$, that is connected but has an element $a$ such that $P \downarrow_{a}^{1}$ is not connected. However, note that $P / a$ is connected.

$r_{P}(\{a\})=2, r_{P}(\{b\})=2, r_{P}(\{c\})=2, r_{P}(\{a, b\})=4, r_{P}(\{a, c\})=$ $4, r_{P}(\{b, c\})=4, r_{P}(\{a, b, c\})=5$.

Consider $P \downarrow_{a}^{1}$, whose rank function is: $r_{P}(\{b\})=2, r_{P}(\{c\})=2, r_{P}(\{b, c\})=$ 4. This is clearly not connected.

Consider $P / a$, whose rank function is: $r_{P}(\{b\})=2, r_{P}(\{c\})=2, r_{P}(\{b, c\})=$ 3. This is connected.

Also note that, in this example, $a$ is not compact as $r_{P}(E) \neq r_{P}(E-\{a\})$.

We shall shortly prove that if $P$ is a connected polymatroid, then, for any $a \in E$ there is some $0 \leq j \leq r_{P}(a)$ such that $P \downarrow_{a}^{j}$ is a connected polymatroid. To do this we introduce an operation known as principal truncation, which is very closely related to squidging.

Intuitively principal truncation is adding a point freely on an element of $P$ and contracting that point. Performing principal truncation on a polymatroid results in a polymatroid, see [4].

Definition 3.25. Let $P=\left(E, r_{P}\right)$ be a polymatroid and let $a \in E$. We denote the principal truncation of $a$ by $T_{a}(P)=\left(E, r_{T_{a}(P)}\right)$ where $r_{T_{a}(P)}$ is 
defined as follows:

$$
r_{T_{a}(P)}(X)= \begin{cases}r_{P}(X)-1 & \text { if } r_{P}(X \cup\{a\})=r_{P}(X) \\ r_{P}(X) & \text { otherwise }\end{cases}
$$

Observe that a 1-squidge of an element $e$ of $P$ is just the principal truncation of $e$ in $P$ followed by the deletion of $e$. An $l$-squidge for $l<r_{P}(\{e\})$ is just the principal truncation of $e$ in $P$ followed by an $(l-1)$-squidge of $e$ in $P$. This is an important observation and will be used in inductive arguments later on.

In the proof that if $P$ is connected then $P \downarrow_{a}^{j}$ is connected for some $j$, we shall use the following lemma, which is proved in [4]

Lemma 3.26. Let $P=\left(E, r_{P}\right)$ be a connected $k$-polymatroid with $e \in E$. Then $T_{e}(P)$ is connected if, and only if, $P$ is connected and $r_{P}(\{e\})>1$.

We also need to introduce the notion of closure in a polymatroid. This is very similar to closure in a matroid.

Definition 3.27. Let $P=(E, r)$ be a polymatroid and let $X \subset E$. The closure of $X$, denoted $\operatorname{cl}(X)$, is equal to $\{x \in E: r(X \cup\{x\})=r(X)\}$.

The proof of the following lemma is simple.

Lemma 3.28. Let $P=(E, r)$ be a polymatroid and suppose that $X \subseteq E$ Then the following hold:

$$
\begin{aligned}
& \text { 1. } r(\operatorname{cl}(X))=r(X) \\
& \text { 2. } \operatorname{cl}(\operatorname{cl}(X))=\operatorname{cl}(X) \\
& \text { 3. If } X \subseteq Y \subseteq E \text { then } \operatorname{cl}(X) \subseteq \operatorname{cl}(Y)
\end{aligned}
$$

This ensures that the closure of a polymatroid is indeed a closure operator.

Lemma 3.29. Suppose that $P$ is a connected polymatroid, and that $P \backslash a$ is not connected, where $a$ is a rank-1 element of $E$. Let $\emptyset \neq X \neq E$ be such that $\mu_{P \backslash a}(X)=0$. Then $a \notin \operatorname{cl}(X)$ and $a \notin \operatorname{cl}((E-\{a\})-X)$. 
Proof. As

$$
\begin{aligned}
\mu_{P \backslash a}(X) & =0 \\
& =r_{P \backslash a}(X)+r_{P \backslash a}((E-\{a\})-X)-r_{P \backslash a}(E-\{a\}) \\
& =r_{P \backslash a}(X)+r_{P \backslash a}((E-\{a\})-X)-r_{P}(E),
\end{aligned}
$$

then $\mu_{P}(X)=r_{P}(X)+r_{P}(E-X)-r_{P}(E) \leq 1$. As $P$ is connected this means that $\mu_{P}(X)=1$. A similar argument shows that $\mu_{P}(X \cup\{a\})=1$.

Suppose that $r_{P}(E-X)=r_{P}((E-\{a\})-X)$, that is, $a \in \operatorname{cl}((E-\{a\})-X)$. Then:

$$
\begin{aligned}
0 & =\mu_{P \backslash a}(X) \\
& =r_{P \backslash a}(X)+r_{P \backslash a}((E-\{a\})-X)-r_{P \backslash a}(E-\{a\}) \\
& =r_{P}(X)+r_{P}((E-\{a\})-X)-r_{P}(E-\{a\}) \\
& =r_{P}(X)+r_{P}(E-X)-r_{P}(E) \\
& =\mu_{P}(X),
\end{aligned}
$$

a contradiction to the fact that $\mu$ is connected. Therefore $a \notin \operatorname{cl}((E-$ $\{a\})-X)$. As $r_{P}(X)+r_{P}(E-X)-r_{P}(E)=1=r_{P}(X \cup\{a\})+r_{P}(E-$ $(X \cup\{a\}))-r_{P}(E)$, and $r_{P}\left(E-(X \cup\{a\})=r_{P}(E-X)-1\right.$, we must also have $r_{P}(X \cup\{a\})=r_{P}(X)+1$. This means that $a \notin \operatorname{cl}(X)$ and $a \notin$ $\operatorname{cl}((E-\{a\})-X)$.

We also need to recall, from Section 2.3, that a direct sum of two connectivity functions, $\lambda_{1}$ and $\lambda_{2}$, on sets $E_{1}$ and $E_{2}$ respectively, is defined by $\lambda_{1} \oplus$ $\lambda_{2}(X)=\lambda_{1}\left(X \cap E_{1}\right)+\lambda_{2}\left(X \cap E_{2}\right)$. Also recall Lemma 2.16. That is, if $\lambda$ is a connectivity function on a set $E$, such that some non-empty, proper subset, $X$, of $E$ is such that $\lambda(X)=0$, then there are connectivity functions $\lambda_{1}$ on $X$ and $\lambda_{2}$ on $E-X$ such that $\lambda=\lambda_{1} \oplus \lambda_{2}$.

Theorem 3.30. Let $P$ be a connected polymatroid, then, for any $a \in E$, there is some $0 \leq j \leq r(\{a\})$ such that $P \downarrow_{a}^{j}$ is connected.

Proof. The proof is by induction on $r(\{a\})$ and the following claim provides the base case: 
Claim 3.30.1. If $r(\{a\})=1$ then either $P \backslash a$ or $P / a$ is connected.

Proof. Suppose that $P \backslash a$ were not connected, and let $\mathcal{S}$ be the collection of all minimal separators (that is, connected components) of $P \backslash a$. Let $\emptyset \neq Y \subsetneq E-\{a\}$.

First suppose that there is some $S \in \mathcal{S}$ such that $Y \cap S=\emptyset$. As $S \in \mathcal{S}$ we have $\lambda_{P \backslash a}(S)=0$, and as $P$ is connected, by Lemma 3.29, this means that $a \notin \operatorname{cl}(S)$ and $a \notin \operatorname{cl}((E-\{a\})-S)$. As $Y \cap S=\emptyset$, this means that $Y \subseteq(E-\{a\})-S$, and so $a \notin \operatorname{cl}(Y)$. Therefore:

$$
\begin{aligned}
\lambda_{P / a}(Y) & =r_{P / a}(Y)+r_{P / a}((E-\{a\})-Y)-r_{P / a}(E-\{a\}) \\
& =r_{P}(Y \cup\{a\})-r_{P}(\{a\})+r_{P}(E-Y)-r_{P}(\{a\})-r_{p}(E)+r_{P}(\{a\}) \\
& =r_{P}(Y \cup\{a\})+r_{P}(E-Y)-r_{p}(E)-1 \\
& =r_{P}(Y)+r_{P}(E-Y)-r_{P}(E) \\
& =\lambda_{P}(Y) \\
& >0
\end{aligned}
$$

where (1) follows as $r_{P}(\{a\})=1$, (2) follows as $a \notin \operatorname{cl}(Y)$, so $r_{P}(Y \cup\{a\})=$ $r_{P}(Y)+1$, and (3) follows as $P$ is connected. Therefore if $Y \cap S=\emptyset$ for some $S \in \mathcal{S}$, then $\lambda_{P / a}(Y)>0$.

Next suppose that there is no $S \in \mathcal{S}$ such that $Y \cap S=\emptyset$. Further suppose that $Y \cap S_{i}=S_{i}$ for all $S_{i} \in \mathcal{S}-S$ for some $S \in \mathcal{S}$. If $Y \cap S_{i}=S_{i}$ for all $S_{i} \in \mathcal{S}$, then $Y=E-\{a\}$, a contradiction. Let $S_{1}$ be a member of $\mathcal{S}$ that is such that $Y \cap S_{1} \neq S_{1}$. As there is only one such member, this means that $(E-\{a\})-S_{1} \subseteq Y$, and so $(E-\{a\})-Y \subseteq S_{1}$. Therefore $a \notin \operatorname{cl}((E-\{a\})-Y)$. Therefore

$$
\begin{aligned}
\lambda_{P / a}(Y) & =r_{P / a}(Y)+r_{P / a}((E-\{a\})-Y)-r_{P / a}(E-\{a\}) \\
& =r_{P}(Y \cup\{a\})-r_{P}(\{a\})+r_{P}(E-Y)-r_{P}(\{a\})-r_{p}(E)+r_{P}(\{a\}) \\
& =r_{P}(Y \cup\{a\})+r_{P}(E-Y)-r_{p}(E)-1 \\
& =r_{P}(Y)+r_{P}(E-Y)-r_{P}(E) \\
& =\lambda_{P}(Y) \\
& >0
\end{aligned}
$$


and so, if there is only one member of $\mathcal{S}$ that is not fully contained in $Y$, then $\lambda_{P / a}(Y)>0$.

Finally suppose that $Y$ has a non-zero intersection with every member of $\mathcal{S}$, and further suppose that at least two members of $\mathcal{S}$ are not fully contained in $Y$. Let these members of $\mathcal{S}$ be $S_{1}$ and $S_{2}$. By Lemma 2.16 and the definition of a direct sum of connectivity functions, $\lambda_{P \backslash a}(Y)=$ $\lambda_{P \backslash a}\left(S_{1} \cap Y\right)+\lambda_{P \backslash a}\left(S_{2} \cap Y\right)+\cdots+\lambda_{P \backslash a}\left(S_{n} \cap Y\right)$ where $\mathcal{S}=\left\{S_{1}, \ldots, S_{n}\right\}$. As $S_{i} \cap Y$ is non-empty and is not equal to $S_{i}$, for $i \in\{1,2\}$, this means that $\lambda_{P \backslash a}\left(S_{1} \cap Y\right)+\lambda_{P \backslash a}\left(S_{2} \cap Y\right) \geq 2$, and so $\lambda_{P \backslash a}(Y) \geq 2$. This means that $\lambda_{P}(Y) \geq 2$ as:

$$
\begin{aligned}
\lambda_{P \backslash a}(Y) & =r_{P}(Y)+r_{P}((E-\{a\})-Y)-r_{P}(E-\{a\}) \\
& =r_{P}(Y)+r_{P}((E-\{a\})-Y)-r_{P}(E) \\
& \leq r_{P}(Y)+r_{P}(E-Y)-r_{P}(E) \\
& =\lambda_{P}(Y),
\end{aligned}
$$

where (1) follows as $a$ is compact in $P$ and has rank 1 .

Now consider $\lambda_{P / a}(Y)$.

$$
\begin{aligned}
\lambda_{P / a}(Y) & =r_{P / a}(Y)-r_{P / a}((E-\{a\})-Y)-r_{P / a}(E-\{a\}) \\
& =r_{P}(Y \cup\{a\})-r_{P}(\{a\})+r_{P}(E-Y)-r_{P}(\{a\})-r_{P}(E)+r_{P}(\{a\}) \\
& =r_{P}(Y \cup\{a\})+r_{P}(E-Y)-r_{P}(E)-1 \\
& \geq r_{P}(Y)+r_{P}(E-Y)-r_{P}(E)-1 \\
& =\lambda_{P}(Y)-1 \\
& \geq 1 .
\end{aligned}
$$

Therefore, if $P \backslash a$ is not connected, then for every possible choice of $\emptyset \neq$ $Y \subsetneq E, \lambda_{P / a}(Y)>0$; that is $P / a$ is connected. This means that, for any $a \in E$, either $P \backslash a$ is connected or $P / a$ is connected.

Suppose for the purposes of induction that the result holds when $r_{P}(\{a\})=$ $k-1$, and let $r_{P}(\{a\})=k$.

As $P$ is connected $T_{a}(P)$ is connected and $r_{T_{a}(P)}(\{a\})=k-1$ so for some $0 \leq j \leq k-1$, by the induction hypothesis we have $\left(T_{a}(P)\right) \downarrow_{a}^{j}$ is connected. 
Therefore $P \downarrow_{a}^{j+1}$ is connected and $j+1 \leq k$ so we have found some squidge of $P$ that is connected.

Definition 3.31. Let $P=\left(E, r_{P}\right)$ be a polymatroid and let $e \in E$ such that $r_{P}(\{e\})=k$. An intermediate-squidge of $e$ is any squidge of $e$ in $P$ that is not a 0 -squidge or a $k$-squidge.

We shall now show that, when $a$ is a compact element of a connected polymatroid, $P$, any intermediate squidge of $a$ results in a connected polymatroid. We first show this for a rank-2 element of $P$ (in other words we show that $P \downarrow_{a}^{1}$ is connected) and then use this as a base case to prove the result when $r_{P}(\{a\})=k$.

Lemma 3.32. Suppose that $a$ is a non-compact element of $P=\left(E, r_{P}\right)$. Then for every $X \subseteq E-\{a\}$ we have $r_{P}(X)<r_{P}(X \cup\{a\})$.

Proof. Suppose that $r_{P}(X \cup\{a\})=r_{P}(X)$ for some $X \subseteq E-\{a\}$. Then $a \in \operatorname{cl}(X)$. This means that $a \in \operatorname{cl}(E-\{a\})$, as $X \subseteq E-\{a\}$, and therefore $r_{P}(E-\{a\})=r_{P}(E) ;$ a contradiction.

Lemma 3.33. Let $P=\left(E, r_{P}\right)$ be a polymatroid and let $a \in E$. Then

$$
r_{P \downarrow a}(X)= \begin{cases}r_{P}(X) & \text { if } a \notin \operatorname{cl}(X) \\ r_{P}(X)-1 & \text { if } a \in \operatorname{cl}(X)\end{cases}
$$

Proof. Suppose that $a \notin \operatorname{cl}(X)$; then $r_{P}(X \cup\{a\})>r_{P}(X)$. Therefore, $\min \left\{r_{P}(X), r_{P}(X \cup\{a\})-1\right\}=r_{P}(X)$. Suppose that $a \in \operatorname{cl}(X)$; then $r_{P}(X \cup\{a\})=r_{P}(X)$. Therefore, $\min \left\{r_{P}(X), r_{P}(X \cup\{a\})-1\right\}=r_{P}(X \cup$ $\{a\})-1=r_{P}(X)-1$.

Lemma 3.34. Let $P$ be a connected polymatroid and a be a compact element of $P$ such that $r(\{a\})=2$. Then $P \downarrow_{a}^{1}$ is a connected polymatroid.

Proof. Let $X \subseteq E-\{a\}$, and $Y=(E-\{a\})-X$. First suppose that $a \in \operatorname{cl}(X)$ and $a \in \operatorname{cl}(Y)$. Then, by Lemma 3.33, it is clear that $r_{P \downarrow \frac{1}{1}}(X)+$ $r_{P \downarrow_{a}^{1}}(Y)=r_{P}(X \cup\{a\})+r_{P}(Y \cup\{a\})-2$, and, as $a$ is compact, $r_{P \downarrow_{a}^{1}}(E-\{a\})=$ 
$r_{P}(E)-1$ Therefore:

$$
\begin{aligned}
r_{P \downarrow \frac{1}{a}}(X)+r_{P \downarrow \frac{1}{a}}(Y) & =r_{P}(X \cup\{a\})+r_{P}(Y \cup\{a\})-2 \\
& \geq r_{P}(X \cup Y \cup\{a\})+r_{P}(\{a\})-2 \\
& =r_{P}(E) \\
& >r_{P \downarrow_{a}}(E-\{a\}),
\end{aligned}
$$

where(1) follows by submodularity. Therefore $r_{P \downarrow_{a}^{1}}(X)+r_{P \downarrow_{a}^{1}}(Y)>r_{P \downarrow_{a}^{1}}(E-$ $\{a\})$.

Next suppose that $a \in \operatorname{cl}(X)$ and $a \notin \operatorname{cl}(Y)$. Then, by Lemma 3.33, we have $r_{P \downarrow \frac{1}{a}}(X)+r_{P \downarrow_{a}^{1}}(Y)=r_{P}(X \cup\{a\})+r_{P}(Y)-1$, and, as $a$ is compact, $r_{P \downarrow \frac{1}{a}}(E-\{a\})=r_{P}(E)-1$. Therefore:

$$
\begin{aligned}
r_{P \downarrow_{a}^{1}}(X)+r_{P \downarrow_{a}^{1}}(Y) & =r_{P}(X \cup\{a\})+r_{P}(Y)-1 \\
& >r_{P}(E)-1 \\
& =r_{P \downarrow_{a}^{1}}(E-\{a\}),
\end{aligned}
$$

where (2) follows from (1) as $(X \cup\{a\}, Y)$ is a partition of $E$, the set $Y$ is nonempty, and $P$ is connected. Therefore $r_{P \downarrow \frac{1}{a}}(X)+r_{P \downarrow a}(Y)>r_{P \downarrow \frac{1}{a}}(E-\{a\})$. The proof of the case where $a \notin \operatorname{cl}(X)$ and $a \in \operatorname{cl}(Y)$ is the same.

Finally, suppose that $a \notin \operatorname{cl}(X)$ and $a \notin \operatorname{cl}(Y)$. Then, by Lemma 3.33, we have $r_{P \downarrow \frac{1}{a}}(X)+r_{P \downarrow \frac{1}{a}}(Y)=r_{P}(X)+r_{P}(Y)$, and, as $a$ is compact, $r_{P \downarrow \frac{1}{a}}(E-$ $\{a\})=r_{P}(E)-1$. Therefore:

$$
\begin{aligned}
r_{P \downarrow a}^{1 \frac{1}{a}}(X)+r_{P \downarrow a}^{1}(Y) & =r_{P}(X)+r_{P}(Y) \\
& \geq r_{P}(X \cup Y) \\
& =r_{P}(E-\{a\}) \\
& =r_{P}(E) \\
& >r_{P \downarrow \frac{1}{a}}(E-\{a\}),
\end{aligned}
$$

where (4) follows from (3) by the compactness of $a$. Therefore $r_{P \downarrow \frac{1}{a}}(X)+$ $r_{P \downarrow_{a}^{1}}(Y)>r_{P \downarrow \frac{1}{a}}(E-\{a\})$. 
Before we prove that any intermediate squidge of any element of $P$ results in a connected polymatroid we take a brief detour and prove the following easy corollaries of Lemma 3.34. These theorems are both known, but the proofs become much shorter when we view the objects as polymatroids, and use squidging. This helps to provide motivation for the operation.

Corollary 3.35. Let $G$ be a connected graph, then $G / e$ is a connected graph for any $e \in E(G)$.

Proof. Consider the polymatroid, $P_{G}$ obtained from $G$. The polymatroid $P_{G} \downarrow_{e}^{1}$ is connected by Lemma 3.34 , and we showed earlier that $P_{G} \downarrow_{e}^{1}=P_{G / e}$. Therefore $P_{G / e}$ is connected so $G / e$ is connected.

Definition 3.36. Let $M$ be a matroid and $a, b$ be elements of $M$. Then $a$ and $b$ are clones in $M$ if there is an automorphism on $M$ that interchanges $a$ and $b$ and fixes all other elements. When $a$ and $b$ are clones in $M$ then we call $\{a, b\}$ a clonal pair in $M$.

Clones are used frequently in [3] and the following corollary also comes easily from Lemma 3.34 .

Corollary 3.37. Suppose $M$ is a connected matroid and $\{a, b\}$ is a clonal pair in $M$, then deleting one of $a, b$ and contracting the other will result in a connected matroid.

Proof. Consider the 2-polymatroid that has the same groundset and rank function as $M$ except that elements $a$ and $b$ of the groundset have been replaced by a single rank- 2 element $l$ with the property that $r_{P}(A \cup\{l\})=$ $r_{M}(A \cup\{a, b\})$ for all $A \subseteq E-\{a, b\}$.

Claim 3.37.1. The polymatroid resulting from squidging $l$ is the same as the matroid resulting from deleting $a$ and contracting $b$.

Proof. Consider $r_{M \backslash a / b}(A)$ and $r_{P \downarrow_{l}^{1}}(A)$ for any $A \subseteq E-\{a, b\}$. By the definition of deletion and contraction in a matroid, $r_{M \backslash a / b}(A)=r_{M}(A \cup$ $\{b\})-r_{M}(\{b\})$, and recall $r_{P \downarrow_{l}}(A)=\min \left\{r_{P}(A), r_{P}(A \cup\{l\})-1\right\}$. 
If $b \in \operatorname{cl}(A)$, then $r_{M \backslash a / b}(A)=r_{M}(A)-r_{M}(\{b\})$. As $b \in \operatorname{cl}(A)$ and $\{a, b\}$ is a clonal pair, $a \in \operatorname{cl}(A)$. Therefore $r_{P}(A \cup\{l\})=r_{M}(A \cup\{a, b\})=r_{M}(A)$. Therefore $r_{P_{\downarrow} 1}(A)=r_{P}(A \cup\{l\})-1=r_{P}(A)-1=r_{M}(A)-1=r_{M \backslash a / b}(A)$. If $b \notin \operatorname{cl}(A)$, then $r_{M \backslash a / b}(A)=r_{M}(A)$ (note that if $b \notin \operatorname{cl}(A)$, then $r(\{b\}) \neq$ $0)$. We also have $r_{P}(A \cup\{l\})>r_{P}(A)$ so $r_{P \downarrow_{l}}(A)=r_{M}(A)=r_{M \backslash a / b}(A)$.

By Lemma 3.34, $P \downarrow_{l}^{1}$ is connected, and $P \downarrow_{l}^{1}=M \backslash a / b$ so $M \backslash a / b$ is connected.

We now prove that any intermediate squidge of any element of $P$, a connected polymatoid, preserves connectedness. To do this we shall use the following lemma:

Lemma 3.38. Let e be a compact element of $P$, then e is a compact element of $T_{a}(P)$.

Proof. $\mu_{T_{a}(P)}(\{e\})=r_{T_{a}(P)}(\{e\})+r_{T_{a}(P)}(E-\{e\})-r_{T_{a}(P)}(E)$. If $e \neq a$ then $r(E-\{e\})=r((E-\{e\}) \cup\{a\})$ and if $e=a$ then, as $e$ is compact in $P$, we have $r(E-\{e\})=r(E)=r((E-\{e\}) \cup\{a\})$. Therefore $r_{T_{a}(P)}(E-\{e\})=$ $r(E-\{e\})-1$.

Therefore $\mu_{T_{a}(P)}(\{e\})=r_{T_{a}(P)}(\{e\})+r(E-\{e\})-1-(r(E)-1)=r_{T_{a}(P)}(\{e\})$ by compactness of $e$ in $P$; and so $e$ is compact in $T_{a}(P)$.

In other words, principal truncation preserves compactness.

Before stating the next theorem, we note that an intermediate squidge is only defined for elements of rank at least 2 .

Theorem 3.39. Let $P=(E, r)$ be a connected, compact polymatroid. Then any intermediate-squidge of an element, a, of $E$, in $P$, results in a connected polymatroid.

Proof. Let $r_{P}(\{a\})=k$. The result holds when $k=2$ by Lemma 3.34 . Suppose, for the purposes of induction, that the result holds for all $a$ such that $r_{P}(\{a\})<k$.

Let $r_{P}(\{a\})=k$ and consider $T_{a}(P)$. As $P$ is connected and compact and $r_{P}(\{a\})>1$, we have $T_{a}(P)$ is also connected and compact by Lemmas 3.26 and 3.38, and $r_{T_{a}(P)}(\{a\})=k-1$. Therefore $\left(T_{a}(P)\right) \downarrow_{a}^{j}$ is connected for 
all $1 \leq j \leq k-1$, and so $P \downarrow_{a}^{j+1}$ is connected. Therefore all intermediate squidges result in a connected polymatroid except, possibly, when $j=1$. We shall now prove that $P \downarrow_{a}^{1}$ is connected.

Consider $\left(P^{*} \downarrow_{a}^{k-1}\right)^{*}$. This is connected, as the dual of a connected polymatroid is connected and $P^{*} \downarrow_{a}^{k-1}$ is connected, as $r_{P}(a)=k$. By Lemma 3.24 . as $P$ is compact, we have $\left(P^{*} \downarrow_{a}^{k-1}\right)^{*}=b\left(P^{* *} \downarrow_{a}^{1}\right)=b\left(P \downarrow_{a}^{1}\right)$. Therefore, by Lemma 3.15, $P \downarrow_{a}^{1}$ is connected.

\section{$3.5 \quad 2$-sums and Unavoidable Squidge-Minors of 2-polymatroids}

Using squidging as a minor operation can sometimes simplify solutions quite drastically. We demonstrate this by looking at the following result from [4]. In [4], Hall gives the unavoidable minors for 2-polymatroids when we define a minor of a 2-polymatroid, $P$, as a polymatroid obtained from $P$ be a series of deletions and contractions. Recall that a squidge-minor of a polymatroid $P$, is a polymatroid obtained from $P$ be a series of deletions, contractions and squidges. When we look at the unavoidable squidge-minors of a connected 2-polymatroid rather than just the unavoidable minors, this list is greatly reduced.

First we introduce various ways of adding polymatroids.

Recall from the previous section that if we have two polymatroids, $P_{1}=$ $\left(E_{1}, r_{1}\right)$ and $P_{2}=\left(E_{2}, r_{2}\right)$, on disjoint groundsets, then $P_{1} \oplus P_{2}=\left(E_{1} \cup E_{2}, r\right)$ where $r$ is defined as follows:

$$
r(X)=r_{1}\left(X \cap E_{1}\right)+r_{2}\left(X \cap E_{2}\right)
$$

for all $X \subseteq E_{1} \cup E_{2}$, and this is known as the direct sum of $P_{1}$ and $P_{2}$.

Definition 3.40. Suppose we have two $k$-polymatroids $P_{1}=\left(E, r_{1}\right)$ and $P_{2}=\left(E, r_{2}\right)$ on the same groundset, then we call $P_{1}+P_{2}$ the general sum of $P_{1}$ and $P_{2}$ and define $r_{P_{1}+P_{2}}$ as follows:

$$
r_{P_{1}+P_{2}}(X)=r_{1}(X)+r_{2}(X)
$$


for all $X \subseteq E$.

Definition 3.41. Let $A=\left(E_{1}, r_{1}\right)$ and $B=\left(E_{2}, r_{2}\right)$ be $k$-polymatroids with $E_{1} \cap E_{2}=\{p\}$ and $r_{1}(\{p\})=r_{2}(\{p\})$. Then we define a pair $A \oplus_{2} B=$ $\left(\left(E_{1} \cup E_{2}\right), r_{M_{1} \oplus_{2} M_{2}}\right)$ where we define $r_{M_{1} \oplus_{2} M_{2}}$ as follows:

$$
r_{M_{1} \oplus_{2} M_{2}}(A)=\min \left\{r_{1}\left(A_{1}\right)+r_{2}\left(A_{2}\right), r_{1}\left(A_{1} \cup p\right)+r_{2}\left(A_{2} \cup p\right)-r_{1}(p)\right\}
$$

where $A \subseteq\left(E_{1} \cup E_{2}\right)-p$ and $A_{1}=A \cap E_{1}$ and $A_{2}=A \cap E_{2}$.

We call $A \oplus_{2} B$ the 2-sum of $A$ and $B$.

It can easily be seen that the direct sum, the general sum, and 2-sum of two polymatroids are polymatroids.

Theorem 3.42. If $P_{1}=\left(E_{1}, r_{1}\right)$ and $P_{2}=\left(E_{2}, r_{2}\right)$ are polymatroids, $E_{1} \cap$ $E_{2}=p$ and $r_{1}(p)=r_{2}(p)$, then $P_{1} \downarrow_{e}^{l} \oplus_{2} P_{2}=\left(P_{1} \oplus_{2} P_{2}\right) \downarrow_{e}^{l}$, for any $e \in E_{1}-\{p\}$

Proof. First note that

$\min \left\{\min \left\{P_{1}, P_{2}\right\}, \min \{C, D\}\right\}=\min \left\{\min \left\{P_{1}, C\right\}, \min \left\{P_{2}, D\right\}\right\}$.

Let $e \in P_{1}$ and $e \neq p$ and consider $r_{\left(P_{1} \oplus_{2} P_{2}\right) \downarrow_{e}^{l}}$. For all $X \in\left(E_{1} \cup E_{2}\right)-p$ we let $X_{1}=E_{1} \cap X$ and $X_{2}=E_{2} \cap X$ and get the following chain of equalities:

$$
\begin{aligned}
r_{\left(P_{1} \oplus_{2} P_{2}\right) \downarrow_{e}^{l}}(X)= & \min \left\{r_{P_{1} \oplus_{2} P_{2}}(X), r_{P_{1} \oplus P_{2}}(X \cup\{e\})-l\right\} \\
= & \min \left\{\min \left\{r_{1}\left(X_{1}\right)+r_{2}\left(X_{2}\right), r_{1}\left(X_{1} \cup p\right)+r_{2}\left(X_{2} \cup p\right)-r_{1}(p)\right\},\right. \\
& \min \left\{r_{1}\left(X_{1} \cup\{e\}\right)+r_{2}\left(X_{2}\right)-l,\right. \\
& \left.\left.r_{1}\left(X_{1} \cup\{e\} \cup p\right)+r_{2}\left(X_{2} \cup p\right)-r_{1}(p)-l\right\}\right\} \\
= & \min \left\{\min \left\{r_{1}\left(X_{1}\right)+r_{2}\left(X_{2}\right), r_{1}\left(X_{1} \cup\{e\}\right)+r_{2}\left(X_{2}\right)-l\right\},\right. \\
& \min \left\{r_{1}\left(X_{1} \cup p\right)+r_{2}\left(X_{2} \cup p\right)-r_{1}(p),\right. \\
& \left.\left.r_{1}\left(X_{1} \cup\{e\} \cup p\right)+r_{2}\left(X_{2} \cup p\right)-r_{1}(p)-l\right\}\right\} \\
= & \min \left\{\min \left\{r_{1}\left(X_{1}\right), r_{1}\left(X_{1} \cup\{e\}\right)-l\right\}+r_{2}\left(X_{2}\right),\right. \\
& \left.\min \left\{r_{1}\left(X_{1} \cup p\right), r_{1}\left(X_{1} \cup\{e\} \cup p\right)-l\right\}+r_{2}\left(X_{2} \cup p\right)-r_{1}(p)\right\} \\
= & \min \left\{r_{P_{1} \downarrow_{e}^{l}}\left(X_{1}\right)+r_{2}\left(X_{2}\right), r_{P_{1} \downarrow_{e}}\left(X_{1} \cup p\right)+r_{2}\left(X_{2} \cup p\right)-r_{1}(b)\right\} \\
= & \left(P_{1} \downarrow \downarrow_{e}^{l} \oplus_{2} P_{2}\right)(X)
\end{aligned}
$$


In [4] the unavoidable minors for connected 2-polymatroids are described as follows:

Let $E$ be a non-empty finite set and choose distinct elements $a, b \notin E$. Let $M_{1}$ be a matroid with groundset $E \cup\{a, b\}$ isomorphic to $U_{1,|E|+1} \oplus U_{0,1}$, where $b$ is the loop, and let $M_{2}$ be a matroid on the same groundset isomorphic to $U_{|E|,|E|+1} \oplus U_{0,1}$, where $a$ is the loop. Then we denote by $\mathcal{S}_{|E|}$ the 2polymatroid $M_{1}+M_{2}$ and define $\mathcal{S}_{0}=U_{1,2}+\left(U_{1,1} \oplus U_{0,1}\right)$.

Theorem 3.43. For every positive integer $n$, there is an integer $r$ such that every connected 2-polymatroid with at least $r$ elements has a minor isomorphic to

1. $U_{1, n}$,

2. $U_{n, n}+U_{n-1, n}$,

3. $U_{n-1, n}$,

4. $U_{n, n}+U_{1, n}$,

5. $2 U_{1, n} ; 2 U_{n-1, n}$ or

6. $\mathcal{Q}_{1} \oplus_{2} \mathcal{Q}_{2} \oplus_{2} \ldots \oplus_{2} \mathcal{Q}_{n}$,

where each $\mathcal{Q}_{i}$ is isomorphic to one of $\mathcal{S}_{0}, \mathcal{S}_{1}, \ldots, \mathcal{S}_{n-1}$ and the groundsets of the $\mathcal{Q}_{i}, \mathcal{Q}_{i+1}$ are disjoint in all but a single rank-1 element.

As stated before this paper defines a minor of a 2-polymatroid $P$ as a polymatroid obtained from $P$ by a series of deletions and contractions. We shall show now that squidging $S_{|E|}$ gives a 2-polymatroid isomorphic to $S_{|E|-1}$, and so we can find a squidge-minor of $S_{|E|}$ that is isomorphic to $S_{1}$.

Theorem 3.44. Let $\mathcal{S}_{|E|}=M_{1}+M_{2}$, where $M_{1}$ and $M_{2}$ are as above, be a 2polymatroid on groundset $E \cup\{a, b\}$. Then for every $e \in E$, the 2-polymatroid $S_{|E|} \downarrow_{e}^{1} \cong S_{|E|-1}$

Proof. We first give the rank function of $S_{|E|}$ by finding the rank functions 
for $M_{1}$ and for $M_{2}$. As $M_{1}=U_{1,|E|+1} \oplus U_{0,1}$ we have

$$
\begin{aligned}
& r_{M_{1}}(A)=1 \text { for all } A \subseteq E \\
& r_{M_{1}}(a)=1 \\
& r_{M_{1}}(b)=0 \\
& r_{M_{1}}(A \cup a)=1 \text { for all } A \subseteq E \\
& r_{M_{1}}(A \cup b)=1 \text { for all } A \subseteq E \\
& r_{M_{1}}(a \cup b)=1 \\
& r_{M_{1}}(A \cup a \cup b)=1 \text { for all } A \subseteq E
\end{aligned}
$$

and the rank function for $M_{2}$ is as follows:

$$
\begin{aligned}
& r_{M_{2}}(A)=|A| \text { for all } A \subseteq E \\
& r_{M_{2}}(a)=0 \\
& r_{M_{2}}(b)=1 \\
& r_{M_{2}}(A \cup a)=|A| \text { for all } A \subseteq E \\
& r_{M_{2}}(A \cup b)= \begin{cases}|A \cup b| & \text { if } A \subsetneq E \\
|E| & \text { if } A=E\end{cases} \\
& r_{M_{2}}(a \cup b)=1 \quad \\
& r_{M_{2}}(A \cup a \cup b)=r_{M_{2}}(A \cup b) \text { for all } A \subseteq E
\end{aligned}
$$

Now we can find the rank function of $M_{1}+M_{2}$

$$
\begin{aligned}
& r_{M_{1}+M_{2}}(A)=|A|+1 \text { for all } A \subseteq E \\
& r_{M_{1}+M_{2}}(a)=1 \\
& r_{M_{1}+M_{2}}(b)=1 \\
& r_{M_{1}+M_{2}}(A \cup a)=|A|+1 \text { for all } A \subseteq E \\
& r_{M_{1}+M_{2}}(A \cup b)=\left\{\begin{array}{ll}
|A \cup b|+1 & \text { if } A \subsetneq E \\
|E|+1 & \text { if } A=E
\end{array} \quad \text { for all } A \subseteq E\right. \\
& r_{M_{1}+M_{2}}(a \cup b)=2 \\
& r_{M_{1}+M_{2}}(A \cup a \cup b)=r_{M_{1}+M_{2}}(A \cup b) \text { for all } A \subseteq E
\end{aligned}
$$

Now that we have the rank function of $M_{1}+M_{2}$ we find the rank function 
of $\left(M_{1}+M_{2}\right) \downarrow_{e}^{1}$ for $e \in E$.

$r_{\left(M_{1}+M_{2}\right) \downarrow_{e}^{1}}(A)=\min \left\{r_{M_{1}+M_{2}}(A), r_{M_{1}+M_{2}}(A \cup\{e\})-1\right\}$ for all $A \subseteq E$

$r_{\left(M_{1}+M_{2}\right) \downarrow_{e}^{l}}(a)=\min \left\{r_{M_{1}+M_{2}}(a), r_{M_{1}+M_{2}}(a \cup\{e\})-1\right\}$

$r_{\left(M_{1}+M_{2}\right) \downarrow_{e}}(b)=\min \left\{r_{M_{1}+M_{2}}(b), r_{M_{1}+M_{2}}(b \cup\{e\})-1\right\}$

$r_{\left(M_{1}+M_{2}\right) \downarrow_{e}^{l}}(A \cup a)=\min \left\{r_{M_{1}+M_{2}}(A \cup a), r_{M_{1}+M_{2}}(A \cup a \cup\{e\})-1\right\}$ for all $A \subseteq E$

$r_{\left(M_{1}+M_{2}\right) \downarrow_{e}^{l}}(A \cup b)=\min \left\{r_{M_{1}+M_{2}}(A \cup b), r_{M_{1}+M_{2}}(A \cup b \cup\{e\})-1\right\}$

$r_{\left(M_{1}+M_{2}\right) \downarrow_{e}^{l}}(a \cup b)=\min \left\{r_{M_{1}+M_{2}}(a \cup b), r_{M_{1}+M_{2}}(a \cup b \cup\{e\})-1\right\}$

$r_{\left(M_{1}+M_{2}\right) \downarrow_{e}^{l}}(A \cup a \cup b)=\min \left\{r_{M_{1}+M_{2}}(A \cup b), r_{M_{1}+M_{2}}(A \cup\{e\} \cup b)-1\right\}$

Which gives

$$
\begin{aligned}
& r_{\left(M_{1}+M_{2}\right) \downarrow_{e}^{l}}(A)=|A|+1 \text { for all } A \subseteq E-\{e\} \\
& r_{\left(M_{1}+M_{2}\right) \downarrow_{e}^{l}}(\{a\})=1 \\
& r_{\left(M_{1}+M_{2}\right) \downarrow_{e}^{l}}(\{b\})=1 \\
& r_{\left(M_{1}+M_{2}\right) \downarrow_{e}^{l}}(A \cup\{b\})= \begin{cases}|A \cup\{b\}|+1 & \text { if } A \subsetneq E \\
|E|+1 & \text { if } A=E\end{cases} \\
& r_{\left(M_{1}+M_{2}\right) \downarrow_{e}^{l}}(A \cup\{a\})=|A|+1 \text { for all } A \subseteq E-\{e\} \\
& r_{\left(M_{1}+M_{2}\right) \downarrow_{e}^{l}}(\{a, b\})=2 \\
& r_{\left(M_{1}+M_{2}\right) \downarrow_{e}^{l}}(A \cup\{a, b\})=r_{\left(M_{1}+M_{2}\right) \downarrow_{e}^{l}}(A \cup b)
\end{aligned}
$$

Notice that when $|A \cup b|=|E-\{e\}|$, we have that $r_{\left(M_{1}+M_{2}\right) \downarrow_{e}^{l}}(A \cup b)=$ $|E-\{e\}|+1$ so we can write

$$
r_{\left(M_{1}+M_{2}\right)_{e}^{l}}(A \cup a)= \begin{cases}|A \cup\{b\}|+1 & \text { if }|A \cup\{b\}| \leq|E-\{e\}| \\ |E-\{e\}|+1 & \text { if }|A|>|E-\{e\}|\end{cases}
$$

Which is the rank function for $S_{|E-\{e\}|}$. 


\section{Chapter 4}

\section{Graphic Connectivity Functions}

In [11] Seymour proved that that there is an algorithm for establishing when a matroid is graphic in $p(n)$ rank evaluations where $p$ is a particular polynomial and $n$ is the size of the groundset of the matroid. He also proved that there is no such algorithm for establishing when a matroid is binary. In this section we prove a similar theorem for graphic connectivity functions, that is we prove the following theorem:

Theorem 4.1. There is some polynomial $p$ such that, given an arbitrary connectivity function $\lambda$, we are able to establish whether or not $\lambda$ is graphic in at most $p(n)$ evaluations of the connectivity function, where $n$ is the size of the set on which $\lambda$ is based.

Throughout this section we are working with the vertex connectivity of the graph, and so, when we talk about the connectivity function of $G$, we mean the connectivity function, $\gamma_{G}$, of graph $G$ defined as follows:

$$
\gamma_{G}(X)=|V(X)|+|V(E(G)-X)|-|V(E(G))|
$$

for all $X \subseteq E(G)$. Recall that $\gamma(X)$ counts the number of vertices $X$ has in common with $E(G)-X$.

Definition 4.2. A connectivity function, $\lambda$, is graphic if there is some graph $G$ such that $\lambda=\gamma_{G}$.

We shall often abbreviate $\gamma_{G}$ to $\gamma$ when it is clear what we mean from the context. 
As previously stated, this section aims to provide a polynomial-time algorithm for deciding whether or not a connectivity function is graphic. To do this we first suppose that we have a graphic connectivity function, $\gamma$. The first part of this section gives a method for finding the edge adjacencies of the graph (or graphs) that has $\gamma$ as its connectivity function. The second part gives a method for identically building the graph from its edge adjacencies. The third section first gives an algorithm that builds a graph which would have $\lambda$ as its connectivity function were $\lambda$ graphic. At some stages in this we may be able to identify $\lambda$ as not graphic. However, it may be that $\lambda$ is not graphic but we do not find this from the algorithm. The section then gives a method for deciding whether or not $\lambda$ is the connectivity function of the graph, $G$, we just built. If $\lambda$ were graphic, then $G$ would have connectivity function $\lambda$, so from this we are able to identify exactly when $\lambda$ is graphic.

We call $e \in E(G)$ a leaf if $e$ is an edge that is incident with a vertex of degree one. It can easily be seen that a connectivity function cannot tell the difference between loops and leaves. The following example demonstrates this:



In $G_{1}$ the edge $c$ is a leaf and $d$ is a loop. In $G_{2}$ this is reversed. Both $G_{1}$ and $G_{2}$ have the following connectivity function: $\gamma(\{a\})=2, \gamma(\{b\})=$ $1, \gamma(\{c\})=1, \gamma(\{d\})=1, \gamma(\{a, b\})=2, \gamma(\{a, c\})=2, \gamma(\{a, d\})=$ $1, \gamma(\{b, c\})=1, \gamma(\{b . d\})=2, \gamma(\{c, d\})=2$. The remaining values can be found by symmetry.

The connectivity function is also based on the edges of the graph and cannot tell whether or not a graph contains isolated vertices, that is single vertices with no edges of $E(G)$ incident with them. In other words we have the following lemma:

Lemma 4.3. Let $\gamma$ be a graphic connectivity function, then $\gamma$ is the connectivity function of some loopless graph with no isolated vertices. 
Therefore, throughout this chapter we assume that all graphs we talk about are loopless graphs with no isolated vertices, unless stated otherwise.

Because we are interested in the vertex-connectivity in this section, and that is based on the set of edges of the graph, the sets in this section will generally be sets of edges. To simplify notation, when we are given a graph, $G$, we shall use $|G|$ to mean $|E(G)|$.

\subsection{Facts About Connectivity Functions of Graphs}

Given a graphic connectivity function we are able to find the edge adjacencies of the graphs with that connectivity function. This section gives a method for doing this.

Definition 4.4. Let $G$ be a graph and $e, f \in E(G)$, then:

1. $P$ is a parallel set of $G$ if $P \subseteq E(G)$ and every pair of edges in $P$ are parallel.

2. $P$ is a parallel class if $P$ is a maximal parallel set in $G$.

3. $P$ is a parallel-leaf if $P$ is a parallel class and for every $e \in P$ we have $e$ is a leaf edge in $G \backslash(P-e)$.

4. If $A \subseteq E(G)$, then $V(A)$ is the collection of vertices incident with an element of $A$.

5. If $A \subseteq E(G)$, then the subgraph induced by $A$ is the graph $(V(A), A)$.

6. If $A \subseteq E(G)$, then we say $A$ is connected if the subgraph induced by $A$ is connected.

7. A connected component of a graph $G$ is a maximal connected subgraph of $G$.

8. A separator of $G$ is the (entire) set of edges of a collection of connected components of $G$.

9. An isolated egde of $G$ is an edge of $G$ that is a separator of $G$. 
10. An $m$-star of $G$ is a set $S \subseteq E$ such that all edges of $S$ are incident with a common vertex and $|S|=m$. We call a subset of the edges of $G$ a star if it is an $m$-star for some $m$.

11. A complete star of $G$ is the collection of all edges incident with a specified vertex.

12. A partial star of $G$ is a star of $G$ that is not a complete star of $G$.

13. If $S$ is a star and every edge of $S$ is incident with vertex $v$ then $v$ is a centre of $S$.

There is a unique complete star with centre $v$ and, if $S$ is a star that is not a parallel set, then the centre of $S$ is unique.

Definition 4.5. Let $\left\{a_{1}, \ldots, a_{m}\right\}$ be a collection of edges in $G$. We say that a vertex $v_{i}$ is full in $G$ with respect to $\left\{a_{1}, \ldots, a_{m}\right\}$ if the complete star with centre $v_{i}$ is a subset of $\left\{a_{1}, \ldots, a_{m}\right\}$.

Throughout this section, when we refer to a graph, G, we shall assume that $G$ has edgeset $E$ and connectivity function $\gamma$, unless stated otherwise. As stated before we shall also assume that $G$ is loopless and contains no isolated vertices.

It can easily be seen that, for a graph $G$, if $A \subseteq E$ then $A$ is a separator of $G$ if, and only if, $\gamma(A)=0$.

The following lemma is obvious from noting that the connectivity of a set is the number of vertices that set has in common with its complement.

Lemma 4.6. An edge e of a graph $G$ has connectivity 1 or 0 if, and only if, $e$ is a leaf. Otherwise $\gamma(e)=2$. Moreover, if $\gamma(e)=0$ then $e$ induces a connected component of $G$.

Lemma 4.7. Let $G$ be a graph with at least three vertices and connectivity function $\gamma$; and suppose we have two non-isolated edges e, $f$ of $G$ neither of which induces a connected component of $G$. Then:

(i) if $e$ and $f$ are not leaves, then $0 \leq \gamma(\{e, f\}) \leq 3$ if, and only if, $e$ and $f$ are adjacent. 
(ii) if exactly one of e or $f$ is a leaf, then $0 \leq \gamma(\{e, f\}) \leq 2$ if, and only if, $e$ and $f$ are adjacent.

(iii) if both $e$ and $f$ are leaves, then $0 \leq \gamma(\{e, f\}) \leq 1$ if, and only if, $e$ and $f$ are adjacent.

Proof.

(i) Suppose that $e$ and $f$ are adjacent. Then $|V(\{e, f\})| \leq 3$ and so $\gamma(\{e, f\}) \leq 3$

Suppose that $e$ and $f$ are not adjacent. Then $|V(\{e, f\})|=4$, as neither $e$ nor $f$ is a loop. As neither $e$ nor $f$ is a leaf and neither edge induces a connected component of $G$, no vertex in $V(\{e, f\})$ is full with respect to $\{e, f\}$ and therefore $V(E-\{e, f\})=V(E)$. This means that $\gamma(\{e, f\})=|V(\{e, f\})|=4$.

(ii) Suppose that $e$ and $f$ are adjacent. Then $|V(\{e, f\})| \leq 3$ and at least one of those vertices is full with respect to one of $e$ and $f$. Therefore $\gamma(\{e, f\}) \leq 2$.

Suppose that $e$ and $f$ are not adjacent. Then $|V(\{e, f\})|=4$, as neither $e$ nor $f$ is a loop. As exactly one of $e$ and $f$ is a leaf and neither edge induces a connected component of $G$, exactly one vertex in $V(\{e, f\})$ is full with respect to $\{e, f\}$ and therefore $V(E-\{e, f\})=V(E)-1$. This means that $\gamma(\{e, f\})=|V(\{e, f\})|-1=3$.

(iii) Suppose that $e$ and $f$ are adjacent. Then $|V(\{e, f\})| \leq 3$, and one of those vertices is full with respect to $e$, and one is full with respect to $f$. Therefore $\gamma(\{e, f\}) \leq 1$.

Suppose that $e$ and $f$ are not adjacent. Then $|V(\{e, f\})|=4$, as neither $e$ nor $f$ is a loop. As both of $e$ and $f$ are leaves and neither edge induces a connected component of $G$, exactly two vertices in $V(\{e, f\})$ are full with respect to $\{e, f\}$ and therefore $V(E-\{e, f\})=V(E)-2$. This means that $\gamma(\{e, f\})=|V(\{e, f\})|-2=2$. 
Lemmas 4.6 and 4.7 together enable us to identify, for every pair of edges $a$ and $b$, whether or not $a$ and $b$ are adjacent by evaluating $\gamma(\{a\}), \gamma(\{b\})$ and $\gamma(\{a, b\})$

Definition 4.8. A graph is called simple if it contains no loops or parallel edges. An underlying simple graph $G_{s}$ of a graph $G$ is the graph obtained by deleting all loops of $G$ and all but one member of each parallel class of $G$.

To be able to construct an underlying simple graph of $G$ we need to know which edges of $G$ are loops, and which edges are in a parallel class. As we are assuming that $G$ contains no loops we need only identify parallel classes. The following section will, where possible, give a method for doing this.

\subsubsection{Parallel Edges}

Definition 4.9. Let $\gamma$ be a graphic connectivity function on $E$ and $e, f$ be distinct edges of $E$. We call the pair $\{e, f\}$ parallel ambiguous if there exists graphs $G_{1}$ and $G_{2}$ on set $E$ such that $G_{1}$ and $G_{2}$ both have connectivity function $\gamma$ and the pair $\{e, f\}$ is parallel in $G_{1}$ but not $G_{2}$.

In this section we shall prove the following Lemma:

Lemma 4.10. Let $\gamma$ be a graphic connectivity function on set $E$ and let $a, b \in E$. If $\{a, b\}$ is parallel ambiguous then the only graphs with connectivity function $\gamma$ must contain a connected component containing $a$ and $b$ that is isomorphic to one of:
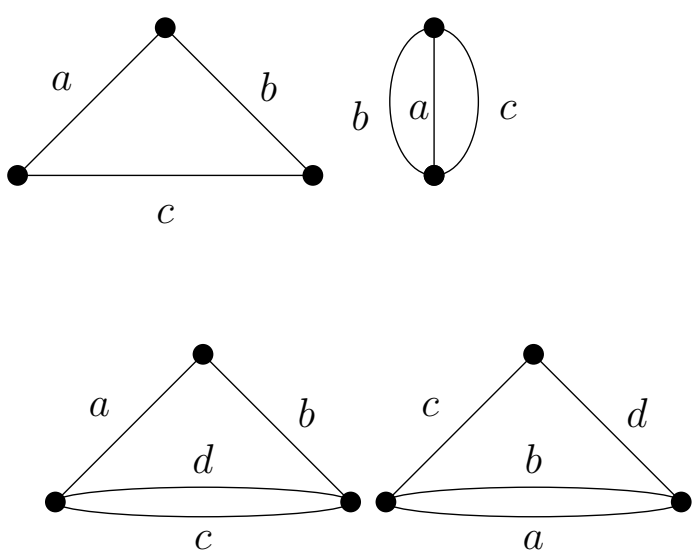
We shall also give a way to identify parallel edges when they are not parallel ambiguous.

Definition 4.11. A triangle of a graph $G$ is a subgraph of $G$ isomorphic to $K_{3}$. A parallel triangle is a loopless subgraph of $G$ whose underlying simple graph is a triangle. We say that an edge, $e$, in a parallel triangle is a single side of the parallel triangle if it is not parallel to any other element of $G$.

Lemma 4.12. Let $G$ be a graph with at least three edges, and let $e, f$ be distinct edges of $G$, such that $\gamma(\{e\})=\gamma(\{f\})=2$ and $\gamma(\{e, f\}) \leq 2$. Let $G^{\prime}$ be the connected component of $G$ containing e and $f$. Then $\gamma(\{e, g\})=$ $\gamma(\{f, g\})$ for all $g \in E(G)-\{e, f\}$ if, and only if, $\{e, f\}$ is a parallel pair or $G^{\prime}$ is a parallel-triangle with e and $f$ as single sides.

Proof. Clearly if $e, f$ are parallel or $G^{\prime}$ is a parallel triangle with $e$ and $f$ single sides then for all $g \in E(G)-\{e, f\}$ we have $\gamma(\{e, g\})=\gamma(\{f, g\})$.

Suppose that $e$ and $f$ are not parallel to each other. As $\gamma(\{e\})=\gamma(\{f\})=2$ neither $e$ nor $f$ can be a leaf. As $\gamma(\{e, f\}) \leq 2$ and $e$ and $f$ are not leaves, $e$ and $f$ must be adjacent. Let $V(\{e\})=\left\{v_{1}, v_{2}\right\}$ and $V(\{f\})=\left\{v_{2}, v_{3}\right\}$. As $e$ and $f$ are not parallel $v_{1} \neq v_{3}$, and as $\gamma(\{e, f\}) \leq 2$, and $e$ and $f$ are not leaves, the vertex $v_{2}$ must be full with respect to the pair $\{e, f\}$. This vertex is shown in red on the diagram below.

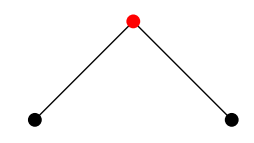

Suppose $\gamma(\{e, g\})=\gamma(\{f, g\})$ for all $g \in E(G)$. Then every edge that is adjacent to $e$ must also be adjacent to $f$ and vice verse, for suppose that $g$ were adjacent to $e$ but not to $f$. If $g$ is a leaf then, by Lemma 4.7, $\gamma(\{e, g\}) \leq$ 2 and $\gamma(\{f, g\})>2$. If $g$ is not a leaf then, by Lemma 4.7, $\gamma(\{e, g\}) \leq 3$ and $\gamma(\{f, g\})>3$. Both alternatives lead to a contradiction, and so every edge that is adjacent to $e$ must also be adjacent to $f$. Similarly every edge that is adjacent to $f$ must also be adjacent to $e$. The only way an edge $g \in E(G)-\{e, f\}$ can be adjacent to $e$ is if it is incident with $v_{1}$ as $v_{2}$ is full with respect to $\{e, f\}$, and, for the same reason, the only way for an edge to be adjacent to $f$ is for it to be incident with $v_{3}$. Therefore every edge that is adjacent to $e$ must be incident with $v_{1}$ and $v_{3}$, and every edge that 
is adjacent to $f$ is also adjacent to $e$. This means that $G^{\prime}$ must be a parallel triangle.

The following two examples illustrate that $\gamma$ does not always uniquely define $G$; in both examples the pair $\{a, b\}$ is parallel ambiguous.



It is easy to see that both $G_{1}$ and $G_{2}$ have the following connectivity function: $\gamma(\{a\})=2, \gamma(\{b\})=2, \gamma(\{c\})=2, \gamma(\{a, b\})=2, \gamma(\{a, c\})=2, \gamma(\{b, c\})=$ $2, \gamma(\{a, b, c\})=0$,

The following two graphs also have the same connectivity functions:

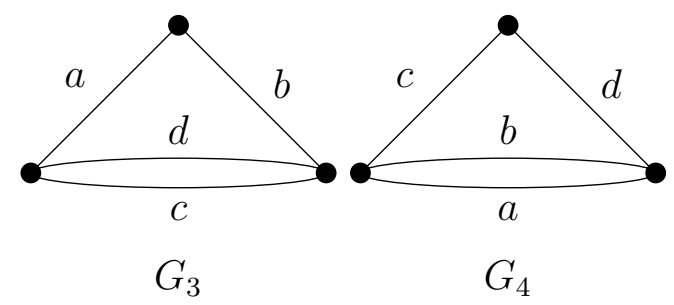

Again, we can see that both $G_{3}$ and $G_{4}$ have the following connectivity function: $\gamma(\{a\})=2, \gamma(\{b\})=2, \gamma(\{c\})=2, \gamma(\{d\})=2, \gamma(\{a, b\})=$ $2, \gamma(\{a, c\})=3, \gamma(\{a, d\})=3, \gamma(\{b, c\})=3, \gamma(\{b . d\})=3, \gamma(\{c, d\})=2$. The remaining values can be found by symmetry.

We shall now show that the only way we cannot tell from a connectivity function when two edges are parallel are if those two edges are contained in a connected component of isomorphic to one of $G_{1}, \ldots, G_{4}$ above.

Lemma 4.12 gives a way to identify when two distinct adjacent edges $a$ and $b$ are either parallel, or are single sides in a connected component of $G^{\prime}$ that is a parallel triangle. Our next lemma gives a way to distinguish between these two cases. 
Lemma 4.13. Suppose that $G^{\prime}$ is a connected component of $G$ containing two distinct non-leaf edges $a$ and $b$ and suppose that either $G^{\prime}$ is a parallel triangle with $a$ and $b$ as single sides, or $a$ is parallel to $b$ in $G$ (in this case $G^{\prime}$ need not be a parallel triangle) and suppose that $\left|G^{\prime}\right|>4$. Then a and $b$ are parallel in $G$ if, and only if, one of the following holds:

1. $\gamma(\{a, b\})<2$

2. there exists an e such that $\gamma(\{e\})=1$ and $e$ is adjacent to $a$ or $b$

3. there is some non-isolated e such that $\gamma(\{a, e\})=\gamma(\{b, e\})=2$

4. there are some e, $f$ adjacent to $a$ and $b$ respectively such that $\gamma(\{e, f\}) \neq$ 2

Proof. Suppose that $\{a, b\}$ are not parallel. Then $a$ and $b$ are single sides of a parallel triangle as shown below in $G_{1}$

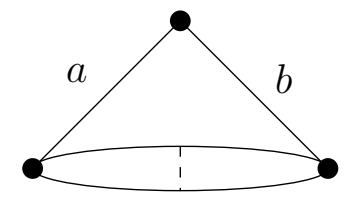

$G_{1}$

It is clear that if we have this situation, all of 1.-4. fail.

Suppose that none of 1.-4. hold. For the purposes of contradiction suppose that $a$ and $b$ are parallel. We know that $\{a, b\}$ cannot be a parallel leaf as $\gamma(\{a, b\}) \geq 2$. Also, as there is no $e \in E$ such that $\gamma(\{a, e\})=\gamma(\{b, e\})=2$, there can be no $e \in E$ such that $e$ is in a parallel class with $a$ and $b$. The diagram, $G_{2}$, below gives the form of the situation we must, therefore, be in, where the dashed lines show edges that must be adjacent to $a$ and $b$. However, the diagram does not show how these dashed edges interact with each other, or any edges of the graph that are not $a$ or $b$.

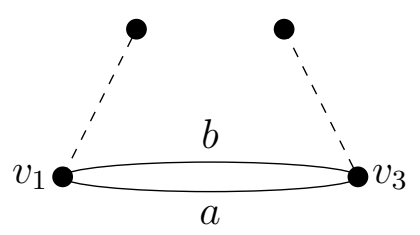


Let one of the edges incident with $v_{1}$ be called $e$ and one of the edges incident with $v_{3}$ be called $f$. Note that as there can be no edges parallel to $a$ and $b$, the edges $e$ and $f$ must be distinct and not parallel. Neither $e$ nor $f$ can be a leaf in $G$, as otherwise 2. would hold. As 4. does not hold, it must be that $\gamma\{e, f\}=2$, and therefore, by Lemma 4.7, $e$ and $f$ must be adjacent. Moreover if $v_{2} \in V(e) \cap V(f)$, then $v_{2}$ is full with respect to $e$ and $f$. Therefore we are now in the situation shown below in $G_{3}$, where the red dot indicates a full vertex.

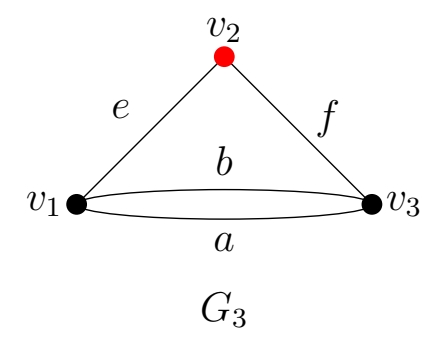

As $\left|G^{\prime}\right| \geq 5$, there is some edge $g$ incident with some vertex in $\left\{v_{1}, v_{2} \cdot v_{3}\right\}$. It cannot be incident with $v_{2}$ as this vertex is full with respect to $\{e, f\}$, therefore, without loss of generality, assume that $g$ is incident with $v_{1}$. The edge $g$ cannot be a leaf, as otherwise 2 . would hold, and $g$ cannot be parallel to $a, b$ as otherwise 3. would hold. Therefore we now are in the situation illustrated by $G_{4}$ below, where the green vertex is not full with respect to $g$ :

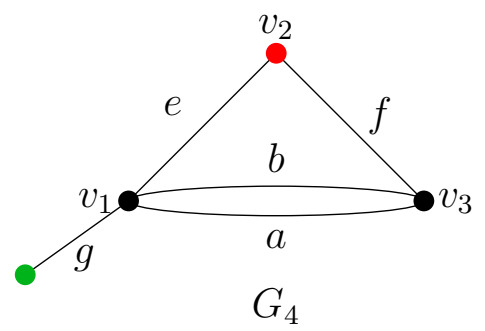

Therefore $\gamma\{g, f\}=4$, and so 4. holds, a contradiction. Therefore assuming that none of 1.-4. held and that $\{a, b\}$ were parallel led to a contradiction, so if none of 1.-4. hold, $G^{\prime}$ must be a parallel triangle with $a$ and $b$ as single sides.

Suppose that $G^{\prime}$ is a connected component of $G$ containing two adjacent edges, $a$ and $b$, and exactly one other edge. We are able to find out when this case occurs by finding the elements adjacent to one or both of $a$ and $b$. 
If there is more than one such element, or no such element then $\left|G^{\prime}\right| \neq 3$, a contradiction, and if there is only one such element, say e, $\left|G^{\prime}\right|=3$ if, and only if, $\gamma(\{a, b, e\})=0$.

Suppose that $G^{\prime}$ is a connected component of $G$ containing two edges $a$ and $b$, which are adjacent, and exactly two other edges. We are able to find out when this case occurs by finding the elements adjacent to one or both of $a$ and $b$. If there are more than two such elements then $\left|G^{\prime}\right|>4$, and if there are exactly two such elements, say $e, f$, then $\left|G^{\prime}\right|=4$ if, and only if, $\gamma(\{a, b, e, f\})=0$. If there is only one such element, $e$, and it is not a leaf then we can find the elements adjacent to $e$, and if there is more than one element adjacent to $e$ that is not $a$ or $b$, then $\left|G^{\prime}\right| \geq 4$ and if there is exactly one such element, say $f$, then $\left|G^{\prime}\right|=4$ if, and only if, $\gamma(\{a, b, e, f\})=0$.

Therefore, when $\left|G^{\prime}\right| \geq 3$ we are now in a position to identify when two edges, $a$ and $b$, are either single edges in a parallel triangle that makes up an entire connected component of $G$ or parallel. Given we know that $a$ and $b$ are in one of these two categories, we are able to tell if the connected component of $G$ containing $a$ and $b$ has more than four edges. If it does we are able to tell when $a$ and $b$ are parallel. Suppose that the connected component containing $a$ and $b$ contains exactly three edges. Then it must be one of the graphs shown below (with a possible relabelling of $c$ ):

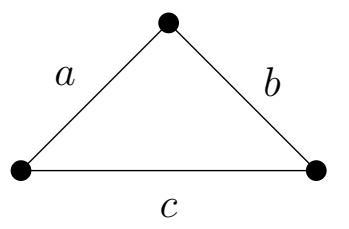

$G_{1}$



$G_{2}$

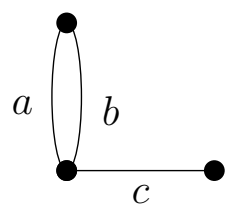

$G_{3}$

We can tell when we are in situation $G_{3}$, by noting that this contains an edge, $c$, such that $\gamma(\{c\})=1$.

Suppose that the connected component containing $a$ and $b$ has four edges. Then the only options for this connected component are the ones shown below: 


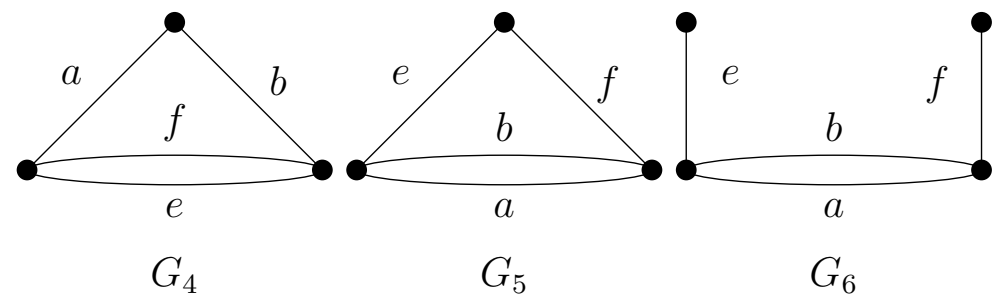

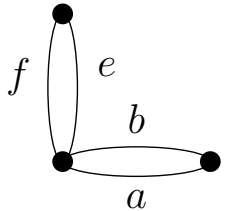

$G_{7}$

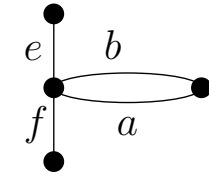

$G_{8}$

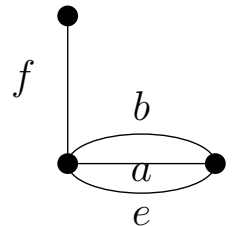

$G_{9}$


All these graphs, except $G_{4}$ and $G_{5}$ can be told apart by their connectivity functions, and, more importantly, the connectivity function of $G_{4}$ and $G_{5}$ is not equal to the connectivity function of any of the other graphs.

Lemma 4.10. Let $\gamma$ be a graphic connectivity function on set $E$ and let $a, b \in$ $E$. If $\{a, b\}$ is parallel ambiguous then the only graphs with connectivity function $\gamma$ must contain a connected component containing $a$ and $b$ that is isomorphic to one of:
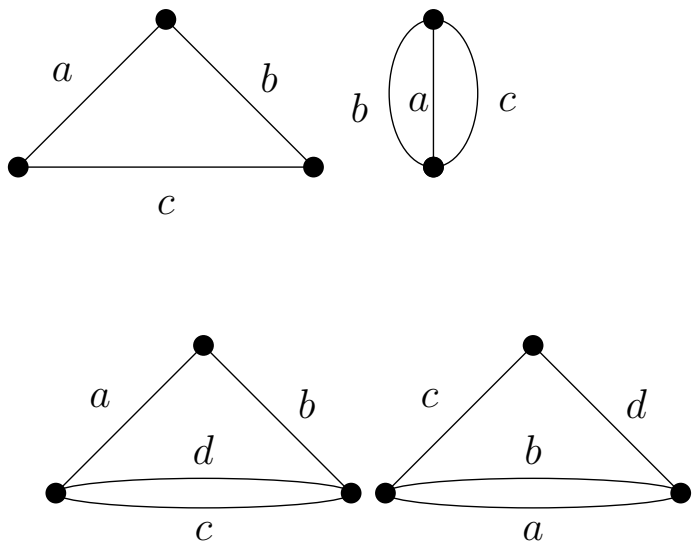
Proof. Clearly if $\left|G^{\prime}\right| \leq 2$, where $G^{\prime}$ is the connected component of $G$ containing $a$ and $b$, then we can tell when $a$ and $b$ are parallel. So suppose $\left|G^{\prime}\right| \geq 3$. Given two edges $a$ and $b$, Lemmas 4.6 and 4.7 enable us to tell, from the connectivity function, whether $a$ and $b$ are adjacent. As the graph is loop free, if $a$ and $b$ are parallel then $\gamma(\{a\})=2, \gamma(\{b\})=2$ and $\gamma(\{a, b\}) \leq 2$. This means that we can apply Lemma 4.12 which gives us a condition on the connectivity function that is sufficient to determine whether $\{a, b\}$ lies within the class of edges that are either parallel or single sides of a parallel triangle that is a connected component of $G$. If $a$ and $b$ are parallel ambiguous then the ambiguity lies between $a$ and $b$ being parallel and $a$ and $b$ being single sides of a connected component that is a parallel triangle. Lemma 4.13 then shows that, given a connectivity function, there can be no ambiguity unless $\left|G^{\prime}\right|=3$ or 4 . The remarks following Lemma 4.13 show that the cases where $\left|G^{\prime}\right|=3$ and when $\left|G^{\prime}\right|=4$ can be resolved by considering the connectivity function in all but the two cases given above.

Furthermore it is clear that whether a pair is parallel ambiguous, and if not, whether it is parallel, can be determined in polynomial time.

\subsection{Building a Graph from the Edge Adja- cencies}

Suppose that we know the collection of stars of a simple graph. This is the same as knowing the vertices of the graph and the edges incident to those vertices and so we can build the graph. In [17] a polynomial time algorithm for constructing a graph given the edge adjacencies is given. However, this algorithm only guarantees that the graph is built up to isomorphism (although, in most cases, it will be identically built). We modify this algorithm slightly so that we construct the graph identically. Other papers on building the graph from the edge adjacencies exist (equivalently building the graph from what is known as its line graph), such as [5] and [15], but, from a brief scan of the literature, these papers did not seem to guarantee building the graph up to identity.

Let $G$ be a simple graph with edge set $E$ and let $\mathcal{A}$ be the set of all pairs of 
elements $\{e, f\} \subseteq E$ that are edge adjacent in $G$.

Let $\mathcal{P}$ be the collection of subsets of $E$ such that for every $P \in \mathcal{P}$ every pair of elements of $P$ is in $\mathcal{A}$ and $P$ is maximal with respect to this property.

If $G$ contains a connected component isomorphic to $K_{3}$ or $Y$ (as given below), then we cannot tell from the edge adjacencies whether the connected component is isomorphic to $K_{3}$ or to $Y$.

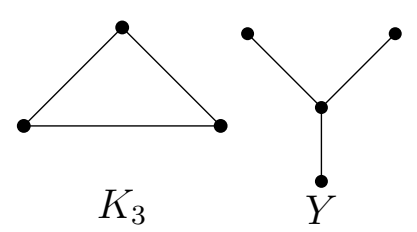

If $G$ contains a connected component isomorphic to one of the graphs given below, then from the edge adjacencies we can tell which of the following graphs the connected component is isomorphic to but we cannot tell in which way the edges are distributed over the connected component.

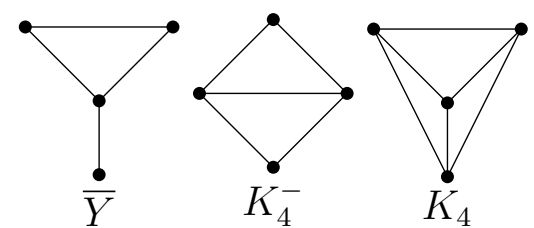

Lemma 4.14. If $P \in \mathcal{P}$ then either $P$ is a complete star or $P$ is a triangle. What is more $\mathcal{P}$ contains all complete stars on more than two elements and all triangles.

Proof. Let $Q$ be a partial star. Then there is an edge $e \in E-Q$ such that $e$ is adjacent to all edges in $Q$. Therefore $Q \notin \mathcal{P}$, by the maximality of sets in $\mathcal{P}$.

Let $Q$ be a connected set that is not a star or triangle in $G$. Then $Q$ must contain a path of length 3 . The first and least elements in this path cannot be adjacent, as otherwise $Q$ would be a star or a triangle, and therefore $Q \notin \mathcal{P}$. Suppose there is a complete star, $S$, such that $|S|>2$ and $S \notin \mathcal{P}$. By the definition of $\mathcal{P}$ there must be some $P \in \mathcal{P}$ such that $S \subsetneq P$. Therefore there is an $f \in E-S$ such that $S \cup\{f\} \subseteq P$. The edge $f$ cannot be incident with more than two vertices in $|V(S)|$, and $f$ cannot be incident with the 
centre of $S$, therefore $f$ can be adjacent to only two edges of $S$. Therefore $S \cup\{f\} \nsubseteq P$; a contradiction.

If there were some triangle, $T$, of $G$, not contained in $\mathcal{P}$ then either not every pair of elements of $T$ is in $\mathcal{A}$ contradicting the fact that $T$ is a triangle, or there is some other edge adjacent to every pair of elements of $T$, a contradiction to $G$ being simple.

The set $\mathcal{P}$ may not contain all complete stars with two elements, as these may be subsets of a triangle. We need a way of listing all stars of $E$, so we need to be able to distinguish between 3-element stars and triangles. Our next lemmas will give a method for doing this except in the five ambiguous cases described above. Let $\mathcal{P}_{3}$ be the collection of 3-element sets of $\mathcal{P}$ and let $\mathcal{P}-\mathcal{P}_{3}=\mathcal{P}^{*}$.

Lemma 4.15. Suppose that $P \in \mathcal{P}_{3}$ and there is some $P^{\prime} \in \mathcal{P}^{*}$ that has a non-zero intersection with $P$. Then either $\left|P \cap P^{\prime}\right|=1$ or $\left|P \cap P^{\prime}\right|=2$. If $\left|P \cap P^{\prime}\right|=1$ then $P$ is a star and if $\left|P \cap P^{\prime}\right|=2$ then $P$ is a triangle.

Proof. If $\left|P \cap P^{\prime}\right|>2$, then $P \subsetneq P^{\prime}$, contradicting the maximality of $P$. Suppose that $P$ is not a star; then $P$ must be a triangle. Therefore every vertex in $V(P)$ would be incident with exactly two edges of $P$. As $P^{\prime}$ is a star that shares an edge with $P$, the set $V\left(P^{\prime}\right)$, must also share two vertices with $V(P)$ and one of these vertices must be the centre of the star $P^{\prime}$. This means that $P$ must share two edges with $P^{\prime}$ as $P^{\prime}$ is a complete star. Therefore $\left|P \cap P^{\prime}\right|=2$.

If $\left|P \cap P^{\prime}\right|=2$ then there is some vertex of $P$ at which exactly two edges of $P$ meet, for consider the vertex where the two edges of $P \cap P^{\prime}$ meet (by the definition of $P$ they must meet at some vertex). If this vertex had more than two edges of $P$ incident with it, then $P^{\prime}$ would not be maximal. Therefore there is some vertex of $P$ at which exactly two edges of $P$ meet and so $P$ must be a triangle.

For the remainder of this section we shall suppose that $P \in \mathcal{P}_{3}$ and there is no member of $\mathcal{P}^{*}$ whose intersection with $P$ is non-empty. We shall also assume that $\mathcal{A}$ is not the collection of edge adjacencies of any of $K_{4}, K_{4}^{-}, \bar{Y}, K_{3}$ or $Y$. 
Lemma 4.16. Suppose that there is a member, $P^{\prime}$, of $\mathcal{P}_{3}$ such that $\left|P \cap P^{\prime}\right|=$ 1. Then either $P$ and $P^{\prime}$ are both 3-element stars or both triangles.

Proof. Suppose that $P$ is a star and $P^{\prime}$ is a triangle and suppose that $\mid P \cap$ $P^{\prime} \mid=1$. Then $P$ and $P^{\prime}$ must share exactly two vertices and one of those vertices must be the centre vertex, $v$, of the star $P$. But as $P^{\prime}$ is a triangle there must be two edges incident with $v$ in $P^{\prime}$ and as $P$ is a complete star at vertex $v$ both those edges must be in $P$, so $\left|P \cap P^{\prime}\right|=2$, a contradiction. Similarly $P$ cannot be a triangle while $P^{\prime}$ is a star.

Lemma 4.17. Let $P^{\prime} \in \mathcal{P}_{3}$ be such that $\left|P \cap P^{\prime}\right|=1$. Then if there is some edge, e of $P$ such that for all $f \in P^{\prime}-P$ we have $\{e, f\} \notin \mathcal{A}$ then $P$ is a star.

Proof. Suppose that $P$ were a triangle. As $\left|P \cap P^{\prime}\right|=1$ we must also have $P^{\prime}$ is a triangle, and the two triangles must meet along a single edge. Then every edge of $P^{\prime}-P$ is adjacent to an edge of $P$, a contradiction.

Lemma 4.18. Let $P^{\prime} \in \mathcal{P}_{3}$ be such that $\left|P \cap P^{\prime}\right|=1$ and suppose that for every edge $e$ of $P$ there is some $f \in P^{\prime}-P$ such that $\{e, f\} \in \mathcal{A}$. Suppose that there is an element, $P^{\prime \prime} \nsubseteq P \cup P^{\prime}$, of $\mathcal{P}$ such that $\left|P \cap P^{\prime \prime}\right|=2$ or $\left|P^{\prime} \cap P^{\prime \prime}\right|=2$. Then $P$ is a triangle or $G^{\prime} \cong K_{4}$, where $G^{\prime}$ is the connected component of the graph which contains $P$. If no such $P^{\prime \prime}$ exists then $P$ is a star or $G^{\prime} \cong K_{4}^{-}$.

Proof. We know that, as $P, P^{\prime} \in \mathcal{P}_{3}$ and $\left|P \cap P^{\prime}\right|=1$, either $P$ and $P^{\prime}$ are both triangles, or both stars. If, for every edge $e$ of $P$, there is some $f \in P^{\prime}-P$ such that $\{e, f\} \in \mathcal{A}$, then, when we restrict the edges in our graph to $P \cup P^{\prime}$ and our vertices to $V\left(P \cup P^{\prime}\right)$, we get a graph isomorphic to $K_{4}^{-}$. The possible ways this may arise are shown below with the edges of $P$ shown in red and those of $P^{\prime}$ shown in blue, the common edge of $P$ and $P^{\prime}$ is shown in purple.

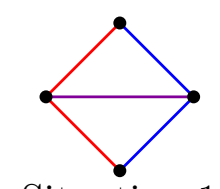

Situation 1

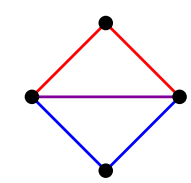

Situation 2 
Proof that, if there exists a $P^{\prime \prime} \in \mathcal{P}$ such that $\left|P \cap P^{\prime \prime}\right|=2$ or $\left|P^{\prime} \cap P^{\prime \prime}\right|=2$ and $P^{\prime \prime} \nsubseteq P \cup P^{\prime}$, then either $P$ is a triangle or $G^{\prime} \cong K_{4}$ :

Suppose that there exists such a $P^{\prime \prime}$.

First suppose that $P^{\prime \prime} \in \mathcal{P}^{*}$. Then, by Lemmas 4.15 and 4.16 , $P$ must be a triangle.

Next suppose that there is no such $P^{\prime \prime} \in \mathcal{P}^{*}$ and suppose that $P^{\prime \prime}$ is a triangle. The only way that we can have a triangle meeting with either $P$ or $P^{\prime}$ in two edges, is to have the situation shown below (remember that the graph is simple).



Note that the subgraph generated by $P \cup P^{\prime} \cup P^{\prime \prime} \cong K_{4}$. Suppose that $G^{\prime} \not K_{4}$. There there is some edge in $G^{\prime}$ not shown above and this edge is contained in an element of $\mathcal{P}$. However, this element of $\mathcal{P}$ must have size at least four, and must either intersect with $P$, or intersect with two edges of $P^{\prime}$; a contradiction to the assumption that there is no element of $\mathcal{P}^{*}$ that has an intersection of size two with $P$ or $P^{\prime}$. Therefore $G^{\prime} \cong K_{4}$.

Finally suppose that there is no such $P^{\prime \prime} \in \mathcal{P}^{*}$ and suppose that $P^{\prime \prime}$ is a three-element star. As $\left|P \cap P^{\prime \prime}\right|=2$ or $\left|P^{\prime} \cap P^{\prime \prime}\right|=2$, if $P$ is a star we must have the situation shown below:

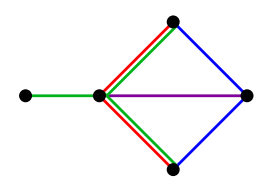

However, as $P^{\prime \prime} \in \mathcal{P}$, all three edges of $P$ must be in $P^{\prime \prime}$, and so $P^{\prime \prime} \in \mathcal{P}^{*}$. As $\left|P^{\prime \prime} \cap P\right|=2$ or $\left|P^{\prime \prime} \cap P^{\prime}\right|=2$ this is a contradiction. Therefore, if $P^{\prime \prime}$ is a three element star, $P$ must be a triangle.

Proof that, if no such $P^{\prime \prime}$ exists, then $P$ is a star or $G^{\prime} \cong K_{4}^{-}$:

Assume that there is for all $P^{\prime \prime} \nsubseteq P \cup P^{\prime}$ we have $\left|P \cap P^{\prime \prime}\right| \leq 1$ and $\left|P^{\prime} \cap P^{\prime \prime}\right| \leq$ 1. If $\left|P \cap P^{\prime \prime}\right|=0$ and $\left|P^{\prime} \cap P^{\prime \prime}\right|=0$ for all $P^{\prime \prime} \in \mathcal{P}$, then $G^{\prime} \cong K_{4}^{-}$. Therefore assume that $\left|P^{\prime \prime} \cap\left(P \cup P^{\prime}\right)\right| \geq 1$. 
If we can find some such $P^{\prime \prime} \in \mathcal{P}^{*}$, then, by Lemmas 4.15 and $4.16, P$ must be a star.

Therefore suppose that there is some $P^{\prime \prime}$ such that $\left|P^{\prime \prime} \cap\left(P \cup P^{\prime}\right)\right| \geq 1$, but no such $P^{\prime \prime} \in \mathcal{P}^{*}$. Suppose, for the purposes of contradiction, that $P$ is a triangle.

First assume that $P^{\prime \prime}$ is a triangle. If $P^{\prime \prime} \cap\left(P \cup P^{\prime}\right)=1$ then we must be in one of the situations shown below:
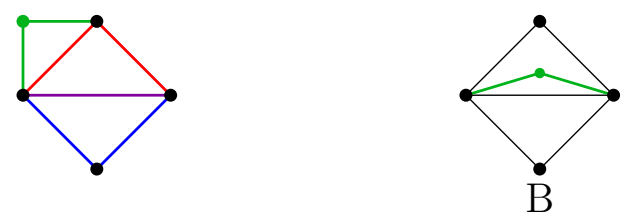

But in both these situations there is an intersection of a four element set and $P$, a contradiction.

If $P^{\prime \prime} \cap\left(P \cup P^{\prime}\right)=2$ then, as $P^{\prime \prime} \nsubseteq P \cup P^{\prime}$ and we are assuming that $P$ is a triangle, we must have the following situation:



But this gives a star that is not contained in $P \cup P^{\prime}$ that has an intersection of size two with $P$, a contradiction.

Next assume that $P^{\prime \prime}$ is a star and again suppose that $P$ is a triangle. There is no way to have $P$ intersecting $P^{\prime \prime}$ or $P^{\prime}$ intersecting $P^{\prime \prime}$ that does not given an intersection of size two between $P^{\prime \prime}$ and $P$ or $P^{\prime}$, a contradiction.

Therefore, if for all $P^{\prime \prime} \nsubseteq P \cup P^{\prime}$ we have $\left|P \cap P^{\prime \prime}\right| \leq 1$ or $\left|P^{\prime} \cap P^{\prime \prime}\right| \leq 1$, then either $G^{\prime} \cong K_{4}^{-}$or $P$ is a star.

We shall now assume, for the remainder of the section, that there is no element of $\mathcal{P}_{3}$ that has an intersection of size one with $P$.

As $\mathcal{A}$ is the collection of edge adjacencies of a graph, there can be no more than three members of $\mathcal{P}$ that have a 2 -element intersection with $P$.

Lemma 4.19. If there exists a 3-element set, $P^{\prime} \neq P$, that has a two element intersection with $P$, then one of $P, P^{\prime}$ is a 3-element star and the other is a 


\section{triangle}

Proof. If $P$ and $P^{\prime}$ were both triangles then $|V(P)|=\left|V\left(P^{\prime}\right)\right|=3$ and any two edges of $P$ must be incident with three vertices. As $\left|P \cap P^{\prime}\right|=2$ we have $\left|V\left(P \cap P^{\prime}\right)\right|=3$ and so there is no vertex of $V(P)$ or $V\left(P^{\prime}\right)$ that is not in $V\left(P \cap P^{\prime}\right)$. Therefore the edge of $P^{\prime}$ that is not in $P$ must be parallel to the edge of $P$ that is not in $P^{\prime}$ but this contradicts the fact that the graph is simple.

Suppose that both $P$ and $P^{\prime}$ were both stars and $P \cap P^{\prime}=\{e, f\}$ for some $e, f \in E(G)$. By the maximality of elements in $\mathcal{A}$, we know that $P$ and $P^{\prime}$ cannot share the same centre. Let $a$ be the centre of $P$. As $e \in P \cap P^{\prime}$, the centre of $P^{\prime}$ must be $V(\{e\})-\{a\}$. Similarly the centre of $P^{\prime}$ must also be $V(\{f\})-\{a\}$, and as $a \in(V(\{f\}) \cap V(\{e\}))$, we must have $V(\{e\})=V(\{f\})$ so $e$ and $f$ are parallel, a contradiction as our graph is simple.

Lemma 4.20. Suppose that there are two or more sets, $P^{\prime}$ and $P^{\prime \prime}$ from $\mathcal{P}_{3}$ that have a 2-element intersection with $P$. Then $P$ is a triangle.

Proof. Suppose that $P$ were a 3 -element star. Then $P^{\prime}$ and $P^{\prime \prime}$ would be triangles each with one edge not in $P$. This would mean that $P^{\prime}$ and $P^{\prime \prime}$ would have to share a common edge with each other and that edge must also be in $P$. This means that the edges of $P^{\prime}$ and $P^{\prime \prime}$ that are not in $P$ along with the edge of $P$ that is in both $P^{\prime}$ and $P^{\prime \prime}$ would form a three element star with a one element intersection with $P$; a contradiction, as we are assuming that there is no element of $\mathcal{P}_{3}$ that meets $P$ in a single edge.

Lemma 4.21. Suppose that there is exactly one set, $P^{\prime} \in \mathcal{P}_{3}$ that has a 2-element intersection with $P$. Then either the connected component of $G$ containing $P$ is isomorphic to $\bar{Y}$, or $P$ is a triangle.

Proof. Let $G^{\prime}$ be the connected component of $G$ that contains $P$. Suppose that $G^{\prime}$ is not isomorphic to $\bar{Y}$ and that $P$ is a star. Then we must have the situation below, where $P$ is shown in red and $P^{\prime}$ is shown in blue and the common edges are shown in purple:

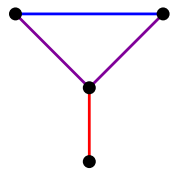


As $G^{\prime}$ is not isomorphic to $\bar{Y}$, then there is a set $Q$, in $\mathcal{P}$ that is not contained in $P \cup P^{\prime}$, but does share at least one edge with $P \cup P^{\prime}$. As there is no $P^{\prime} \in \mathcal{P}^{*}$ that intersects with $P$ in at least one element, and no member of $\mathcal{P}_{3}$ that is not $P$ or $Q$, that has $P^{\prime} \cap P \neq \emptyset$, the only way we can have this is to have $Q$ a triangle as shown below ( $Q$ is shown in green):



However, this gives a member of $\mathcal{P}-\left\{P \cup P^{\prime}\right\}$ (specifically the star with centre $v$ ) which has a 1-element intersection with $P$; a contradiction.

Lemma 4.22. Suppose that there is no member of $\mathcal{P}_{3}$ that has a non-empty intersection with $P$. Then $P$ is a connected component of $G$ and is either a triangle or a 3-element star.

This means that we have now identified which sets of $\mathcal{P}_{3}$ are 3 -element stars and which are triangles, except in some cases for small connected components. Our final step is to find all two element stars. The two element stars that are not part of a triangle are in $\mathcal{P}$ so we need to find out when a pair of elements of a triangle is a 2-element star.

Let the collection of all $P \in \mathcal{P}$ such that $P$ is not a triangle be known as $\mathcal{S}$.

Lemma 4.23. Let $P$ be a triangle and suppose that $P=\left\{e_{1}, e_{2}, e_{3}\right\}$. Then $\left\{e_{i}, e_{j}\right\}$, where $i, j \in\{1,2,3\}$ and $i \neq j$, is a two element star if, and only if, $\left\{e_{i}, e_{j}\right\} \nsubseteq S \in \mathcal{S}$.

Proof. As $e_{i}, e_{j}$ are adjacent either $\left\{e_{i}, e_{j}\right\}$ is a star or $\left\{e_{i}, e_{j}\right\}$ is properly contained in a star. If it is properly contained in a star then it will be a subset of an element of $\mathcal{S}$ as $\mathcal{S}$ is a complete collection of stars of more than two elements.

\section{Summary}

Given $\mathcal{P}$, to find the complete stars of $G$ we split $\mathcal{P}$ into two sets, $\mathcal{P}^{*}$ and $\mathcal{P}_{3}$, where $\mathcal{P}_{3}$ is the collection of all and only the three element members of $\mathcal{P}$ 
and $\mathcal{P}^{*}=\mathcal{P}-\mathcal{P}_{3}$. Every complete star that contains more than three edges is contained in $\mathcal{P}^{*}$ and these are the only sets of $\mathcal{P}^{*}$ except for some one and two element complete stars.

If a two element star is not contained in $\mathcal{P}^{*}$, then the edges in this star are two of the edges of a triangle.

Consider a member, $P$, of $\mathcal{P}_{3}$. To find out if $P$ is a three-element star:

i) Find a $P^{\prime} \in \mathcal{P}^{*}$ that has a non-empty intersection with $P$. If the intersection is of size one then $P$ is a star, if the intersection is of size two then $P$ is a triangle. The intersection cannot be of size greater than two.

ii) If no such $P^{\prime}$ exists find a $P^{\prime} \in \mathcal{P}_{3}$ such that $\left|P \cap P^{\prime}\right|=1$.

a) If there is some $e \in P$ such that for all $f \in P^{\prime}-P$ we have $\{e, f\} \notin \mathcal{A}$, then $P$ is a star.

b) If no such $e$ exists then:

- If there is $P^{\prime \prime} \nsubseteq P \cup P^{\prime}$ such that $P^{\prime \prime} \in \mathcal{P}$ and $\left|P \cap P^{\prime \prime}\right|=2$ or $\left|P^{\prime} \cap P^{\prime \prime}\right|=2$, then $P$ is a triangle or $G^{\prime} \cong K_{4}$.

- If there is no set $Q \nsubseteq P \cup P^{\prime} \cup P^{\prime \prime}$ such that $Q \in \mathcal{P}$ and $Q$ has non-empty intersection with $P \cup P^{\prime} \cup P^{\prime \prime}$, then $G^{\prime} \cong K_{4}$. Otherwise $P$ is a triangle.

- If there is no such $P^{\prime \prime}$ then either $G^{\prime} \cong K_{4}^{-}$or $P$ is a star.

- If there is no set $Q \nsubseteq P \cup P^{\prime}$ such that $Q \in \mathcal{P}$ and $Q$ has nonempty intersection with $P \cup P^{\prime}$, then $G^{\prime} \cong K_{4}^{-}$. Otherwise $P$ is a star.

iii) If there is no set in $\mathcal{P}_{3}$ that has an intersection of size one with $P$, then find $P^{\prime} \in \mathcal{P}_{3}$ such that $\left|P \cap P^{\prime}\right|=2$

(a) Suppose that there are two elements of $\mathcal{P}_{3}$ that have a two element intersection with $P$, then $P$ is a triangle.

(b) If there is exactly one member of $P^{\prime} \in \mathcal{P}_{3}$ that has a 2-element intersection with $P$, then $P$ is a triangle or $G^{\prime} \cong \bar{Y}$.

- If there is no member of $\mathcal{P}$ that is not contained in $P \cup P^{\prime}$ that has a non-empty intersection with $P$ or $P^{\prime}$, then $G \cong \bar{Y}$. Otherwise $P$ is a triangle. 
iv) If there no set in $\mathcal{P}_{3}$ that has an intersection of size one or two with $P$, then $G^{\prime} \cong K_{3}$ or $Y$.

We have now identified all stars of more than two elements. We have also identified all triangles. To find all two element stars we look at all pairs of edges $\{e, f\}$ that are in some triangle. If $\{e, f\} \nsubseteq P$ for any $P \in \mathcal{P}^{*}$, then $\{e, f\}$ is a two element star, otherwise it is not. The collection of all such pairs of edges along with the two element members of $\mathcal{P}^{*}$ gives the collection of all 2-element stars of the graph. To find all single elements stars we look at edges that appear in exactly one star. These, along with all sets of $\mathcal{P}^{*}$ of size one gives the collection of all single-element stars of the graph. This means that we are able, except in the ambiguous cases of $K_{3}, Y, \bar{Y}, K_{4}^{-}$and $K_{4}$, to identify all stars of the graph and therefore have a complete description of the graph. Given a list of stars of $G$ we can build $G$ from this list.

The algorithm also gives a way of identifying when we have a connected component isomorphic to $\bar{Y}$ or to $K_{4}^{-}$or to $K_{4}$ or when we have a connected component that is isomorphic to either $K_{3}$ or $Y$. The connectivity function cannot tell the difference between the following labellings of $K_{4}$.


so when, from the edge adjacencies, we identify that we have a connected component isomorphic to one of the above graphs, we may arbitrarily choose which one the graph contains. The other ambiguous cases can be told apart by their connectivity functions and will be dealt with shortly.

\subsection{When Is a Connectivity Function Graphic?}

In this section we shall give a method for establishing when a connectivity function, $\lambda$, is graphic. This algorithm uses a polynomial, in the size of the set on which the connectivity function is based, number of evaluations of 
the connectivity function. To do this we use the results from the previous sections to build a graph that would have connectivity function $\lambda$ were $\lambda$ graphic. We shall then show that if we have a connectivity function $\lambda$ and a graphic connectivity function, $\gamma$, both on set $E$, then if $\lambda$ and $\gamma$ agree on some particular subsets of $E$ then $\lambda$ and $\gamma$ will agree everywhere.

\section{Building a Graph From a Connectivity Function}

Suppose that we have a connectivity function $\lambda$ on $E$. We now give an algorithm that builds a graph which would have connectivity function $\lambda$ were $\lambda$ graphic. If $\lambda$ is not graphic we may still be able to build such a graph or at some stage of the algorithm we may be able to stop and state that $\lambda$ is not graphic. We will therefore assume that $\lambda$ is graphic and comes from graph $G$, and may at some stage find a contradiction to this.

From Section 4.1 we know that the leaves of $G$ are exactly those elements, $e \in E$ such that $\lambda(\{e\}) \leq 1$. If for any $a, b \in E$ we have $\lambda(\{a\})>2$ then we are able to say $\lambda$ is not graphic, otherwise Lemma 4.7 then gives a method for finding out which elements of $E$ are adjacent in $G$. The algorithm given for building a graph given the edge adjacencies requires the graph to be simple. We are assuming that $G$ does not contain any loops but $G$ may contain parallel edges. We shall therefore identify the parallel classes of $G$ using Lemmas 4.12 and 4.13 . Once we know the parallel classes we are able to find the adjacencies of an underlying simple graph, $G_{s}$, of $G$. We are then able to build $G_{s}$ using the algorithm given in Section 4.2. This will uniquely build $G_{s}$ in all but a few cases which we shall consider shortly. If there are more than three sets with a 2-element intersection with some element, $P$, of $\mathcal{P}_{3}$, then $\lambda$ is not graphic. Suppose that we have successfully found the collection of stars from the edge adjacencies. We must now check that this collection of stars is the collection of stars of a graph. To do this we must check that every element appears in no more than two stars. This will be so if, and only if, we have the collection of stars of a graph, and so if an element appears in more than two stars, then $\lambda$ is not graphic.

Having built $G_{s}$, we know which collections of edges form parallel classes in $G$. We are then able to add the parallel edges uniquely to $G_{s}$ to obtain $G$. In some cases $G_{s}$ may not be able to be uniquely built from the adja- 
cencies. However, the connectivity function gives more information than the adjacency relation, so in some of these cases we are able to build $G_{s}$ uniquely. The cases where we cannot build $G_{s}$ uniquely from the adjacency relation are when $G_{s}$ contains a connected component, $G_{s}^{\prime}$ isomorphic to one of $Y, K_{3}, \bar{Y}, K_{4}^{-}, K_{4}$. We now consider these cases. Note that we know all parallel classes of $G$.

Suppose that $G_{s}^{\prime}$ is isomorphic to $K_{3}$ or $Y$ (from the edge adjacency relation we cannot tell which). If there is some element, $e$, of $G_{s}^{\prime}$ such that $\lambda(\{e\})=1$ then this element is a leaf of $G_{s}$ (as $G_{s}$ contains no loops) and so $G_{s}^{\prime} \cong Y$. Suppose that there is no $e \in E\left(G_{s}^{\prime}\right)$ such that $\lambda(\{e\})=1$. Then either $G_{s}^{\prime} \cong K_{3}$, or $G_{s}^{\prime} \cong Y$ and for every $e \in E\left(G_{s}^{\prime}\right)$ there is some $f \in E(G)-E\left(G_{s}\right)$ such that $f$ is parallel to $e$. In this second case it must be that $\lambda\left(E\left(G_{s}^{\prime}\right)\right)=4$ as no vertex of $G_{s}^{\prime}$ is full in $G$ with respect to $G_{s}^{\prime}$. In the case where $G_{s}^{\prime} \cong K_{3}$ it must be that $\lambda\left(G_{s}^{\prime}\right) \leq 3$ as $G_{s}^{\prime}$ contains three vertices.

Suppose that $G_{s}^{\prime} \cong \bar{Y}$, the adjacency relation cannot tell the difference between the following two situations:



Suppose that we are in the first situation. Then $\lambda(\{d\})=1$ or else there is some edge of $G$ that is parallel to $d$. If there is some edge of $G$ that is parallel to $d$ then $\lambda(\{a, b, d\})=4$. This distinguishes this situation from the second one which has $\lambda(\{a, b, d\}) \leq 3$.

Suppose that $G_{s}^{\prime} \cong K_{4}^{-}$. Then from the edge adjacencies we cannot distinguish between the following two graphs:

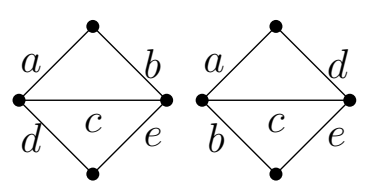

Either there is no edge parallel to $a$ or $b$ in which case $\lambda(\{a, b, c\})=2$ in the first case and $\lambda(\{a, b, c\}) \geq 3$ in the second case, or there is an edge parallel to at least one of $a$ or $b$. Without loss of generality say that there is an edge 
parallel to $a$, then $\lambda(\{a, b, c\})=3$ in the first case and $\lambda(\{a, b, c\})=4$ in the second case.

Suppose that $G_{s}^{\prime} \cong K_{4}$. From the edge adjacencies we cannot distinguish between the following two graphs:

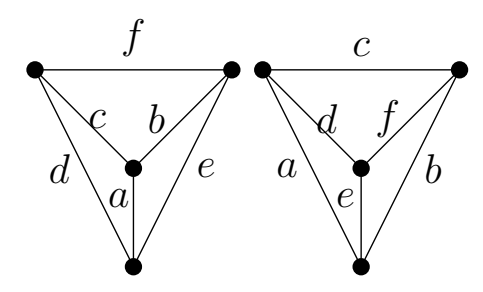

If there are no edges of $G$ parallel to edges of $G_{s}^{\prime}$, the connectivity function of the two connected components are the same so we cannot distinguish between the two cases. Suppose that there is an edge of $G$ parallel to $a$. In the first case this gives $\lambda(\{a, d, e\})=4$ so we know that $\{a, d, e\}$ is a star and in the second case $\lambda(\{a, d, e\})=3$ so $\{a, d, e\}$ is a triangle. Once we know one star or triangle of $K_{4}$ we can find the rest of the stars and triangles and distinguish between stars and triangles that are subgraphs induced by three element subsets of $K_{4}$.

Therefore except when $G$ has a connected component isomorphic to $K_{4}$ we can build $G$ uniquely (except that we cannot distinguish loops from leaves) from its connectivity function.

\section{When is a Connectivity function Equal to a Graphic Connectivity Function?}

The next question is, given we have been able to build this graph, is $\lambda$ necessarily graphic. The answer is no, we cannot say that $\lambda$ is graphic just because we have built the graph that would have $\lambda$ as its connectivity function were $\lambda$ graphic. For consider the following two connectivity functions $\lambda$ and $\gamma$ described on the next page: 


\begin{tabular}{|l|l|l|}
\hline & $\lambda$ & $\gamma$ \\
\hline$\{\mathrm{a}\}$ & 2 & 2 \\
\hline$\{\mathrm{b}\}$ & 2 & 2 \\
\hline$\{\mathrm{c}\}$ & 2 & 2 \\
\hline$\{\mathrm{d}\}$ & 2 & 2 \\
\hline$\{\mathrm{e}\}$ & 2 & 2 \\
\hline$\{\mathrm{f}\}$ & 2 & 2 \\
\hline$\{\mathrm{a}, \mathrm{b}\}$ & 3 & 3 \\
\hline$\{\mathrm{a}, \mathrm{c}\}$ & 3 & 3 \\
\hline$\{\mathrm{a}, \mathrm{d}\}$ & 3 & 3 \\
\hline$\{\mathrm{a}, \mathrm{e}\}$ & 3 & 3 \\
\hline$\{\mathrm{a}, \mathrm{f}\}$ & 4 & 4 \\
\hline$\{\mathrm{b}, \mathrm{c}\}$ & 3 & 3 \\
\hline$\{\mathrm{b}, \mathrm{d}\}$ & 4 & 4 \\
\hline$\{\mathrm{b}, \mathrm{e}\}$ & 3 & 3 \\
\hline$\{\mathrm{b}, \mathrm{f}\}$ & 3 & 3 \\
\hline$\{\mathrm{c}, \mathrm{d}\}$ & 3 & 3 \\
\hline$\{\mathrm{c}, \mathrm{e}\}$ & 4 & 4 \\
\hline$\{\mathrm{c}, \mathrm{f}\}$ & 3 & 3 \\
\hline$\{\mathrm{d}, \mathrm{e}\}$ & 3 & 3 \\
\hline$\{\mathrm{d}, \mathrm{f}\}$ & 3 & 3 \\
\hline$\{\mathrm{e}, \mathrm{f}\}$ & 3 & 3 \\
\hline$\{\mathrm{a}, \mathrm{b}, \mathrm{c}\}$ & 4 & 3 \\
\hline$\{\mathrm{a}, \mathrm{b}, \mathrm{d}\}$ & 4 & 4 \\
\hline$\{\mathrm{a}, \mathrm{b}, \mathrm{e}\}$ & 3 & 3 \\
\hline$\{\mathrm{a}, \mathrm{b}, \mathrm{f}\}$ & 4 & 4 \\
\hline$\{\mathrm{a}, \mathrm{c}, \mathrm{d}\}$ & 3 & 3 \\
\hline$\{\mathrm{a}, \mathrm{c}, \mathrm{e}\}$ & 4 & 4 \\
\hline$\{\mathrm{a}, \mathrm{c}, \mathrm{f}\}$ & 4 & 4 \\
\hline$\{\mathrm{a}, \mathrm{d}, \mathrm{e}\}$ & 3 & 3 \\
\hline$\{\mathrm{a}, \mathrm{d}, \mathrm{f}\}$ & 4 & 4 \\
\hline$\{\mathrm{a}, \mathrm{e}, \mathrm{f}\}$ & 4 & 4 \\
\hline & & \\
\hline
\end{tabular}

The remaining values can be found by symmetry. Note that $\lambda \neq \gamma$ as $\lambda(\{a, b, c\})=4$ and $\gamma(\{a, b, c\})=3$. 
If we consider the edge adjacencies of the graph that would have $\lambda$ as its connectivity function were $\lambda$ graphic, we find that this graph would have to be isomorphic to one of the graphs shown below, and to establish this we only need to check sets of size at most two.

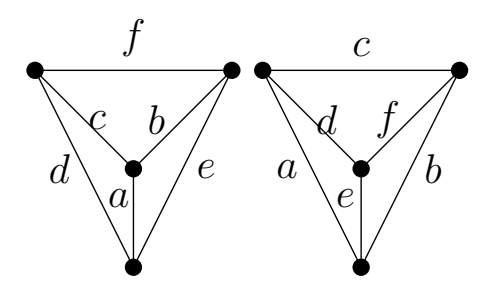

However, these graphs have connectivity function, $\gamma$, Therefore $\lambda \neq \gamma$ so $\lambda$ cannot be a graphic connectivity function despite the fact that we were able to build a graph that would have $\lambda$ as its connectivity function were $\lambda$ graphic.

We now prove that, if $\lambda$ and $\gamma$ agree on some particular collection of sets, then $\lambda$ and $\gamma$ agree everywhere and so $\lambda$ is graphic.

Definition 4.24. If $S$ is a complete star of $G$ and $e \in S$ then $S-\{e\}$ is an almost-complete star of $G$. Let $S$ is a complete star of $G$, and $e \in S$. For any $a \in E$ that is adjacent to $e$ but not in $S$, the set $(S-\{e\}) \cup\{a\}$ is a broken star of $G$.

The definition of a broken star is a little hard to digest so a couple of pictures of broken stars are given below, where the dashed red line is in $G$ but not in the broken star.
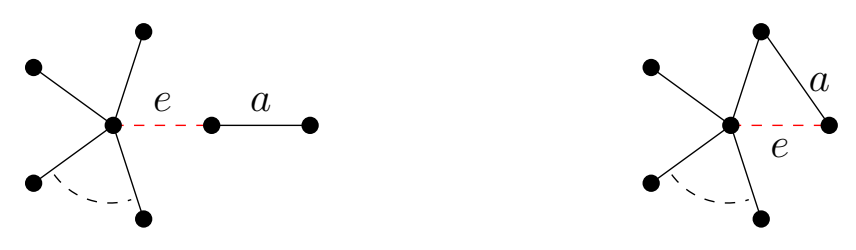

Recall that a parallel class is a maximal parallel set. In this section we prove the following theorem:

Theorem 4.25. Let $G$ be a graph with connectivity function $\gamma$. Let $\lambda$ be a connectivity function on $E(G)$ such that $\lambda(X)=\gamma(X)$ whenever $X \subseteq E$ satisfies any one of the following: 
$C 1|X| \leq 2$

C2 $X$ is a complete star of $G$,

C3 $X$ is an almost-complete star of $G$,

$C_{4} X$ is a broken star of $G$,

$C 5 X$ is a triangle, maximal parallel triangle, or $X \cup\{e\}$ is a maximal parallel triangle for some $e \in E(G)-E(X)$,

C6 $X$ is a parallel class in $G$.

Then $\lambda(X)=\gamma(X)$, for all $X \subseteq E$.

Suppose that we are given an arbitrary connectivity function, $\lambda$, on a set $E$. We can then build a graph, $G$, in the way described earlier in the section (labelled Building a Graph From a Connectivity Function). Now consider the connectivity function, $\gamma$ of $G$. Suppose that, for all $X \subseteq E$ such that one of $C 1-C 6$ from Theorem 4.25 holds, $\gamma(X)=\lambda(X)$. Then, by Theorem 4.25 . $\lambda(X)=\gamma(X)$ for all $X \subseteq E$, and so $\lambda$ is a graphic connectivity function. This means that, given Theorem 4.25, we have proved Theorem 4.1 holds, in other words we have proved the following theorem:

Theorem 4.1. There is some polynomial $p$ such that, given an arbitrary connectivity function $\lambda$, we are able to establish whether or not $\lambda$ is graphic in at most $p(n)$ evaluations of the connectivity function, where $n$ is the size of the set on which $\lambda$ is based.

The following lemma is immediate from the definition of graphic connectivity functions.

Lemma 4.26. Suppose that $G$ is a graph with connectivity function $\gamma$. Then $\gamma(X)$ is the number of non-full vertices of $G$ with respect to $X$.

If $S$ is a star of $G$ then we shall use $\widetilde{S}$ to denote the complete star of $G$ containing $S$; and if $P$ is a parallel set of $G$ then we shall use $\dddot{P}$ to denote the parallel class containing $P$ in $G$.

Throughout this section we shall consider $G$ to be a loopless graph with no isolated vertices, whose edge set is $E$ whose connectivity function is $\gamma$. Let 
$\lambda$ be a connectivity function on $E$ such that $\lambda(X)=\gamma(X)$ if one of $C 1$-C6 from Theorem 4.25 holds.

We will define a function full $v_{v_{i}}: 2^{E} \rightarrow\{0,1\}$ by

$$
\text { full }_{v_{i}}(X)= \begin{cases}1 & \text { if } v_{i} \text { is full in } G \text { with respect to X } \\ 0 & \text { otherwise }\end{cases}
$$

for all $X \subseteq 2^{E}$.

Lemma 4.27. Let $P$ be a parallel set in $G$. Then $\lambda(P)=\gamma(P)$.

Proof. Let $V(P)=\left\{v_{1}, v_{2}\right\}$. Suppose that $v_{1}$ or $v_{2}$ is full with respect to $P$. Then $P$ is a complete star of $G$ and so $\lambda(P)=\gamma(P)$ by $C 2$. We shall therefore assume that neither $v_{1}$ nor $v_{2}$ is full in $G$ with respect to $P$.

First, we show that $\lambda(P) \leq \gamma(P)$. If $|P| \leq 2$ then this result holds by $C 1$ so we assume that $|P| \geq 3$. Assume for the purposes of induction that this result holds for all parallel sets $P^{\prime}$ such that $\left|P^{\prime}\right|<|P|$. Let $e \in P$. As $P$ is not a complete star of $G$ and $|P| \geq 3$, we have $\gamma(P-\{e\})=\gamma(P)$ and for $f \in P-\{e\}$ we have $\gamma(\{e, f\})=\gamma(\{f\})$. Therefore:

$$
\begin{aligned}
\gamma(P)+\gamma(\{f\}) & =\gamma(P-\{e\})+\gamma(\{e, f\}) \\
& \geq \lambda(P-\{e\})+\lambda(\{e, f\}) \\
& \geq \lambda(P)+\lambda(\{f\}) \\
& =\lambda(P)+\gamma(\{f\}),
\end{aligned}
$$

where (2) follows from (1) by the induction hypothesis and $C 1,(3)$ follows from (2) by submodularity, and (4) follows from (3) by $C 1$.

Therefore $\lambda(P) \leq \gamma(P)$.

Next, we show that $\lambda(P) \geq \gamma(P)$. We do this by induction on $|\dddot{P}-P|$. If $|\dddot{P}-P|=0$, then $P$ is a parallel class, so the result holds by $C 6$. Assume that the result holds for all $P^{\prime} \subseteq \dddot{P}$ such that $\left|\dddot{P}-P^{\prime}\right|<k$ and suppose that $|\dddot{P}-P|=k$ for some $k \geq 1$. Let $e \in P$ and $f \in \dddot{P}-P$. If neither vertex of $P \cup\{f\}$ is full in $G$ with respect to $P \cup\{f\}$ then $\gamma(P)=\gamma(P \cup\{f\})$ and 
$\gamma(\{e, f\})=\gamma(\{e\})$. Therefore

$$
\begin{aligned}
\gamma(P)+\gamma(\{e, f\}) & =\gamma(P \cup\{f\})+\gamma(\{e\}) \\
& \leq \lambda(P \cup\{f\})+\lambda(\{e\}) \\
& \leq \lambda(P)+\lambda(\{e, f\}) \\
& =\lambda(P)+\gamma(\{e, f\}),
\end{aligned}
$$

where (2) follows from (1) by the induction hypothesis and $C 1$, (3) follows from (2) by submodularity, and (4) follows from (3) by $C 1$. Therefore, in this case, $\lambda(P) \geq \gamma(P)$.

If some vertex of $P \cup f$ were full in $G$ with respect to $P \cup f$, then $P$ would be an almost-complete star of $G$, so $\lambda(P)=\gamma(P)$ by $C 3$.

Therefore $\lambda(P) \geq \gamma(P)$

Lemma 4.28. Suppose that $S$ is a star of $G$ with centre $v$. Then $\lambda(S)=$ $\gamma(S)$.

Proof. We shall assume that $S \neq \emptyset$ and $S \neq \widetilde{S}$, as in these cases the result follows directly from $C 2$.

First we show that $\lambda(S) \geq \gamma(S)$. We do this by induction on $|\widetilde{S}-S|$. If $|\widetilde{S}-S| \leq 1$, then the result holds by $C 3$. For the purposes of induction we assume that this result holds for all $S^{\prime} \subseteq \widetilde{S}$ such that $\left|\widetilde{S}-S^{\prime}\right|<k$. Let $|\widetilde{S}-S|=k$ for $k>1$, and let $a \in \widetilde{S}-S$ be such that $V(\{a\})=\left\{v, v_{1}\right\}$. Let $e \in S$.

Suppose that $a$ is not parallel to any edge of $S$. As $|\widetilde{S}-S|>1$, we have $\gamma(S)=\gamma(S \cup\{a\})-\left(1-\right.$ full $\left._{v_{1}}(\{a\})\right)$ and $\gamma(\{e, a\})=\gamma(\{e\})+\left(1-\right.$ full $\left._{v_{1}}(\{a\})\right)$.

Therefore:

$$
\begin{aligned}
\gamma(S)+\gamma(\{a, e\}) & =\gamma(S \cup\{a\})+\gamma(\{e\}) \\
& \leq \lambda(S \cup\{a\})+\lambda(\{e\}) \\
& \leq \lambda(S)+\lambda(\{a, e\}) \\
& =\lambda(S)+\gamma(\{a, e\}),
\end{aligned}
$$

where (2) follows from (1) by the induction hypothesis and $C 1,(3)$ follows from (2) by submodularity and (4) follows from (3) by $C 1$. Therefore, in this 
case, $\gamma(S) \leq \lambda(S)$.

Now suppose that $a$ is parallel to some edge of $S$ and let $P$ be the maximal parallel subset of $S-\{a\}$ with which $a$ is parallel. Then $\gamma(S)=\gamma(S \cup\{a\})+$ full $_{v_{1}}(S \cup\{a\})$ and $\gamma(P \cup\{a\})=\gamma(P)-$ full $_{v_{1}}(P \cup\{a\})$, as $|\widetilde{S}-S|>1$. As full $_{v_{1}}(S \cup\{a\})=$ full $_{v_{1}}(P \cup\{a\})$ we have:

$$
\begin{aligned}
\gamma(S)+\gamma(P \cup\{a\}) & =\gamma(S \cup\{a\})+\gamma(P) \\
& \leq \lambda(S \cup\{a\})+\lambda(P) \\
& \leq \lambda(S)+\lambda(P \cup\{a\}) \\
& =\lambda(S)+\gamma(P \cup\{a\}),
\end{aligned}
$$

where (2) follows from (1) by the induction hypothesis and Lemma 4.27, (3) follows from (2) by submodularity, and (4) follows from (3) by Lemma 4.27 . Therefore $\lambda(S) \geq \gamma(S)$.

Next we show that $\lambda(S) \leq \gamma(S)$. We shall do this by induction on the number of edges contained in non-trivial parallel classes of $S$. The following claim provides our base case:

Claim 4.28.1. If $S$ contains no parallel edges than $\lambda(S) \leq \gamma(S)$

Proof. The result holds for $S$ such that $|S| \leq 2$ by $C 1$. For the purposes of induction suppose that the result holds for all $S$ such that $|S|<m$.

Let $S=\left\{b_{1}, \ldots, b_{m}\right\}$, for some $m \geq 3$, and consider the subsets, $\left\{b_{1}, b_{2}\right\}$ and $\left\{b_{2}, \ldots, b_{m}\right\}$, of $S$. Let $V\left(\left\{b_{1}\right\}\right)=v, v_{1}$. As $\left\{b_{1}, \ldots, b_{m}\right\}$ is not a complete star of $G, \gamma\left(\left\{b_{1}, b_{2}\right\}\right)=\gamma\left(\left\{b_{2}\right\}\right)+\left(1-\right.$ full $\left._{v_{1}}\left(\left\{b_{1}\right\}\right)\right)$ and $\gamma\left(\left\{b_{2}, \ldots, b_{m}\right\}\right)=\gamma\left(\left\{b_{1}, \ldots, b_{m}\right\}\right)-\left(1-\right.$ full $\left._{v_{1}}\left(b_{1}\right)\right)$ Therefore:

$$
\begin{aligned}
\gamma\left(\left\{b_{1}, \ldots, b_{m}\right\}\right)+\gamma\left(\left\{b_{2}\right\}\right) & =\gamma\left(\left\{b_{2}, \ldots, b_{m}\right\}\right)+\gamma\left(\left\{b_{1}, b_{2}\right\}\right) \\
& \geq \lambda\left(\left\{b_{2}, \ldots, b_{m}\right\}\right)+\lambda\left(\left\{b_{1}, b_{2}\right\}\right) \\
& \geq \lambda\left(\left\{b_{1}, \ldots, b_{m}\right\}\right)+\lambda\left(\left\{b_{2}\right\}\right) \\
& =\lambda\left(\left\{b_{1}, \ldots, b_{m}\right\}\right)+\gamma\left(\left\{b_{2}\right\}\right),
\end{aligned}
$$

where (2) follows from (1) by $C 1$ and the induction hypothesis of the claim, (3) follows from (2) by submodularity, and (4) follows from (3) 
by $C 1$. Therefore $\lambda(S) \leq \gamma(S)$ when $S$ contains no parallel edges.

Assume, for the purposes of induction, that the result holds for all stars $S^{\prime}$ with less than $k$ edges contained in non-trivial parallel subsets of $S$. Let $S$ be a star with $k>1$ edges contained in non-trivial parallel subsets of $S$. Let $b_{1}$ be an edge of one such parallel set, which we shall call $P_{b_{1}}$. Either $S=P_{b_{1}}$ or there is some edge, $e$, which is not contained in $P_{b_{1}}$ but is contained in $S$. If $S=P_{b_{1}}$, then the result holds by Lemma 4.27; so suppose that there exists $e \in S-P_{b_{1}}$. Let $v_{1}$ be the vertex of $G$ that is incident with $b_{1}$ but not with $e$. Then $\gamma\left(S-\left\{b_{1}\right\}\right)=\gamma(S)+$ full $_{v_{1}}\left(P_{b_{1}}\right)$ and $\gamma\left(P_{b_{1}}-\left\{b_{1}\right\}\right)=\gamma\left(P_{b_{1}}\right)+$ full $_{v_{1}}\left(P_{b_{1}}\right)$. Therefore:

$$
\begin{aligned}
\gamma(S)+\gamma\left(P_{b_{1}}-\left\{b_{1}\right\}\right) & =\gamma\left(S-\left\{b_{1}\right\}\right)+\gamma\left(P_{b_{1}}\right) \\
& \geq \lambda\left(S-\left\{b_{1}\right\}\right)+\lambda\left(P_{b_{1}}\right) \\
& \geq \lambda(S)+\lambda\left(P_{b_{1}}-\left\{b_{1}\right\}\right) \\
& =\lambda(S)+\gamma\left(P_{b_{1}}-\left\{b_{1}\right\}\right),
\end{aligned}
$$

where (2) follows from (1) by the induction hypothesis and Lemma 4.27, (3) follows from (2) by submodularity, and (4) follows from (3) by Lemma 4.27 . Therefore $\lambda(S) \leq \gamma(S)$

Lemma 4.29. Let $P_{1}, P_{2}, P_{3}$ be three parallel sets of $G$ such that $P_{1} \cup P_{2} \cup P_{3}$ is a parallel triangle in $G$. Then $\lambda\left(P_{1} \cup P_{2} \cup P_{3}\right)=\gamma\left(P_{1} \cup P_{2} \cup P_{3}\right)$.

Proof. Let $V\left(P_{1}\right)=\left\{v_{1}, v_{2}\right\}, V\left(P_{2}\right)=\left\{v_{2}, v_{3}\right\}$ and $V\left(P_{3}\right)=\left\{v_{3}, v_{1}\right\}$ We shall first show, by induction, that $\lambda\left(P_{1} \cup P_{2} \cup P_{3}\right) \geq \gamma\left(P_{1} \cup P_{2} \cup P_{3}\right)$. If $\left|\left(\dddot{P}_{1} \cup \dddot{P}_{2} \cup \dddot{P}_{3}\right)-\left(P_{1} \cup P_{2} \cup P_{2}\right)\right| \leq 1$ then the result holds by $C 5$. Therefore we shall assume that $\left|\left(\dddot{P}_{1} \cup \dddot{P}_{2} \cup \dddot{P}_{3}\right)-\left(P_{1} \cup P_{2} \cup P_{2}\right)\right| \geq 2$ and without loss of generality let $e \in \dddot{P}_{1}-P_{1}$. As $\left(P_{1} \cup\{e\}\right) \cup P_{2} \cup P_{3}$ is not a maximal parallel triangle it must contain at most one full vertex. If $v_{1}$ is not full with respect to $\left(P_{1} \cup\{e\}\right) \cup P_{2} \cup P_{3}$, then $\gamma\left(P_{1} \cup P_{2}\right)=\gamma\left(P_{1} \cup\{e\} \cup P_{2}\right)+$ full $_{v_{2}}\left(P_{1} \cup\{e\} \cup P_{2}\right)$ and $\gamma\left(P_{1} \cup P_{2} \cup P_{3}\right)=\gamma\left(\left(P_{1} \cup\{e\}\right) \cup P_{2} \cup P_{3}\right)+$ full $_{v_{2}}\left(\left(P_{1} \cup\{e\}\right) \cup P_{2} \cup P_{3}\right)$. 
As full $v_{2}\left(\left(P_{1} \cup\{e\}\right) \cup P_{2} \cup P_{3}\right)=$ full $_{v_{2}}\left(P_{1} \cup\{e\} \cup P_{2}\right)$ we have:

$$
\begin{aligned}
\gamma & \left(P_{1} \cup P_{2} \cup P_{3}\right)+\gamma\left(P_{1} \cup\{e\} \cup P_{2}\right) \\
& =\gamma\left(\left(P_{1} \cup\{e\}\right) \cup P_{2} \cup P_{3}\right)+\gamma\left(P_{1} \cup P_{2}\right) \\
& \leq \lambda\left(\left(P_{1} \cup\{e\}\right) \cup P_{2} \cup P_{3}\right)+\lambda\left(P_{1} \cup P_{2}\right) \\
& \leq \lambda\left(P_{1} \cup P_{2} \cup P_{3}\right)+\lambda\left(P_{1} \cup\{e\} \cup P_{2}\right) \\
& =\lambda\left(P_{1} \cup P_{2} \cup P_{3}\right)+\gamma\left(P_{1} \cup\{e\} \cup P_{2}\right)
\end{aligned}
$$

where (3) follows from (2) by the induction hypothesis and Lemma 4.28, (4) follows from (3) by submodularity and (5) follows from (4) by Lemma 4.28. If $v_{1}$ were full with respect to $P_{1} \cup P_{2} \cup P_{3}$, then $v_{2}$ would not be and so we could use a similar argument using the pair $P_{1}, P_{3}$ instead of $P_{1}, P_{2}$. Therefore $\gamma\left(P_{1} \cup P_{2} \cup P_{3}\right) \leq \lambda\left(P_{1} \cup P_{2} \cup P_{3}\right)$.

We now show that $\lambda\left(P_{1} \cup P_{2} \cup P_{3}\right) \leq \gamma\left(P_{1} \cup P_{2} \cup P_{3}\right)$. To do this we first need to note that the result holds if $P_{1} \cup P_{2} \cup P_{3}$ is a maximal parallel triangle of $G$ by $C 5$, and if $P_{1} \cup P_{2} \cup P_{3}$ is not a maximal parallel triangle of $G$ it must contain at least two non-full vertices. Also note that the result holds for triangles by $C 5$. Assume for the purposes of induction that the result holds for all parallel triangles that are strict subsets of $P_{1} \cup P_{2} \cup P_{3}$ and assume that $P_{1} \cup P_{2} \cup P_{3}$ is not a maximal parallel triangle.

Suppose that $\left|P_{1}\right| \geq 2$ and $e \in P_{1}$. As $P_{1} \cup P_{2} \cup P_{2}$ is not maximal, at most one of $v_{1}$ and $v_{2}$ can be full with respect to $P_{1} \cup P_{2} \cup P_{3}$. Without loss of generality we shall assume that $v_{1}$ is not full with respect to $P_{1} \cup P_{2} \cup P_{3}$. Note that $\gamma\left(\left(P_{1}-\{e\}\right) \cup P_{2} \cup P_{3}\right)=\gamma\left(P_{1} \cup P_{2} \cup P_{3}\right)+$ full $_{v_{2}}\left(P_{1} \cup P_{2} \cup P_{3}\right)$ and $\gamma\left(P_{1} \cup P_{2}\right)=\gamma\left(\left(P_{1}-\{e\}\right) \cup P_{2}\right)-$ full $_{v_{2}}\left(P_{1} \cup P_{2}\right)$. Therefore, as full $v_{2}\left(P_{1} \cup\right.$ $\left.P_{2} \cup P_{3}\right)=$ full $_{v_{2}}\left(P_{1} \cup P_{2}\right)$ we have $\gamma\left(\left(P_{1}-\{e\}\right) \cup\left(P_{2} \cup P_{3}\right)+\gamma\left(P_{1} \cup P_{2}\right)=\right.$ $\gamma\left(P_{1} \cup P_{2} \cup P_{3}\right)+\gamma\left(\left(P_{1}-\{e\}\right) \cup P_{2}\right)$. Therefore:

$$
\begin{aligned}
& \gamma\left(P_{1} \cup P_{2} \cup P_{3}\right)+\gamma\left(\left(P_{1}-\{e\}\right) \cup P_{2}\right) \\
& \quad=\gamma\left(\left(P_{1}-\{e\}\right) \cup P_{2} \cup P_{3}\right)+\gamma\left(P_{1} \cup P_{2}\right) \\
& \quad \geq \lambda\left(\left(P_{1}-\{e\}\right) \cup P_{2} \cup P_{3}\right)+\lambda\left(P_{1} \cup P_{2}\right) \\
& \quad \geq \lambda\left(P_{1} \cup P_{2} \cup P_{3}\right)+\lambda\left(\left(P_{1}-\{e\}\right) \cup P_{2}\right) \\
& \quad=\lambda\left(P_{1} \cup P_{2} \cup P_{3}\right)+\gamma\left(\left(P_{1}-\{e\}\right) \cup P_{2}\right),
\end{aligned}
$$


where (3) follows from (2) by the induction hypothesis and Lemma 4.28, (4) follows from (3) by submodularity and (5) follows from (4) by Lemma 4.28 .

Lemma 4.30. Let $S$ be a partial star of $G$ with centre $v$, and $P$ a parallel set of $G$ which is adjacent to an edge $f$ of $S$. Then $\lambda(S \cup P) \leq \gamma(S \cup P)$.

Proof. If $P \subseteq \widetilde{S}$ then we are done by Lemma 4.28, so we will assume that $P$ is not contained in $\widetilde{S}$.

Suppose that $P$ does not form a parallel triangle with any pair of edges of $S$. Let $V(P)=\left\{v_{1}, v_{2}\right\}$ and let $f \in S$ be such that $V(\{f\})=\left\{v_{1}, v\right\}$. We shall use $P_{f}$ to denote the collection of edges of $S$ that are parallel to $f$.

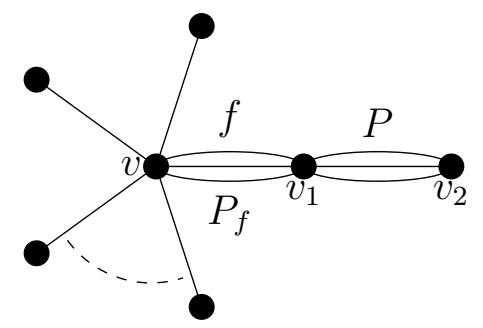

Note that $\gamma(S \cup P)=\gamma(S)+\left(1-\right.$ full $\left._{v_{2}}(P)\right)-$ full $_{v_{1}}\left(P \cup P_{f}\right)$ and $\gamma\left(P \cup P_{f}\right)=$ $\gamma\left(P_{f}\right)+\left(1-\right.$ full $\left._{v_{2}}(P)\right)-$ full $_{v_{1}}\left(P \cup P_{f}\right)$. Therefore:

$$
\begin{aligned}
\gamma(S \cup P)+\gamma\left(P_{f}\right) & =\gamma(S)+\gamma\left(P \cup P_{f}\right) \\
& =\lambda(S)+\lambda\left(P \cup P_{f}\right) \\
& \geq \lambda(S \cup P)+\lambda\left(P_{f}\right) \\
& =\lambda(S \cup P)+\gamma\left(P_{f}\right),
\end{aligned}
$$

where (2) follows from (1) by Lemma 4.28, (3) follows from (2) by submodularity and (4) follows from (3) by Lemma 4.28. Therefore $\lambda(S \cup P) \leq \gamma(S \cup P)$ when $P$ does not form a parallel triangle with any edges of $S$.

Now suppose that $P$ forms a parallel triangle with two classes of parallel edges of $S$ which we shall call $Q_{1}$ and $Q_{2}$. Let $V(P)=\left\{v_{1}, v_{2}\right\}, V\left(Q_{1}\right)=\left\{v_{1}, v\right\}$ and $V\left(Q_{2}\right)=\left\{v_{2}, v\right\}$. 




Then $\gamma(S)=\gamma(S \cup P)+$ full $_{v_{1}}\left(P \cup Q_{1}\right)+$ full $_{v_{2}}\left(P \cup Q_{2}\right)$ and $\gamma\left(P \cup Q_{1} \cup Q_{2}\right)=$ $\gamma\left(Q_{1} \cup Q_{2}\right)-$ full $_{v_{1}}\left(P \cup Q_{1}\right)-$ full $_{v_{2}}\left(P \cup Q_{2}\right)$. Therefore:

$$
\begin{aligned}
\gamma(S \cup P)+\gamma\left(Q_{1} \cup Q_{2}\right) & =\gamma(S)+\gamma\left(Q_{1} \cup Q_{2} \cup P\right) \\
& =\lambda(S)+\lambda\left(Q_{1} \cup Q_{2} \cup P\right) \\
& \geq \lambda(S \cup P)+\lambda\left(Q_{1} \cup Q_{2}\right) \\
& =\lambda(S \cup P)+\gamma\left(Q_{1} \cup Q_{2}\right),
\end{aligned}
$$

where (2) follows from (1) by Lemmas 4.28 and 4.29, (3) follows from (2) by submodularity and (4) follows from (3) by Lemma 4.28.

Therefore $\lambda(S \cup P) \leq \gamma(S \cup P)$

Lemma 4.31. Let $S$ be a partial star of $G$ with centre $v$, and e an element of $E(G)-S$ which is adjacent to an edge, a, of $S$. Then $\lambda((S \cup\{e\})-\{a\})=$ $\gamma((S \cup\{e\})-\{a\})$.

Proof. If $e$ is contained in the complete star containing $S$ then the result holds by Lemma 4.28. Therefore, for the remainder of the proof, we shall assume that $e$ is not contained in the complete star containing $S$. This means that the sets we are interested in have one of the forms shown below, where the dotted lines indicate some of the edges of $G$ that are not in $(S \cup\{e\})-\{a\}$.
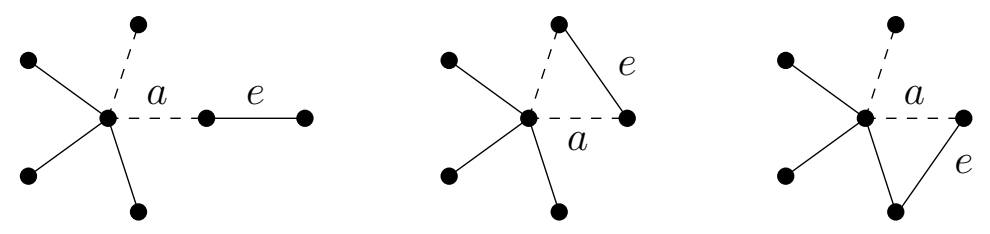

First we show that $\lambda(S \cup\{e\}-\{a\}) \leq \gamma((S \cup\{e\})-\{a\})$. If $a$ is parallel to some other element of $S$ or $e$ forms a triangle with some elements of $S$, then the result holds by Lemma 4.30 To do this, note that $\gamma(S-\{a\})+\gamma(\{e\})=$ 
$\gamma((S \cup\{e\})-\{a\})$. Therefore:

$$
\begin{aligned}
\gamma(S \cup\{e\}-\{a\}) & =\gamma(S-\{a\})+\gamma(\{e\}) \\
& =\lambda(S-\{a\})+\lambda(\{e\}) \\
& \geq \lambda((S \cup\{e\})-\{a\}),
\end{aligned}
$$

where (2) comes from (1) by Lemma 4.28 and $C 1$, and (3) comes from (2) by submodularity.

Next we show that $\lambda((S \cup\{e\})-\{a\}) \geq \gamma((S \cup\{e\})-\{a\})$. We do this by induction on $|\widetilde{S}-S|$. By $C 4$ the result holds when $|\widetilde{S}-S|=0$. Assume that the result holds for $S$ such that $|\widetilde{S}-S|<k$. Suppose that $|\widetilde{S}-S|=k$, where $k \geq 1$, and let $f^{\prime}$ be an edge of $S$ that is not $a$ and that is not adjacent to $e$, (if we cannot find such an edge then $|S \cup\{e\}-\{a\}| \leq 2$ so the result holds by $C 1)$. Let $f \in \widetilde{S}-S$ be such that $V(f)=\left\{v, v_{1}\right\}$.

Suppose that $f$ is not parallel to any element of $S$.

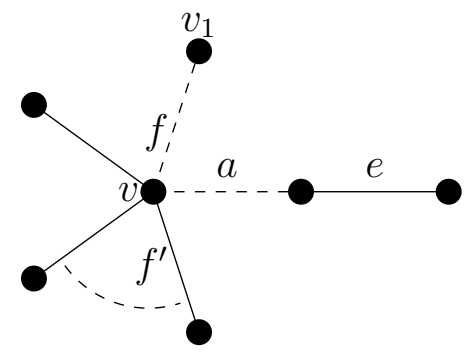

Case 1

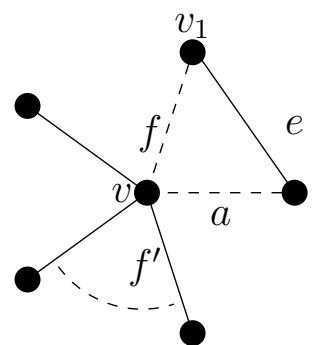

Case 2

In Case 1, $\gamma((S \cup\{e\})-\{a\})=\gamma((S \cup\{e, f\})-\{a\})-\left(1-\right.$ full $\left._{v_{1}}(f)\right)$ and $\gamma\left(\left\{f, f^{\prime}, e\right\}\right)=\gamma\left(\left\{f^{\prime}, e\right\}\right)+\left(1-\right.$ full $\left._{v_{1}}(f)\right)$, and in Case $2 \gamma((S \cup\{e\})-\{a\})=$ $\gamma((S \cup\{e, f\})-\{a\})+$ full $_{v_{1}}(f)$ and $\gamma\left(\left\{f, f^{\prime}, e\right\}\right)=\gamma\left(\left\{f^{\prime}, e\right\}\right)-$ full $_{v_{1}}(f)$. Therefore:

$$
\begin{aligned}
\gamma((S \cup\{e\})-\{a\})+\gamma\left(\left\{f, f^{\prime}, e\right\}\right) & =\gamma((S \cup\{e, f\})-\{a\})+\gamma\left(\left\{f^{\prime}, e\right\}\right) \\
& \leq \lambda((S \cup\{e, f\})-\{a\})+\lambda\left(\left\{f^{\prime}, e\right\}\right) \\
& \leq \lambda((S \cup\{e\})-\{a\})+\lambda\left(\left\{f, f^{\prime}, e\right\}\right) \\
& \leq \lambda((S \cup\{e\})-\{a\})+\gamma\left(\left\{f, f^{\prime}, e\right\}\right),
\end{aligned}
$$

where (2) comes from (1) by the induction hypothesis and $C 1,(3)$ comes 
from (2) by submodularity, and (4) comes from (3) by the first half of the lemma. Therefore $\gamma((S \cup\{e\})-\{a\}) \leq \lambda((S \cup\{e\})-\{a\})$.

Suppose that $f$ is parallel to some element of $S-\{a\}$.
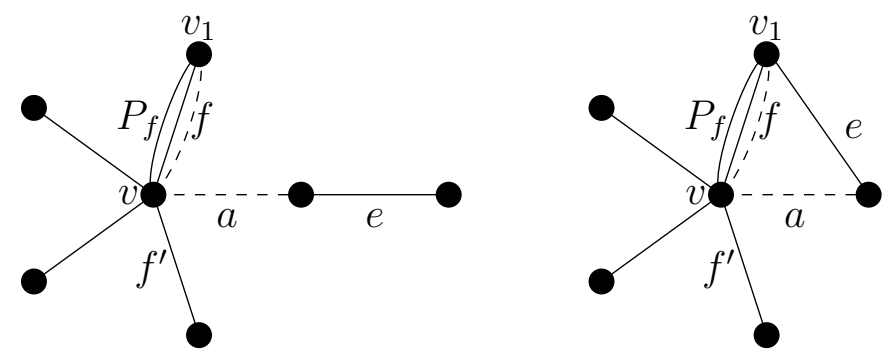

Then $\gamma((S \cup\{e\})-\{a\})=\gamma((S \cup\{e, f\})-\{a\})+$ full $_{v_{1}}\left(P_{f} \cup\{e\}\right)$ where $P_{f}$ is the collection of elements parallel to $f$ in $S$, and $\gamma\left(P_{f}\right)=\gamma\left(P_{f}-\{f\}\right)-$ full $_{v_{1}}\left(P_{f} \cup\{e\}\right)$. Therefore:

$$
\begin{aligned}
\gamma((S \cup\{e\})-\{a\})+\gamma\left(P_{f}\right) & =\gamma((S \cup\{e, f\})-\{a\})+\gamma\left(P_{f}-\{f\}\right) \\
& \leq \lambda((S \cup\{e, f\})-\{a\})+\lambda\left(P_{f}-\{f\}\right) \\
& \leq \lambda((S \cup\{e\})-\{a\})+\lambda\left(P_{f}\right) \\
& \leq \lambda((S \cup\{e\})-\{a\})+\gamma\left(P_{f}\right),
\end{aligned}
$$

where (2) comes from (1) by the induction hypothesis and Lemma 4.28, (3) comes from (2) by submodularity, and (4) comes from (3) by Lemma 4.28 . Therefore $\gamma((S \cup\{e\})-\{a\}) \leq \lambda((S \cup\{e\})-\{a\})$.

Finally suppose that $f$ is parallel to $a$.

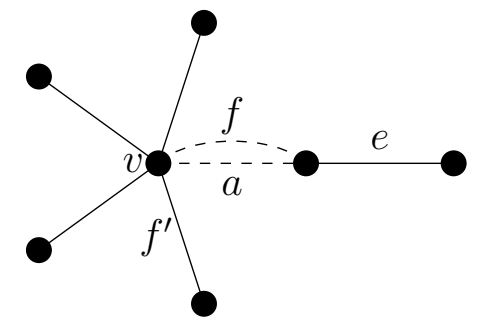

Then $\gamma((S \cup\{e\})-\{a\})=\gamma((S \cup\{e, f\})-\{a\})$ and $\gamma\left(\left\{f, f^{\prime}, e\right\}\right)=\gamma\left(\left\{f^{\prime}, e\right\}\right)$. 
Therefore:

$$
\begin{aligned}
\gamma((S \cup\{e\})-\{a\})+\gamma\left(\left\{f, f^{\prime}, e\right\}\right) & =\gamma((S \cup\{e, f\})-\{a\})+\gamma\left(\left\{f^{\prime}, e\right\}\right) \\
& \leq \lambda((S \cup\{e, f\})-\{a\})+\lambda\left(\left\{f^{\prime}, e\right\}\right) \\
& \leq \lambda((S \cup\{e\})-\{a\})+\lambda\left(\left\{f, f^{\prime}, e\right\}\right) \\
& \leq \lambda((S \cup\{e\})-\{a\})+\gamma\left(\left\{f, f^{\prime}, e\right\}\right),
\end{aligned}
$$

where (2) comes from (1) by the induction hypothesis and $C 1,(3)$ comes from (2) by submodularity, and (4) comes from (3) by the first half of the lemma.

The result follows.

This next lemma considers at structures that look something like the one shown below:

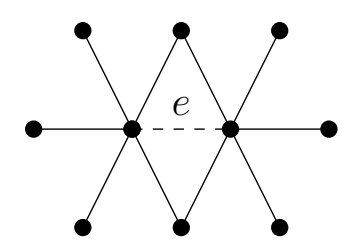

Lemma 4.32. Let e be an edge of $G$ between vertices $v_{1}$ and $v_{2}$. Let $S_{1}$ be a star at $v_{1}$ such that $e \in S_{1}$, and $S_{2}$ be a star at $v_{2}$ such that $e \in S_{2}$. Then $\lambda\left(\left(S_{1} \cup S_{2}\right)-\{e\}\right)=\gamma\left(\left(S_{1} \cup S_{2}\right)-\{e\}\right)$.

Proof. We first show that $\lambda\left(\left(S_{1} \cup S_{2}\right)-\{e\}\right) \geq \gamma\left(\left(S_{1} \cup S_{2}\right)-\{e\}\right)$. The result holds for $\left|\left(S_{1} \cup S_{2}\right)-\{e\}\right| \leq 2$ by $C 1$ on $\lambda$. Suppose for the purposes of induction that the result holds for all $\left(S_{1} \cup S_{2}\right)-\{e\}$ such that $\mid\left(S_{1} \cup S_{2}\right)-$ $\{e\} \mid<m$.

Suppose that $\left|\left(S_{1} \cup S_{2}\right)-\{e\}\right|=m \geq 3$. If at least one of $S_{1}$ and $S_{2}$ has cardinality less than three, then the result holds by Lemma 4.31 so we can assume that $\left|S_{1}\right| \geq 3$ and $\left|S_{2}\right| \geq 3$. Let $f$ be an edge in $S_{1} \cup S_{2}$ that is not $e$ and is not parallel to $e$. If no such $f$ exists then all edges in $S_{1} \cup S_{2}$ are parallel to $e$ and therefore $S_{1} \cup S_{2}-\{e\}$ is a parallel set and the result holds by Lemma 4.28. Therefore we assume such an $f$ exists and without loss of generality we shall assume that it is in $S_{1}$.

Let $V(\{f\})=\left\{v_{1}, v_{3}\right\}$ (note that $v_{2} \neq v_{3}$ as $f$ is not parallel to $e$ ). Let $S$ be the complete star of $G$ at $v_{3}$ and let the collection of all edges of $S$ that are 
also in $S_{1}$ be $P_{1}$ and the collection of all edges that are also in $S_{2}$ be $P_{2}$. Let $P=P_{1} \cup P_{2}$.

The pictures below aim to illustrate this situation, although do not, by any means, cover all possible configurations. In the pictures below, the red edges are the edges of $S-\left(S_{1} \cup S_{2}\right)$ and in the second picture $S_{1}-\{e\}$ is shown in blue.

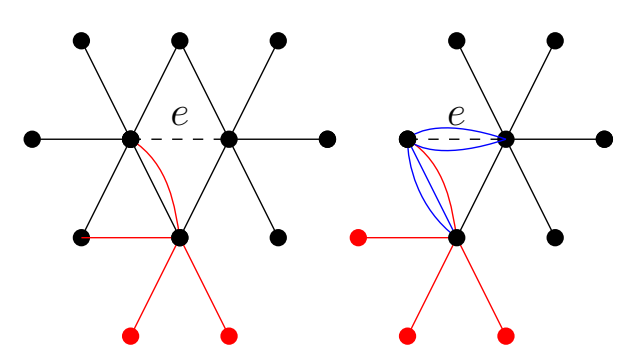

Suppose that $S_{1}-\{e\} \neq P_{1}$ and $S_{2}-\{e\} \neq P_{2}$. This means that there is some edge in $S_{1}-\{e\}$ that is not in $S$ and some edge in $S_{2}-\{e\}$ that is not in $S$. Then $\gamma\left(\left(S_{1} \cup S_{2}\right)-\{e\}\right)=\gamma\left(\left(\left(S_{1} \cup S_{2}\right)-\{e\}\right)-P\right)+\left(1-\right.$ full $\left.v_{3}(P)\right)$, and $\gamma(S \cup\{e\})=\gamma(S \cup\{e\}-P)-\left(1-\right.$ full $\left.v_{3}(P)\right)$ (note that this holds when $S=P)$. Therefore:

$$
\begin{aligned}
& \gamma\left(\left(S_{1} \cup S_{2}\right)-\{e\}\right)+\gamma(S \cup\{e\}) \\
& \quad=\gamma\left(\left(\left(S_{1} \cup S_{2}\right)-\{e\}\right)-P\right)+\gamma((S \cup\{e\})-P) \\
& \quad \leq \lambda\left(\left(\left(S_{1} \cup S_{2}\right)-\{e\}\right)-P\right)+\lambda((S \cup\{e\})-P) \\
& \quad=\lambda\left(E-\left(\left(\left(S_{1} \cup S_{2}\right)-\{e\}\right)-P\right)\right)+\lambda((S \cup\{e\})-P) \\
& \quad \leq \lambda\left(E-\left(\left(S_{1} \cup S_{2}\right)-\{e\}\right)\right)+\lambda(S \cup\{e\}) \\
& \quad \leq \lambda\left(\left(S_{1} \cup S_{2}\right)-\{e\}\right)+\gamma(S \cup\{e\}),
\end{aligned}
$$

where (2) follows from (1) by the equalities given above, (3) follows from (2) by the induction hypothesis and Lemma 4.31, (4) follows from (3) by symmetry, (5) follows from (4) by submodularity and (6) follows from (5) by symmetry and Lemma 4.30. Therefore $\gamma\left(\left(S_{1} \cup S_{2}\right)-\{e\}\right) \leq \lambda\left(\left(S_{1} \cup S_{2}\right)-\{e\}\right)$. Suppose that either $S_{1}-\{e\}=P_{1}$ or $S_{2}-\{e\}=P_{2}$. If $S_{1}-\{e\}=P_{1}$ and $S_{2}-\{e\}=P_{2}$ then, as $P_{1} \cup P_{2}$ share vertex $v_{3}$, the collection of edges $\left(S_{1} \cup S_{2}\right)-\{e\}$ is a star, and so the result holds by Lemma 4.28. Therefore we assume that only one of $S_{1}-\{e\}=P_{1}$ and $S_{2}-\{e\}=P_{2}$ hold. Without loss of generality let $S_{1}-\{e\}=P_{1}$ and recall that $f \in S_{1}-\{e\}=P_{1}$. We 
shall split into two cases here, one for when $S=P$ and one for when $S \neq P$. First consider the case where $S=P$.

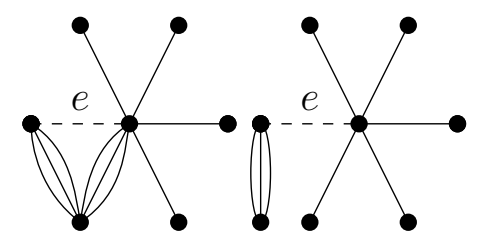

Then $\gamma\left(\left(S_{1} \cup S_{2}\right)-\{e\}\right)=\gamma\left(\left(S_{1} \cup S_{2}\right)-\{e, f\}\right)-1$ and $\gamma(\{e, f\})=\gamma(\{e\})+1$. Therefore:

$$
\begin{aligned}
\gamma\left(\left(S_{1} \cup S_{2}\right)-\{e\}\right)+\gamma(\{e, f\}) & =\gamma\left(\left(S_{1} \cup S_{2}\right)-\{e, f\}\right)+\gamma(\{e\}) \\
& =\gamma\left(E-\left(\left(S_{1} \cup S_{2}\right)-\{e, f\}\right)\right)+\gamma(\{e\}) \\
& \leq \lambda\left(E-\left(\left(S_{1} \cup S_{2}\right)-\{e, f\}\right)\right)+\lambda(\{e\}) \\
& \leq \lambda\left(E-\left(\left(S_{1} \cup S_{2}\right)-\{e\}\right)\right)+\lambda(\{e, f\}) \\
& =\lambda\left(\left(S_{1} \cup S_{2}\right)-\{e\}\right)+\gamma(\{e, f\}),
\end{aligned}
$$

where (2) follows from (1) by symmetry, (3) follows from (2) by the induction hypothesis and C1, (4) follows from (3) by submodularity and (5) follows from (4) by symmetry and $C 1$. Therefore $\gamma\left(\left(S_{1} \cup S_{2}\right)-\{e\}\right) \leq \lambda\left(\left(S_{1} \cup S_{2}\right)-\{e\}\right)$. Now consider the case where $S \neq P$ and again note that $f \in P_{1}$. As $S \neq P$, let $g$ be an element of $S-P$. Note that there are at least two edges in $P_{1}$ as $P_{1}=S_{1}-\{e\}$ and $\left|S_{1}\right| \geq 3$. On the diagram below edges of $S-P$ are shown in red.

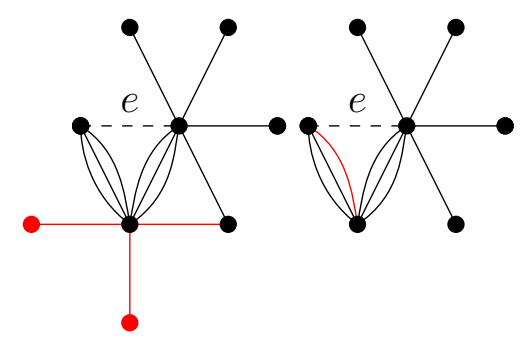

Then $\gamma\left(\left(S_{1} \cup S_{2}\right)-\{e\}\right)=\gamma\left(\left(S_{1} \cup S_{2}\right)-\{e, f\}\right)$ and $\gamma(\{e, f, g\})=\gamma(\{e, g\})$ 
as $\left|S_{1}\right| \geq 3$. Therefore:

$$
\begin{aligned}
& \gamma\left(\left(S_{1} \cup S_{2}\right)-\{e\}\right)+\gamma(\{e, f, g\}) \\
& \quad=\gamma\left(\left(S_{1} \cup S_{2}\right)-\{e, f\}\right)+\gamma(\{e, g\}) \\
& \quad \leq \lambda\left(\left(S_{1} \cup S_{2}\right)-\{e, f\}\right)+\lambda(\{e, g\}) \\
& \quad=\lambda\left(E-\left(\left(S_{1} \cup S_{2}\right)-\{e, f\}\right)\right)+\lambda(\{e, g\}) \\
& \quad \leq \lambda\left(E-\left(\left(S_{1} \cup S_{2}\right)-\{e\}\right)\right)+\lambda(\{e, f, g\}) \\
& \quad \leq \lambda\left(\left(S_{1} \cup S_{2}\right)-\{e\}\right)+\gamma(\{e, f, g\}),
\end{aligned}
$$

where (2) follows from (1) by the equalities given above, (3) follows from (2) by the induction hypothesis and $C 1$, (4) follows from (3) by symmetry, (5) follows from (4) by submodularity and (6) follows from (5) by symmetry and Lemma 4.30 .

Therefore $\gamma\left(\left(S_{1} \cup S_{2}\right)-\{e\}\right) \leq \lambda\left(\left(S_{1} \cup S_{2}\right)-\{e\}\right)$

Next we show that $\lambda\left(\left(S_{1} \cup S_{2}\right)-\{e\}\right) \leq \gamma\left(\left(S_{1} \cup S_{2}\right)-\{e\}\right)$. Again the result holds for $\left(S_{1} \cup S_{2}\right)-\{e\}$ such that $\left|\left(S_{1} \cup S_{2}\right)-\{e\}\right| \leq 2$. Suppose that $\left(S_{1} \cup S_{2}\right)$ contains no triangles.

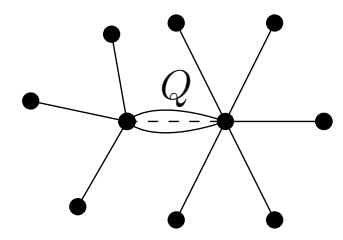

Let $Q$ be the collection of edges of $S_{1} \cup S_{2}$ that are parallel to $e$ and notice that $\gamma\left(S_{1}-\{e\}\right)+\gamma\left(S_{2}-\{e\}\right)=\gamma\left(\left(S_{1} \cup S_{2}\right)-\{e\}\right)+\gamma(Q-\{e\})$ (note that this works even if $Q=\{e\}$ and if $S_{1}, S_{2}$ or both are equal to $Q$ ). Therefore:

$$
\begin{aligned}
\gamma\left(\left(S_{1} \cup S_{2}\right)-\{e\}\right)+\gamma(Q-\{e\}) & =\gamma\left(S_{1}-\{e\}\right)+\gamma\left(S_{2}-\{e\}\right) \\
& =\lambda\left(S_{1}-\{e\}\right)+\lambda\left(S_{2}-\{e\}\right) \\
& \geq \lambda\left(\left(S_{1} \cup S_{2}\right)-\{e\}\right)+\lambda(Q-\{e\}) \\
& =\lambda\left(\left(S_{1} \cup S_{2}\right)-\{e\}\right)+\gamma(Q-\{e\}),
\end{aligned}
$$

where (2) follows from (1) by Lemma 4.28, (3) follows from (2) by submodularity and (4) follows from (3) by Lemma 4.28 .

Suppose that $S_{1} \cup S_{2}$ is not triangle free. We will proceed by induction on 
the number of parallel triangles in $S_{1} \cup S_{2}$. The following claim provides the base case.

Claim 4.32.1. If $S_{1} \cup S_{2}$ contains exactly one parallel triangle then $\lambda\left(\left(S_{1} \cup S_{2}\right)-\{e\}\right) \leq \gamma\left(\left(S_{1} \cup S_{2}\right)-\{e\}\right)$.

Proof. Let the maximal parallel subsets of the parallel triangle be $P_{1}, P_{2}, Q$ where $P_{1} \subseteq S_{1}$ and $P_{2} \subseteq S_{2}$.

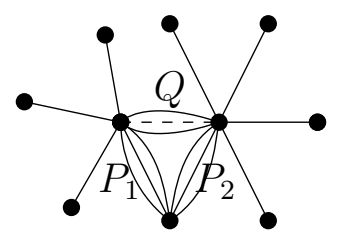

Then $\gamma\left(\left(S_{1} \cup P_{2}\right)-\{e\}\right)+\gamma\left(\left(S_{2} \cup P_{1}\right)-\{e\}\right)=\gamma\left(\left(S_{1} \cup S_{2}\right)-\{e\}\right)+$ $\gamma\left(P_{1} \cup P_{2}\right)$. Therefore:

$$
\begin{aligned}
& \gamma\left(\left(S_{1} \cup S_{2}\right)-\{e\}\right)+\gamma\left(P_{1} \cup P_{2}\right) \\
& \quad=\gamma\left(\left(S_{1} \cup P_{2}\right)-\{e\}\right)+\gamma\left(\left(S_{2} \cup P_{1}\right)-\{e\}\right) \\
& \quad \geq \lambda\left(\left(S_{1} \cup P_{2}\right)-\{e\}\right)+\lambda\left(\left(S_{2} \cup P_{1}\right)-\{e\}\right) \\
& \quad \geq \lambda\left(\left(S_{1} \cup S_{2}\right)-\{e\}\right)+\lambda\left(P_{1} \cup P_{2}\right) \\
& \quad=\lambda\left(\left(S_{1} \cup S_{2}\right)-\{e\}\right)+\gamma\left(P_{1} \cup P_{2}\right),
\end{aligned}
$$

where (2) follows from (1) by the equalities given above, (3) follows from (2) by Lemma 4.30, (4) follows from (3) by submodularity, (5) follows from (4) by Lemma 4.28. Therefore the claim holds.

Now suppose that $S_{1} \cup S_{2}$ contains at least two parallel triangles, two of which we shall call $P_{1}, P_{2}, Q$ and $P_{1}^{\prime}, P_{2}^{\prime}, Q$ where $P_{1}, P_{1}^{\prime} \in S_{1}$ and $P_{2}, P_{2}^{\prime} \in S_{2}$.

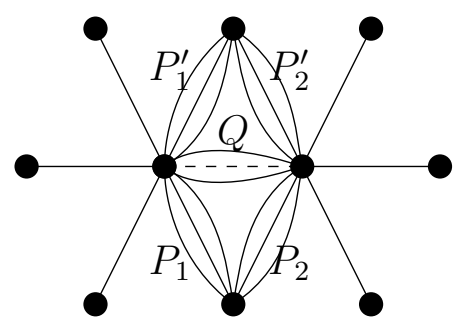


Note that $\gamma\left(\left(S_{1} \cup S_{2}\right)-\left(P_{1} \cup\{e\}\right)\right)+\gamma\left(\left(S_{1} \cup S_{2}\right)-\left(P_{2}^{\prime} \cup\{e\}\right)=\gamma\left(\left(S_{1} \cup S_{2}\right)-\right.\right.$ $\{e\})+\gamma\left(\left(S_{1} \cup S_{2}\right)-\left(P_{1} \cup P_{2}^{\prime} \cup\{e\}\right)\right)$. Therefore:

$$
\begin{aligned}
\gamma\left(\left(S_{1} \cup S_{2}\right)\right. & -\{e\})+\gamma\left(\left(S_{1} \cup S_{2}\right)-\left(\{e\} \cup P_{1} \cup P_{2}^{\prime}\right)\right) \\
& =\gamma\left(\left(S_{1} \cup S_{2}\right)-\left(\{e\} \cup P_{1}\right)\right)+\gamma\left(\left(S_{1} \cup S_{2}\right)-\left(\{e\} \cup P_{2}^{\prime}\right)\right) \\
& \geq \lambda\left(\left(S_{1} \cup S_{2}\right)-\left(\{e\} \cup P_{1}\right)\right)+\lambda\left(\left(S_{1} \cup S_{2}\right)-\left(\{e\} \cup P_{2}^{\prime}\right)\right) \\
& \geq \lambda\left(\left(S_{1} \cup S_{2}\right)-\{e\}\right)+\lambda\left(\left(S_{1} \cup S_{2}\right)-\left(\{e\} \cup P_{1} \cup P_{2}^{\prime}\right)\right) \\
& \geq \lambda\left(\left(S_{1} \cup S_{2}\right)-\{e\}\right)+\gamma\left(\left(S_{1} \cup S_{2}\right)-\left(\{e\} \cup\left(P_{1} \cup P_{2}^{\prime}\right)\right)\right.
\end{aligned}
$$

where (2) follows from (1) by equalities given above, (3) follows from (2) by the induction hypothesis, (4) follows from (3) by submodularity and (5) follows from (4) by the first part of the lemma. Therefore $\lambda\left(\left(S_{1} \cup S_{2}\right)-\{e\}\right) \leq$ $\gamma\left(\left(S_{1} \cup S_{2}\right)-\{e\}\right)$.

Lemma 4.33. Let e be an edge of $G$ between vertices $v_{1}$ and $v_{2}$ and let $S_{1}$ be a star at $v_{1}$ such that $e \in S_{1}$, and $S_{2}$ be a star at $v_{2}$ such that $e \in S_{2}$. Then $\lambda\left(S_{1} \cup S_{2}\right) \leq \gamma\left(S_{1} \cup S_{2}\right)$.

Proof. Let $Q$ be the collection of edges of $S_{1} \cup S_{2}$ that are parallel to $e$. If $S_{1} \cup S_{2}=Q$ or one of $S_{1}, S_{2}$ is contained in $Q$ then the result holds by Lemma 4.28. Our proof will be by induction on the number of triangles in $S_{1} \cup S_{2}$ and the following claim will provide our base case:

Claim 4.33.1. When $S_{1} \cup S_{2}$ is triangle free $\lambda\left(S_{1} \cup S_{2}\right) \leq \gamma\left(S_{1} \cup S_{2}\right)$.

Proof. Suppose that $S_{1} \cup S_{2}$ is a triangle free subgraph of $G$. If the only edges in $S_{1}$ or $S_{2}$ are parallel to $e$, then the result holds by Lemma 4.28 , so we will assume that both $S_{1}$ and $S_{2}$ contain at least one edge each that is not parallel to $e$.

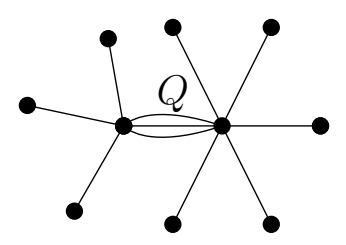

Observe that $\gamma\left(S_{1} \cup Q\right)+\gamma\left(S_{2} \cup Q\right)=\gamma\left(S_{1} \cup S_{2}\right)+2$ and note that by Lemma $4.28 \gamma\left(S_{1} \cup Q\right)=\lambda\left(S_{1} \cup Q\right), \gamma\left(S_{2} \cup Q\right)=\lambda\left(S_{2} \cup Q\right)$, and 
$\gamma(Q)=2=\lambda(Q)$. Therefore:

$$
\begin{aligned}
\gamma\left(S_{1} \cup S_{1}\right)+\gamma(Q) & =\gamma\left(S_{1} \cup Q\right)+\gamma\left(S_{2} \cup Q\right) \\
& =\lambda\left(S_{1} \cup Q\right)+\lambda\left(S_{2} \cup Q\right) \\
& \geq \lambda\left(S_{1} \cup S_{2}\right)+\lambda(Q) \\
& =\lambda\left(S_{1} \cup S_{2}\right)+\gamma(Q),
\end{aligned}
$$

where the first equality holds by the equalities given above, (2) follows from (1) by Lemma 4.28, (3) follows from (2) by submodularity and (4) follows from (3) by Lemma 4.28. Therefore $\lambda\left(S_{1} \cup S_{2}\right) \leq \gamma\left(S_{1} \cup S_{2}\right)$.

Suppose that the result holds for subgraphs with less than $k$ parallel triangles. Now suppose that $S_{1} \cup S_{2}$ contains $k \geq 1$ parallel triangles. Let one of the triangles of $S_{1} \cup S_{2}$ be $P_{1}, P_{2}, Q$ (note that $Q$ will be in all parallel triangles), where $P_{1} \in S_{1}$ and $P_{2} \in S_{2}$.

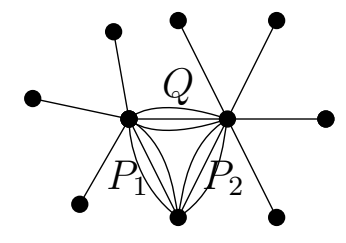

Suppose one of $S_{1}$ and $S_{2}$ contains no edges other than those in $Q$ or in $P_{1} \cup P_{2}$. Then the result holds by Lemma 4.30.

So suppose that both $S_{1}$ and $S_{2}$ contain an element that is not parallel to any element of $Q$ and is not contained in $P_{1} \cup P_{2}$. Then $v_{1}$ is not full with respect to $P_{1} \cup Q$ and $v_{2}$ is not full with respect to $P_{2} \cup Q$. Let $V\left(P_{1}\right)=\left\{v_{1}, v_{3}\right\}$ and note that $\gamma\left(S_{1} \cup P_{2} \cup Q\right)=\gamma\left(\left(S_{1}-P_{1}\right) \cup P_{2} \cup Q\right)-$ full $_{v_{3}}\left(P_{1} \cup P_{2}\right)-$ full $_{v_{1}}\left(S_{1} \cup Q\right)$ 
and $\gamma\left(\left(S_{1} \cup S_{2}\right)-P_{1}\right)=\gamma\left(S_{1} \cup S_{2}\right)+$ full $_{v_{3}}\left(P_{1} \cup P_{2}\right)+$ full $_{v_{1}}\left(S_{1} \cup Q\right)$. Therefore:

$$
\begin{aligned}
\gamma\left(S_{1} \cup S_{2}\right)+\gamma\left(\left(S_{1}-P_{1}\right) \cup P_{2} \cup Q\right) & =\gamma\left(\left(S_{1} \cup S_{2}\right)-P_{1}\right)+\gamma\left(S_{1} \cup P_{2} \cup Q\right) \\
& \geq \lambda\left(\left(S_{1} \cup S_{2}\right)-P_{1}\right)+\lambda\left(S_{1} \cup P_{2} \cup Q\right) \\
& \geq \lambda\left(S_{1} \cup S_{2}\right)+\lambda\left(\left(S_{1}-P_{1}\right) \cup P_{2}\right) \\
& =\lambda\left(S_{1} \cup S_{2}\right)+\gamma\left(\left(S_{1}-P_{1}\right) \cup P_{2} \cup Q\right),
\end{aligned}
$$

where the first equality follows from the equalities given above, (2) follows from (1) by the induction hypothesis and Lemma 4.30, (3) follows from (2) by submodularity and (4) follows from (3) by Lemma 4.32 using stars $\left(S_{1}-\right.$ $\left.P_{1}\right) \cup\{e\}$ and $P_{2} \cup\{e\}$ for some $e \in P_{1}$.

Therefore $\gamma\left(S_{1} \cup S_{2}\right) \geq \lambda\left(S_{1} \cup S_{2}\right)$.

Lemma 4.34. For all $Y \subseteq E(G)$, we have $\lambda(Y) \leq \gamma(Y)$.

Proof. Let $Y$ be a minimal subset of $E(G)$ for which the lemma fails. In other words, let $Y$ be such that $\lambda(Y)>\gamma(Y)$ and every $Y^{\prime} \subseteq Y$ has $\lambda\left(Y^{\prime}\right) \leq \gamma\left(Y^{\prime}\right)$. If $\lambda(Y)>\gamma(Y)$ then, by $C 1,|Y|>2$.

Suppose that $e$ is an isolated edge in $Y$, that is, $e$ is an edge of $Y$ that is adjacent to no other edge of $Y$. The diagram below shows that subgraph of $G$ whose edges are the edges in $Y$.



Let $V(\{e\})=\left\{v_{1}, v_{2}\right\}$, and let $f \in Y-\{e\}$. Then $\gamma(Y-\{e\})=\gamma(Y)-(1-$ full $\left._{v_{1}}(e)\right)-\left(1-\right.$ full $\left._{v_{2}}(e)\right)$, and $\gamma\left(\{e, f\}=\gamma(\{f\})+\left(1-\right.\right.$ full $\left._{v_{1}}(e)\right)+\left(1-\right.$ full $\left._{v_{2}}(e)\right)$. 
Therefore:

$$
\begin{aligned}
\gamma(Y)+\gamma(\{f\}) & =\gamma(Y-\{e\})+\gamma(\{e, f\}) \\
& \geq \lambda(Y-\{e\})+\lambda(\{e, f\}) \\
& \geq \lambda(Y)+\lambda(\{f\}) \\
& =\lambda(Y)+\gamma(\{f\}),
\end{aligned}
$$

where the first equality holds by the equalities given above, (2) follows from (1) by minimality of $Y$ and $C 1,(3)$ follows from (2) by submodularity and (4) follows from (3) by $C 1$.

Therefore $\gamma(Y) \geq \lambda(Y)$ contradicting the assumption that $Y$ is a minimal subset of $E(G)$ for which $\lambda(Y)>\gamma(Y)$. Therefore $Y$ cannot contain an isolated edge.

Suppose that $e$ is an element of $Y$ which is incident with exactly one vertex in $V(Y-\{e\})$. Again, the diagram below shows that subgraph of $G$ whose edges are the edges in $Y$.



Let $V(\{e\})=\left\{v_{1}, v_{2}\right\}$ where $v_{1} \in V(Y-\{e\})$. Let $S$ be a maximal subset of $Y$ that is a star of $G$ at $v_{1}$ (note that $S$ contains at least two elements). Then $\gamma(Y-\{e\})=\gamma(Y)-\left(1-\operatorname{full}_{v_{2}}(e)\right)+\operatorname{full}_{v_{1}}(Y)$, and $\gamma(S)=\gamma(S-$ $\{e\})+\left(1-\operatorname{full}_{v_{2}}(e)\right)-\operatorname{full}_{v_{1}}(S)$. As full $v_{1}(S)=\operatorname{full}_{v_{1}}(Y)$ :

$$
\begin{aligned}
\gamma(Y)+\gamma(S-\{e\}) & =\gamma(Y-\{e\})+\gamma(S) \\
& \geq \lambda(Y-\{e\})+\lambda(S) \\
& \geq \lambda(Y)+\lambda(S-\{e\}) \\
& =\lambda(Y)+\gamma(S-\{e\})
\end{aligned}
$$

where the first equality holds by the equalities given above, (2) follows from (1) by the minimality of $Y$ and Lemma 4.28, (3) follows from (2) by submodularity and (4) follows from (2) by Lemma 4.28. Therefore $\gamma(Y) \geq \lambda(Y)$ contradicting the assumption that $Y$ is a minimal subset of $E(G)$ for which 
$\lambda(Y)>\gamma(Y)$. Therefore every edge of $Y$ must be incident with two vertices in $V(Y-\{e\})$.

Suppose that $e$ is an element of $Y$ which is incident with two vertices in $V(Y-\{e\})$.



Let $V(\{e\})=\left\{v_{1}, v_{2}\right\}$. Let $S_{1}$ be a maximal subset of $Y$ that is a star of $G$ at $v_{1}$ and let $S_{2}$ be a maximal subset of $Y$ that is a star of $G$ at $v_{2}$ (note that $S_{1}$ and $S_{2}$ both contain at least two elements). Then $\gamma(Y-\{e\})=\gamma(Y)+$ full $_{v_{1}}(Y)+\operatorname{full}_{v_{2}}(Y)$, and $\gamma\left(S_{1} \cup S_{2}\right)=\gamma\left(S_{1} \cup S_{2}-\{e\}\right)-\operatorname{full}_{v_{1}}\left(S_{1}\right)-\operatorname{full}_{v_{2}}\left(S_{2}\right)$. As full $v_{1}\left(S_{1}\right)=\operatorname{full}_{v_{1}}(Y)$ and full $v_{v_{2}}\left(S_{2}\right)=\operatorname{full}_{v_{2}}(Y)$ :

$$
\begin{aligned}
\gamma(Y)+\gamma\left(S_{1} \cup S_{2}-\{e\}\right) & =\gamma(Y-\{e\})+\gamma\left(S_{1} \cup S_{2}\right) \\
& \geq \lambda(Y-\{e\})+\lambda\left(S_{1} \cup S_{2}\right) \\
& \geq \lambda(Y)+\lambda\left(S_{1} \cup S_{2}-\{e\}\right) \\
& =\lambda(Y)+\gamma\left(S_{1} \cup S_{2}-\{e\}\right),
\end{aligned}
$$

where the first equality holds by the equalities given above, (2) follows from (1) by minimality of $Y$ and Lemma 4.33. (3) follows from (2) by submodularity and (4) follows from (3) by Lemma 4.32. Therefore $\gamma(Y) \geq \lambda(Y)$ contradicting the assumption that $Y$ is a minimal subset of $E(G)$ for which $\lambda(Y)>\gamma(Y)$.

As we cannot find a minimal $Y$ for which $\lambda(Y)>\gamma(Y)$, for all $Y \subseteq E(G)$ we have $\gamma(Y) \geq \lambda(Y)$.

Lemma 4.35. For all $Y \subseteq E(G), \lambda(Y) \geq \gamma(Y)$.

Proof. We will consider a set $Y$ which is maximal with respect to $\lambda(Y)<$ $\gamma(Y)$, in other words for any $A \subseteq E(G)-Y, \gamma(Y \cup A) \leq \lambda(Y \cup A)$. Note that, by $C 1$ and symmetry, $\lambda(Y)=\gamma(Y)$ for all $Y \subseteq E(G)$ such that $|Y| \geq$ $|E(G)|-2$. 
Suppose that $V(G) \neq V(Y)$. Then, either there is an edge $e$ with $V(e)=$ $\left\{v_{1}, v_{2}\right\}$ where $v_{1} \in V(Y)$ or $Y$ is a separator of $G$.

Suppose first that $Y$ is a separator of $G$. Note that $\gamma(Y)+\gamma(\{e\})=\gamma(Y \cup\{e\})$ if $e$ is in $E-E(Y)$. Therefore:

$$
\begin{aligned}
\gamma(Y)+\gamma(\{e\}) & =\gamma(Y \cup\{e\}) \\
& \leq \lambda(Y \cup\{e\}) \\
& \leq \lambda(Y)+\lambda(\{e\}) \\
& =\lambda(Y)+\gamma(\{e\}),
\end{aligned}
$$

(2) follows from (1) by maximality of $Y$, (3) follows from (2) by submodularity and (4) follows from (3) by C1. Therefore in this case $\gamma(Y) \leq \lambda(Y)$.

Next suppose that $Y$ is not a separator of $G$. Therefore there is some edge $e$ such that $V(\{e\})=\left\{v_{1}, v_{2}\right\}$ where $v_{1} \in V(Y)$ and $v_{2} \notin V(Y)$.

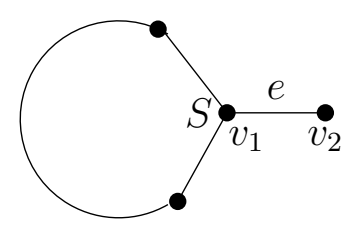

Let the maximal subset of $Y \cup\{e\}$ that is a star of $G$ at $v_{1}$ be $S$. Note that $\gamma(Y \cup\{e\})=\gamma(Y)+\left(1-\right.$ full $\left._{v_{2}}(e)\right)-$ full $_{v_{1}}(S)$ and $\gamma(S-\{e\})=\gamma(S)-(1-$ full $\left._{v_{2}}(e)\right)+$ full $_{v_{1}}(S)$. Therefore:

$$
\begin{aligned}
\gamma(Y)+\gamma(S) & =\gamma(Y \cup\{e\})+\gamma(S-\{e\}) \\
& \leq \lambda(Y \cup\{e\})+\lambda(S-\{e\}) \\
& \leq \lambda(Y)+\lambda(S) \\
& <\lambda(Y)+\gamma(S),
\end{aligned}
$$

where the first equality follows from the equalities given above, (2) follows from (1) by maximality of $Y$ and Lemma 4.28, (3) follows from (2) by submodularity and (4) follows from (3) by assumption and Lemma 4.28; a contradiction.

Therefore every vertex of $G$ must be contained in $V(Y)$.

As the result holds for $E(G)$ and we are considering $Y$ to be a first subset of 
$E(G)$ for which the result fails, there must be an edge in $E(G)$ that is not in $Y$. Let $e$ be such an edge and suppose $e$ is incident with vertices $v_{1}$ and $v_{2}$ (both of which are in $V(Y)$ ).

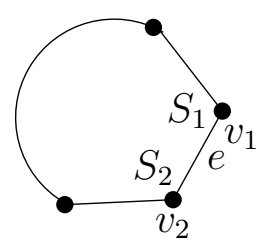

Consider the maximal subset of $Y \cup\{e\}$ that is a star at $v_{1}$ which we shall call $S_{1}$ and the maximal subset of $Y \cup\{e\}$ that is a star at $v_{2}$ which we shall call $S_{2}$. Then $\gamma\left(S_{1} \cup S_{2}\right)=\gamma\left(\left(S_{1} \cup S_{2}\right)-\{e\}\right)-\operatorname{full}_{v_{1}}\left(S_{1}\right)-$ full $_{v_{2}}\left(S_{2}\right)$ and $\gamma(Y)=$ $\gamma(Y \cup\{e\})+\operatorname{full}_{v_{1}}(Y \cup\{e\})+\operatorname{full}_{v_{2}}(Y \cup\{e\})$. As full $_{v_{1}}\left(S_{1}\right)=\operatorname{full}_{v_{1}}(Y \cup\{e\})$ and $\operatorname{full}_{v_{2}}\left(S_{2}\right)=\operatorname{full}_{v_{2}}(Y \cup\{e\})$

$$
\begin{aligned}
\gamma(Y)+\gamma\left(S_{1} \cup S_{2}\right) & =\gamma(Y \cup\{e\})+\gamma\left(S_{1} \cup S_{2}-\{e\}\right) \\
& \leq \lambda(Y \cup\{e\})+\lambda\left(S_{1} \cup S_{2}-\{e\}\right) \\
& \leq \lambda(Y)+\lambda\left(S_{1} \cup S_{2}\right) \\
& <\lambda(Y)+\gamma\left(S_{1} \cup S_{2}\right),
\end{aligned}
$$

where the first equality follows from the equalities given above, (2) follows from (1) by maximality of $Y$ and Lemma 4.32, (3) follows from (2) by submodularity and (4) follows from (3) by assumption and Lemma 4.34. A contradiction.

Therefore there does not exist a set $Y$ for which the theorem fails so $\lambda(Y) \geq$ $\gamma(Y)$

Combining our last two lemmas we immediately get a proof of the following theorem:

Theorem 4.25, Let $G$ be a graph with connectivity function $\gamma$. Let $\lambda$ be a connectivity function on $E(G)$ such that $\lambda(X)=\gamma(X)$ whenever $X \subseteq E$ satisfies any one of the following:

$C 1|X| \leq 2$

C2 $X$ is a complete star of $G$, 
C3 $X$ is an almost-complete star of $G$,

$C_{4} X$ is a broken star of $G$,

C5 $X$ is a triangle, maximal parallel triangle, or $X \cup\{e\}$ is a maximal parallel triangle for some $e \in E(G)-E(X)$,

C6 $X$ is a parallel class in $G$.

Then $\lambda(X)=\gamma(X)$, for all $X \subseteq E$.

Theorem 4.1. There is some polynomial $p$ such that, given an arbitrary connectivity function $\lambda$, we are able to establish whether or not $\lambda$ is graphic in at most $p(n)$ evaluations of the connectivity function, where $n$ is the size of the set on which $\lambda$ is based.

Earlier in this section we saw an example of a graph that could be built from a connectivity function, $\lambda$, according to our algorithm, but did not have connectivity function $\lambda$. This means that there is some case where not all $C 1-C 6$ are redundant. However, we do not show that $C 1-C 6$ is a minimal set of conditions and the example used to show that checking is sometimes necessary is a graph whose connectivity function does not generally behave nicely. This leaves us with the following open questions:

Open Question 1 Are C1-C6 a minimal set of conditions for checking $\gamma=\lambda$ ?

Open Question 2 Are any such conditions necessary if we can build a graph that would have $\lambda$ as its connectivity function were $\lambda$ graphic, and this graph does not lie within some particular small collection of graphs (such as the collection of graphs that cannot be built identically, when we do not consider loops and isolated vertices, from the connectivity function). 


\section{Chapter 5}

\section{Matroidal Connectivity Functions}

Definition 5.1. A connectivity function, $\lambda$, is matroidal, if there is some matroid $M$ such that, $\mu_{M}=\lambda$ where $\mu_{M}$ is the connectivity function of $M$.

This section focuses on recognising when a connectivity function is matroidal. Certainly not every connectivity function is matroidal, as all connectivity functions that come from a matroid are unitary. In [8] an example is given of a unitary connectivity function that is not matroidal. This example is described below:

$$
\begin{aligned}
& \lambda:\{a, b, c, d\} \rightarrow Z_{\geq 0} \\
& \lambda(\{i\})=1 \text { for } i \in\{a, b, c, d\} \\
& \lambda(\{a, b\})=1 \\
& \lambda(\{a, c\})=1 \\
& \lambda(\{a, d\})=2
\end{aligned}
$$

The remaining values can be found by symmetry. This connectivity function can be obtained by taking the connectivity function of a graph that is a cycle of length four, and dividing this connectivity function by two.

In Section 5.1 we give an alternate, albeit similar, proof of a theorem of Lemos. Specifically the theorem is that if two matroids, $M_{1}$ and $M_{2}$ have 
the same connectivity function and $r\left(M_{1}\right) \neq r\left(M_{2}\right)$, then $M_{2}=M_{1}^{*}$. After this, the remainder of this chapter focuses on recognition problems for matroidal connectivity functions. In Chapter 4 we provided an algorithm to decide when a connectivity function is graphic. Recall that when we refer to a polynomial number of evaluations of the connectivity function, we mean polynomial in the size of the set on which the connectivity function is based. The next step is to attempt to do the same for matroids, which leads to the following question: Is there a polynomial $p$ such that we can identify when a connectivity function, $\lambda$, is the connectivity function of a matroid in at most $p(n)$ evaluations of the connectivity function where $n$ is the size of the set on which $\lambda$ is based? We study this question in Section 5.3 and use spikes, a class of matroids known to provide counterexamples to many natural conjectures, to prove that the answer to this question is no, we cannot identify when a connectivity function is matroidal in a polynomial number of evaluations of the connectivity function.

This, however, is not the only recognition problem for connectivity functions; there is also the question of whether it is possible to add an extra axiom, or axioms, to the axioms of connectivity functions to give an axiomatization of matroidal connectivity functions. In this case we are not looking for something that may be checked in a small number of evaluations of the connectivity function, but rather for something that can be written nicely as an axiom. Mayhew, Newman, and Whittle investigate a similar question for representable matroids. In [7] they investigate whether it is possible to add finitely many axioms to the matroid axioms to give an axiomatization of matroid representability. A discussion on what we mean by written nicely as an axiom can be found in [7]. This leads us to another question:

Open Question 3: Is there an axiom that can be written in a logic that is no stronger than the logic used to write connectivity function axioms, that can be added to the axioms of connectivity functions, to ensure that the connectivity function is the connectivity function of a matroid.

In Section 5.4 we investigate Open Question 3 for the restricted class of self-dual matroids. In particular we find an axiomatization for when a connectivity function comes from an identically self-dual matroid. Unfortunately this didn't seem to give me any intuition about whether or not we can do a similar thing for general matroids. 


\subsection{Matroids With The Same Connectivity Function}

It is proved in [6] that if two matroids, $M_{1}$ and $M_{2}$ have the same connectivity function and $r\left(M_{1}\right) \neq r\left(M_{2}\right)$, then $M_{2}=M_{1}^{*}$ (when $M_{1}^{*}$ is used to denote the matroid dual of $M_{1}$ ). As this is an important result we give a proof of it again here. Our proof is very similar to the one in [6], but uses more elementary results (for instance we do not rely on results of Tutte), and is a little more detailed. The duals in this section will all be matroid duals.

Definition 5.2. Let $M_{1}, M_{2}, M_{3}, M_{4}$ be matroids on a set $E$, where each $M_{i}$, for $i \in\{1,2,3,4\}$, has rank function $r_{i}$. If, for every $X \subseteq E$, we have $r_{1}(X)+r_{2}(X)=r_{3}(X)+r_{4}(X)$, then we say that $\left(M_{1}, M_{2}\right) \sim\left(M_{3}, M_{4}\right)$.

We are interested in such matroids as matroids $M$ and $N$ have the same connectivity function if, and only if,

$$
\begin{aligned}
& r_{M}(X)+r_{M}(E-X)-r_{M}(E)=r_{N}(X)+r_{N}(E-X)-r_{N}(E) \\
& \quad \Longleftrightarrow r_{M}(X)+r_{M}^{*}(X)-|X|=r_{N}(X)+r_{N}^{*}(X)-|X| \\
& \Longleftrightarrow r_{M}(X)+r_{M}^{*}(X)=r_{N}(X)+r_{N}^{*}(X) \\
& \Longleftrightarrow\left(M, M^{*}\right) \sim\left(N, N^{*}\right) .
\end{aligned}
$$

Definition 5.3. Recall from chapter 2 that we may say that a matroid on a set $E$ is not connected, or disconnected, if the connectivity of some $X \subsetneq E$ such that $X \neq \emptyset$, is equal to 0 . Otherwise we say that the matroid is connected. We say that a pair, $\left(M_{1}, M_{2}\right)$, is connected if $\mu_{M_{1}}(X)+\mu_{M_{2}}(X) \geq 1$ for some $X \subsetneq E$ such that $X \neq \emptyset$.

For $\left(M_{1}, M_{2}\right)$ to be connected it is sufficient that one of $M_{1}$ and $M_{2}$ is connected, however, this is not necessary.

The main theorem for this section is the following:

Theorem 5.4. Let $M_{1}, M_{2}, M_{3}, M_{4}$ be matroids on a set $E$. If $\left(M_{1}, M_{2}\right) \sim$ $\left(M_{3}, M_{4}\right)$ and $\left(M_{1}, M_{2}\right)$ is connected, then $B$ is a base of $M_{1}$ or $M_{2}$ if, and only if, $B$ is a base of $M_{3}$ or $M_{4}$.

It is a well known result, and can be found in [9], that if $B_{i}$ is a basis for matroid $M_{i}$, then $\left|B_{i}\right|=r\left(M_{i}\right)$, so by Theorem 5.4, we are able to say 
that if $\left(M_{1}, M_{2}\right) \sim\left(M_{3}, M_{4}\right)$ and $\left(M_{1}, M_{2}\right)$ is connected, then $\left\{M_{1}, M_{2}\right\}=$ $\left\{M_{3}, M_{4}\right\}$ or $r_{M_{1}}=r_{M_{2}}=r_{M_{3}}=r_{M_{4}}$. Suppose that we have a connected matroid with $r(M) \neq r^{*}(M)$, and a matroid $N$ with the same connectivity function as $M$. Then $\left(M, M^{*}\right) \sim\left(N, N^{*}\right)$ and so $\left\{M, M^{*}\right\}=\left\{N, N^{*}\right\}$.

The following lemma shall be used throughout the remainder of this section.

Lemma 5.5. If $\left(M_{1}, M_{2}\right) \sim\left(M_{3}, M_{4}\right)$ and $e \in E$ then

1. $\left(M_{1} \backslash e, M_{2} \backslash e\right) \sim\left(M_{3} \backslash e, M_{4} \backslash e\right)$

2. $\left(M_{1} / e, M_{2} / e\right) \sim\left(M_{3} / e, M_{4} / e\right)$

3. $\left(M_{1}^{*}, M_{2}^{*}\right) \sim\left(M_{3}^{*}, M_{4}^{*}\right)$

Throughout the remainder of this section we will let $M_{1}, M_{2}, M_{3}, M_{4}$ be matroids on groundset $E$ such that $\left(M_{1}, M_{2}\right) \sim\left(M_{3}, M_{4}\right)$.

Establishing when $\left(M_{1}, M_{2}\right) \sim\left(M_{3}, M_{4}\right)$

The next few lemmas prove that $\left(M_{1}, M_{2}\right) \sim\left(M_{3}, M_{4}\right)$ if, and only if, for all $C \subseteq E$

$\mid\left\{i: C\right.$ is a circuit of $M_{i}$ and $\left.i \in\{1,2\}\right\} \mid=$

$\mid\left\{i: C\right.$ is a circuit of $M_{i}$ and $\left.i \in\{3,4\}\right\} \mid$.

Definition 5.6. A modular pair of circuits of a matroid $M$ is a pair of circuits, $\left\{C_{1}, C_{2}\right\}$, such that $r\left(C_{1} \cup C_{2}\right)=\left|C_{1} \cup C_{2}\right|-2$.

Definition 5.7. Let $C_{1}$ and $C_{2}$ be circuits of $M$. A path joining $C_{1}$ to $C_{2}$ is a sequence of circuits, $P_{0}, \ldots, P_{n}$, of $M$ such that all the following hold:

i) $C_{1}=P_{0}$ and $C_{2}=P_{n}$

ii) $P_{i} \cap P_{i+1} \neq \emptyset$, and

iii) $\left\{P_{i}, P_{i+1}\right\}$ is a modular pair of circuits of $M$ for every $i$.

Lemma 5.8. Suppose that $C_{1}$ and $C_{2}$ are circuits of a matroid $M$, and $e \in C_{1} \cap C_{2}$. If $\left(C_{1} \cup C_{2}\right)-\{e\}$ contains a unique circuit, then $r\left(\left(C_{1} \cup\right.\right.$ $\left.\left.C_{2}\right)-\{e\}\right)=\left|\left(C_{1} \cup C_{2}\right)-\{e\}\right|-1$. Moreover $\left\{C_{1}, C_{2}\right\}$ are a modular pair of circuits. 
Proof. If $e \in C_{1} \cap C_{2}$, then there is some circuit contained in $\left(C_{1} \cup C_{2}\right)-\{e\}$, and so $r\left(\left(C_{1} \cup C_{2}\right)-\{e\}\right)<\left|\left(C_{1} \cup C_{2}\right)-\{e\}\right|$. Suppose that $r\left(\left(C_{1} \cup C_{2}\right)-\{e\}\right)<$ $\left|\left(C_{1} \cup C_{2}\right)-\{e\}\right|-1$, then $\left(C_{1} \cup C_{2}\right)-\{e\}$ contains no independent set of size $\left|\left(C_{1} \cup C_{2}\right)-\{e\}\right|-1$. Therefore $\left(\left(C_{1} \cup C_{2}\right)-\{e\}\right)-\{f\}$ must be dependent for all $f \in C_{1} \cup C_{2}$, so for some $\alpha \in C_{1}$ it must be that $\left(\left(C_{1} \cup C_{2}\right)-\{e\}\right)-\{\alpha\}$ contains some circuit $C_{3}$, and, as $C_{3}$ cannot be contained in $C_{1}$ it must be that $C_{3}$ contains some $\beta$ where $\beta \in C_{2}$ (also note that $C_{3}$ must contain an element of $C_{1}$ as $\left.C_{3} \nsubseteq C_{2}\right)$. We also know that $\left(C_{1} \cup C_{2}\right)-\{e\}-\{\beta\}$ contains a circuit, and this circuit does not contain $\beta$ so is not equal to $C_{3}$. Therefore we have found two distinct circuits contained in $\left(C_{1} \cup C_{2}\right)-\{e\}$.

As $e \in \operatorname{cl}\left(C_{1} \cup C_{2}\right)-\{e\}$ we know that $r\left(C_{1} \cup C_{2}\right)=r\left(\left(C_{1} \cup C_{2}\right)-\{e\}\right)$, so if $\left(C_{1} \cup C_{2}\right)-\{e\}$ contains a unique circuit, then $r\left(C_{1} \cup C_{2}\right)=\left|C_{1} \cup C_{2}\right|-2$, so $\left\{C_{1}, C_{2}\right\}$ are a modular pair of circuits.

The following lemma is a direct consequence of a result of Tutte given in [14]. However, we give a proof here which does not use that result. We do however need the strong circuit elimination axiom, which can be found in [9], that is: Let $M$ be a matroid with set of circuits $\mathcal{C}$. If $C_{1}$ and $C_{2} \in \mathcal{C}$ are such that $e \in C_{1} \cap C_{2}$ and $f \in C_{1}-C_{2}$, then there is some $C_{3} \in \mathcal{C}$ such that $f \in C_{3} \subseteq\left(C_{1} \cup C_{2}\right)-\{e\}$.

We use $A \triangle B$ to denote the symmetric difference of $A$ and $B$ (ie $(A-B) \cup$ $(B-A))$.

Lemma 5.9. Let $C_{1}$ and $C_{2}$ be circuits of a matroid $M$ with $e \in C_{1} \cap C_{2}$. Then there is a path, $P_{1}, \ldots, P_{n}$, from $C_{1}$ to $C_{2}$ such that $e \in P_{i}$ for all $i \in\{1, \ldots, n\}$.

Proof. We prove this result by induction on $\left|C_{1} \cup C_{2}\right|$. For the base case assume that $\left|C_{1} \cup C_{2}\right|=\left|C_{1}\right|+1$. Suppose that $\left(C_{1} \cup C_{2}\right)-\{e\}$ contains at least two distinct circuits. As these two circuits cannot be contained in $C_{1}-\{e\}$ they must both contain the element, $\beta$ in $C_{2}-C_{1}$. We shall refer to these two circuits as $C_{3}$ and $C_{4}$. By the circuit elimination axiom this means that there is a circuit in $\left(C_{3} \cup C_{4}\right)-\{\beta\}$ which, as $e \notin C_{3} \cup C_{4}$, is a subset of, but is not equal to, $C_{1}$. This is a contradiction as $C_{1}$ is a circuit. Therefore there is a unique circuit contained in $\left(C_{1} \cup C_{2}\right)-\{e\}$ and so, by Lemma 5.8 . $r\left(C_{1} \cup C_{2}\right)=\left|C_{1} \cup C_{2}\right|-2$. 
Suppose that $C_{1}$ and $C_{2}$ are circuits and $e \in C_{1} \cap C_{2}$. Then, by the circuit elimination axiom, there exists some $C_{3} \subseteq\left(C_{1} \cup C_{2}\right)-\{e\}$. Suppose that there is some such $C_{3}$ such that there is some $\alpha \in C_{1} \triangle C_{2}$ such that $\alpha \notin C_{3}$. Without loss of generality we shall assume that $\alpha \in C_{1}-C_{2}$. As $C_{3} \nsubseteq C_{1}$ there is some $\beta \in\left(C_{2}-C_{1}\right) \cap C_{3}$. By the strong circuit elimination axiom, as $e \in C_{2}-C_{3}$, this means that we can find some $C_{4} \subseteq\left(C_{3} \cup C_{2}\right)-\{\beta\}$ such that $e \in C_{4}$, and $\left|C_{1} \cup C_{4}\right|<\left|C_{1} \cup C_{2}\right|$ and $\left|C_{2} \cup C_{4}\right|<\left|C_{1} \cup C_{2}\right|$. Therefore, by the induction hypothesis there is a path from $C_{1}$ to $C_{4}$ and from $C_{4}$ to $C_{2}$, and so there is a path form $C_{1}$ to $C_{2}$.

Suppose there is no $\alpha \in C_{1} \triangle C_{2}$ such that $\alpha \notin C_{3}$. If there is only one circuit in $\left(C_{1} \cup C_{2}\right)-\{e\}$ then $r\left(C_{1} \cup C_{2}\right)=\left|C_{1} \cup C_{2}\right|-2$ and so we are done. Therefore we shall assume that there are two circuits, $C_{3}$ and $C_{4}$ in $\left(C_{1} \cup C_{2}\right)-\{e\}$. For all $\alpha \in C_{1} \triangle C_{2}$, by our assumption $\alpha \in C_{3} \cap C_{4}$. Without loss of generality let $\alpha \in C_{1}-C_{2}$. Then by the circuit elimination axiom there is some $C_{5} \subseteq\left(C_{3} \cup C_{4}\right)-\{\alpha\}$ such that $e \notin C_{5}$. Therefore $e \in C_{2}-C_{5}$ and there is some $\beta \in C_{2}$ that is also in $C_{5}$, as otherwise $C_{5} \subsetneq C_{1}$. By the strong circuit elimination axiom this means that there is some $C_{6} \subseteq\left(C_{5} \cup C_{2}\right)-\{\beta\}$ such that $e \in C_{6}$ (note that this does not contain $\alpha$ so is not $\left.C_{1}\right)$. As $C_{6} \subseteq C_{1} \cup C_{2}$ and contains neither $\alpha$ nor $\beta$ this means that $\left|C_{1} \cup C_{6}\right|<\left|C_{1} \cup C_{2}\right|$ and $\left|C_{2} \cup C_{6}\right|<\left|C_{1} \cup C_{2}\right|$. Therefore, by the induction hypothesis there is a path from $C_{1}$ to $C_{6}$ and from $C_{6}$ to $C_{2}$, and so there is a path form $C_{1}$ to $C_{2}$.

Lemma 5.10. Let $\left\{C_{1}, C_{2}\right\}$ be a modular pair of circuits in a matroid, and let $\alpha \in C_{1}-C_{2}$. Then $C_{2}$ is the unique circuit contained in $C_{1} \cup C_{2}-\{\alpha\}$

Proof. Let $\alpha \in C_{1}-C_{2}$ and $\beta \in C_{2}-C_{1}$ be arbitrary. Then, as $C_{1}$ and $C_{2}$ are circuits, we have $\alpha, \beta \in \operatorname{cl}\left(\left(C_{1}-\{\alpha\}\right) \cup\left(C_{2}-\{\beta\}\right)\right)$, so $r\left(\left(C_{1}-\{\alpha\}\right) \cup\right.$ $\left.\left(C_{2}-\{\beta\}\right)\right)=r\left(C_{1} \cup C_{2}\right)=\left|C_{1} \cup C_{2}\right|-2$. Therefore, as $\mid\left(C_{1}-\{\alpha\}\right) \cup$ $\left(C_{2}-\{\beta\}\right)|=| C_{1} \cup C_{2} \mid-2=r\left(\left(C_{1}-\{\alpha\}\right) \cup\left(C_{2}-\{\beta\}\right)\right)$, it must be that $\left(C_{1}-\{\alpha\}\right) \cup\left(C_{2}-\{\beta\}\right)$ is independent.

Suppose there is some circuit, $C \subseteq C_{1} \cup C_{2}-\{\alpha\}$. Then, if there were some $\beta \in C_{2}-C_{1}$ such that $\beta \notin C, C$ would be a circuit contained in $\left(C_{1}-\{\alpha\}\right) \cup$ $\left(C_{2}-\{\beta\}\right)$, which would contradict the fact that $\left(C_{1}-\{\alpha\}\right) \cup\left(C_{2}-\{\beta\}\right)$ is independent. Therefore every $\beta \in C_{2}-C_{1}$ is contained in $C$. Suppose that $C \neq C_{2}$, Then, for any $\beta \in C_{2}-C_{1}$, there exists some $C_{3} \subseteq C \cup C_{2}-\beta$. 
This means that $C_{3} \subseteq\left(\left(C_{1}-\{\alpha\}\right) \cup\left(C_{2}-\{\beta\}\right)\right)$, which is a contradiction as $\left(C_{1}-\{\alpha\}\right) \cup\left(C_{2}-\{\beta\}\right)$ is independent. Therefore no such $C_{3}$ exists, so $C=C_{2}$.

Lemma 5.11. Let $\left\{C_{1}, C_{2}\right\}$ be a modular pair of circuits and let e $\in C_{1} \cap C_{2}$. Then for every $C \subseteq\left(C_{1} \cup C_{2}\right)-\{e\}$, the symmetric difference, $C_{1} \triangle C_{2}$ is contained in $C$. Moreover $\left\{C, C_{1}\right\}$ and $\left\{C, C_{2}\right\}$ are modular pairs of circuits.

Proof. Suppose that there is some $C \subseteq\left(C_{1} \cup C_{2}\right)-\{e\}$ such that for some $\alpha \in C_{1} \triangle C_{2}$, we have $\alpha \notin C$. Without loss of generality we shall assume that $\alpha \in C_{1}-C_{2}$. As both $C$ and $C_{1}$ are distinct circuits, $C \nsubseteq C_{1}$, so there is some $\beta \in C_{2}-C_{1}$ such that $\beta \in C$. Therefore $\beta \in C_{2} \cap C$. Therefore, by circuit elimination, there is some $C_{3} \subseteq C_{2} \cup C-\{\beta\} \subseteq C_{1} \cup C_{2}-\{\alpha, \beta\}$ so $C_{3} \neq C_{2}$ and $C_{3}$ does not contain $\alpha$. This contradicts Lemma 5.10, so there can be no $\alpha \in C_{1} \triangle C_{2}$ such that $\alpha \notin C$.

Now consider $r\left(C \cup C_{i}\right)$ for $i \in\{1,2\}$. As $C_{1} \triangle C_{2} \subseteq C$, it must be that $r\left(C \cup C_{i}\right)=r\left(C_{1} \cup C_{2}\right)=\left|C_{1} \cup C_{2}\right|-2=\left|C \cup C_{i}\right|-2$.

Lemma 5.12. Suppose that

$\mid\left\{i: C\right.$ is a circuit of $M_{i}$ and $\left.i \in\{1,2\}\right\} \mid=$

$\mid\left\{i: C\right.$ is a circuit of $M_{i}$ and $\left.i \in\{3,4\}\right\} \mid$,

for all $C \subseteq E$. Then $\left(M_{1}, M_{2}\right) \sim\left(M_{3}, M_{4}\right)$.

Proof. The result holds when $|E|=1$.

Assume, for the purposes of induction, that $\left(M_{1} \backslash e, M_{2} \backslash e\right) \sim\left(M_{3} \backslash e, M_{4} \backslash e\right)$ for every $e \in E$. We need to show that $\left(M_{1}, M_{2}\right) \sim\left(M_{3}, M_{4}\right)$. To do this it suffices to show that $r\left(M_{1}\right)+r\left(M_{2}\right)=r\left(M_{3}\right)+r\left(M_{4}\right)$, as $\left(M_{1} \backslash e, M_{2} \backslash e\right) \sim$ $\left(M_{3} \backslash e, M_{4} \backslash e\right)$ for every $e \in E$. Suppose that $r\left(M_{1}\right)+r\left(M_{2}\right) \neq r\left(M_{3}\right)+r\left(M_{4}\right)$. Then, as $r\left(M_{1} \backslash e\right)+r\left(M_{2} \backslash e\right)=r\left(M_{3} \backslash e\right)+r\left(M_{4} \backslash e\right)$ by the induction hypothesis, and $r\left(M_{1}\right)+r\left(M_{2}\right) \neq r\left(M_{3}\right)+r\left(M_{4}\right)$ by assumption, we must have:

$r\left(M_{1}\right)-r\left(M_{1} \backslash e\right)+r\left(M_{2}\right)-r\left(M_{2} \backslash e\right) \neq r\left(M_{3}\right)-r\left(M_{3} \backslash e\right)+r\left(M_{4}\right)-r\left(M_{4} \backslash e\right)$.

If $E-\{e\}$ spanned $e$ in all $M_{i}$ then $r_{i}(E)=r_{i}(E-\{e\})$ for all $i$ and so we would have $r\left(M_{1}\right)-r\left(M_{1} \backslash e\right)+r\left(M_{2}\right)-r\left(M_{2} \backslash e\right)=r\left(M_{3}\right)-r\left(M_{3} \backslash\right.$ 
$e)+r\left(M_{4}\right)-r\left(M_{4} \backslash e\right)$, a contradiction. Therefore there is some $i$ such that $E-\{e\}$ does not span $e$ in $M_{i}$. This means that $e$ is a coloop of some $M_{i}$. Without loss of generality let $i=1$, in other words, suppose that $e$ is a coloop of $M_{1}$.

Claim 5.12.1. $E-\{e\}$ spans e in $M_{i}$ for all $i \in\{2,3,4\}$.

Proof. First suppose that $E-\{e\}$ does not span $e$ in $M_{2}$. This means that $e$ is a coloop of $M_{2}$. Therefore $e$ is not contained in any circuit of $M_{2}$ and, as $e$ is also a coloop of $M_{1}, e$ is not contained in any circuit of $M_{1}$. By the assumption in the statement of the theorem this means that $E-\{e\}$ does not span $e$ in $M_{3}$ or in $M_{4}$. Therefore $r_{i}(E)=r_{i}(E-\{e\})+1$, so $r\left(M_{1}\right)+r\left(M_{1} \backslash e\right)+r\left(M_{2}\right)-r\left(M_{2} \backslash e\right)=r\left(M_{3}\right)-r\left(M_{3} \backslash e\right)+r\left(M_{4}\right)-$ $r\left(M_{4} \backslash e\right)$, a contradiction. Therefore $E-\{e\}$ must span $e$ in $M_{2}$.

It cannot be that $E-\{e\}$ spans $e$ in exactly one of $M_{3}$ and $M_{4}$ as otherwise we would have $r\left(M_{1}\right)-r\left(M_{1} \backslash e\right)+r\left(M_{2}\right)-r\left(M_{2} \backslash e\right)=$ $r\left(M_{3}\right)-r\left(M_{3} \backslash e\right)+r\left(M_{4}\right)-r\left(M_{4} \backslash e\right)$, a contradiction. Therefore $E-\{e\}$ either spans $e$ in neither or both of $M_{3}$ and $M_{4}$. Suppose that $E-\{e\}$ spans $e$ in neither $M_{3}$ nor $M_{4}$. This would make $e$ a coloop of $M_{3}$ and of $M_{4}$ so $e$ would not be contained in any circuit of $M_{3}$ or of $M_{4}$. However, this contradicts the assumption in the statement of the theorem as $E-\{e\}$ spans $e$ in $M_{2}$ and so $e$ is contained in some circuit of $M_{2}$. Therefore $E-\{e\}$ must span both $M_{3}$ and $M_{4}$.

This means that every circuit, $C$, of $M_{3}$ or $M_{4}$ which contains $e$, must also be a circuit of $M_{2}$, and $C$ can only be a circuit of exactly one of $M_{3}$ and $M_{4}$ (as $M_{1}$ does not contain any circuit containing $e$ ). Let $C_{3}$ be a circuit of $M_{3}$ which contains $e$ and $C_{4}$ be a circuit of $M_{4}$ which also contains $e$. Note that $C_{3}$ and $C_{4}$ are also circuits of $M_{2}$. Therefore by Lemma 5.9, there is a path, $P_{0}, \ldots, P_{n}$ in $M_{2}$, such that $P_{0}=C_{3}$ and $P_{n}=C_{4}$, and each $P_{i}$ contains $e$. Therefore $P_{i}$ is not a circuit in $M_{1}$ for all $i \in\{0, \ldots, n\}$, so each $P_{i}$ is a circuit in exactly one of $M_{3}$ and $M_{4}$. Therefore there is some $P_{k}$ so that $P_{k}$ is a circuit in $M_{3}$, and $P_{k+1}$ is a circuit in $M_{4}$. Let $C$ be the circuit of $M_{2}$ contained in $P_{k} \cup P_{k+1}-\{e\}$ (such a circuit exists by the circuit exchange axiom). As $C$ is a circuit of $M_{2}$ it must also be a circuit of $M_{3}$ or $M_{4}$. 
Without loss of generality say that $C$ is a circuit of $M_{3}$.

Let $\alpha \in P_{k}-P_{k+1}$. Then, by Lemma 5.11, $\alpha \in C$ and $\left\{C, P_{k}\right\}$ is a modular pair of circuits.

Let $C^{\prime}$ be a circuit of $M_{3}$ such that $e \in C^{\prime}$ and $C^{\prime} \subseteq\left(P_{k} \cup C\right)-\{\alpha\}$. There will be such a circuit by the strong circuit elimination axiom as both $P_{k}$ and $C$ are circuits in $M_{3}$. By Lemma 5.10, as $\left\{C, P_{k}\right\}$ is a modular pair of circuits, $C^{\prime}=P_{k+1}$

This means that $P_{k+1}$ is a circuit in both $M_{3}$ and $M_{4}$, a contradiction.

Let $M$ be a matroid with circuit set $\mathcal{C}$. Recall that the collection of circuits of $M \backslash\{e\}$ is $\{C \mid C \in \mathcal{C}, e \notin C\}$

Lemma 5.13. Suppose that $\left(M_{1}, M_{2}\right) \sim\left(M_{3}, M_{4}\right)$. If $C \subseteq E$ then

$\mid\left\{i: C\right.$ is a circuit of $M_{i}$ and $\left.i \in\{1,2\}\right\} \mid=$

$\mid\left\{i: C\right.$ is a circuit of $M_{i}$ and $\left.i \in\{3,4\}\right\} \mid$.

Proof. Suppose that we can find some $M_{1}, M_{2}, M_{3}, M_{4}, E$ and $C$ for which the lemma fails. Choose these such that $|C|+|E|$ is as small as possible.

Claim 5.13.1. $E=C$.

Proof. Suppose $E \neq C$. Then there is some $e \in E-C$. By Lemma 5.5, $\left(M_{1} \backslash\{e\}, M_{2} \backslash\{e\}\right) \sim\left(M_{3} \backslash\{e\}, M_{4} \backslash\{e\}\right)$, and, as $e \notin C, C$ is a circuit of $M_{i} \backslash\{e\}$ if, and only if, $C$ is a circuit of $M$ for $i \in\{1,2,3,4\}$. Therefore $\mid\left\{i: C\right.$ is a circuit of $M_{i} \backslash\{e\}$ and $\left.i \in\{1,2\}\right\} \mid$

$\neq \mid\left\{i: C\right.$ is a circuit of $M_{i} \backslash\{e\}$ and $\left.i \in\{3,4\}\right\} \mid$, contradicting the minimality of $|E|+|C|$.

$C$ cannot be a circuit of both $M_{1}$ and either $M_{3}$ or $M_{4}$. Without loss of generality suppose that $C$ was a circuit of both $M_{1}$ and $M_{3}$. This would mean $M_{1}=M_{3}$ and so, since $\left(M_{1}, M_{2}\right) \sim\left(M_{3}, M_{4}\right)$, we would have $M_{2}=M_{4}$, which would mean $\mid\left\{i: C\right.$ is a circuit of $M_{i} \backslash\{e\}$ and $\left.i \in\{1,2\}\right\} \mid$

$=\mid\left\{i: C\right.$ is a circuit of $M_{i} \backslash\{e\}$ and $\left.i \in\{3,4\}\right\} \mid$, a contradiction. Therefore $C$ cannot be a circuit of both $M_{1}$ and $M_{3}$ or $M_{4}$.

Suppose $C$ were a circuit of $M_{1}$ and $M_{2}$. By the previous paragraph $C$ cannot be a circuit of either $M_{3}$ or $M_{4}$. 
Claim 5.13.2. Neither $M_{3}$ nor $M_{4}$ contains a circuit.

Proof. Suppose that $M_{3}$ contained a circuit, $C^{\prime}$. As $C^{\prime} \neq C$ there is some $e \in E$ that is not in $C^{\prime}$. This means that $C^{\prime}$ is also a circuit of $M_{3} \backslash\{e\}$, but is not a circuit of $M_{1} \backslash\{e\}$ or $M_{2} \backslash\{e\}$ (as $C$ is a circuit of both $M_{1}$ and $\left.M_{2}\right)$. Therefore $\mid\left\{i: C\right.$ is a circuit of $M_{i} \backslash\{e\}$ and $\left.i \in\{1,2\}\right\} \mid$

$=\mid\left\{i: C\right.$ is a circuit of $M_{i} \backslash\{e\}$ and $\left.i \in\{3,4\}\right\} \mid$, and $\left(M_{1} \backslash\{e\}, M_{2} \backslash\right.$ $\{e\}) \sim\left(M_{3} \backslash\{e\}, M_{4} \backslash\{e\}\right)$, contradicting the minimality of $|C|+|E|$.

Therefore $r\left(M_{1}\right)+r\left(M_{2}\right)=2|E|-2 \neq 2|E|=r\left(M_{3}\right)+r\left(M_{4}\right)$ and so $\left(M_{1}, M_{2}\right) \nsim\left(M_{3}, M_{4}\right)$; a contradiction.

Suppose that $C$ is a circuit of $M_{1}$ but not of $M_{i}$ for $i \in\{2,3,4\}$. Let $C^{\prime} \subsetneq E$. If $C^{\prime}$ is a circuit of $M_{3}$ or $M_{4}$ then $C^{\prime}$ is a circuit of $M_{2}$ and exactly one of $M_{3}$ or $M_{4}$ (as otherwise $|C|+|E|$ would not be as small as possible). Let $N$ be a matroid on groundset $E$ with no circuits. Then $\mid\left\{i: C\right.$ is a circuit of $N_{i} \backslash\{e\}$ and $\left.i \in\{1,2\}\right\} \mid$

$=\mid\left\{i: C\right.$ is a circuit of $N_{i} \backslash\{e\}$ and $\left.i \in\{3,4\}\right\} \mid$, where $N_{1}=N$ and $N_{i}=M_{i}$ for all $i \in\{2,3,4\}$; which, by Lemma 5.12 , means that $\left(N, M_{2}\right) \sim$ $\left(M_{3}, M_{4}\right)$. But $r(N)=r\left(M_{1}\right)+1$ so this contradicts the assumption given in the lemma, that $\left(M_{1}, M_{2}\right) \sim\left(M_{3}, M_{4}\right)$.

\section{Matroids with Low Connectivity}

For this section we shall suppose that $M_{1}, M_{2}, M_{3}, M_{4}$ are matroids on a set $E$ such that $\left(M_{1}, M_{2}\right) \sim\left(M_{3}, M_{4}\right)$, and that $M_{i}$ has connectivity function $\mu_{M_{i}}$ for $i \in\{1,2,3,4\}$. We shall further suppose that $M_{1}$ and $M_{2}$ are such that $1 \leq \mu_{M_{1}}(X)+\mu_{M_{2}}(X) \leq 2$ for all $X \subsetneq E$ such that $X \neq \emptyset$. We study these cases separately as these are the pairs of matroids that are connected but may have $\left(M_{1} \backslash\{e\}, M_{2} \backslash\{e\}\right)$ not connected for some $e \in E$.

We use $M \mid A$ to denote $M \backslash(E-A)$.

We shall also need the following Lemma which can be found in [9].

Lemma 5.14. Suppose that $M$ and $N$ are connected matroids on the same groundset, E. If $e \in E$ and $M_{1}$ and $M_{2}$ have the same circuits containing e, then $M_{1}=M_{2}$. 
Lemma 5.15. If there is an $X$ that is a connected component in $M_{1}$ and $M_{2}$ and is such that $M_{1}\left|X=M_{2}\right| X$, then $M_{1}=M_{3}$

Proof. Choose $A_{1}, A_{2} \subseteq E$ such that all the following hold:

i) $\mu_{i}\left(A_{i}\right)=0$ for $i \in\{1,2\}$,

ii) $M_{i}\left|A_{i}=M_{i+2}\right| A_{i}$,

iii) $\left|A_{1}\right|+\left|A_{2}\right|$ is a maximum.

We know that we can find some such $A_{1}$ and $A_{2}$, as choosing $A_{2}=\emptyset$ and $A_{1}=N$, where $N$ is the connected component common to both $M_{1}$ and $M_{3}$, will give a pair $A_{1}$ and $A_{2}$ that fulfil i)-iii) above. This means that we cannot have both $A_{1}$ and $A_{2}=\emptyset$, as we can find some $A_{1} \neq \emptyset$ that works, and we require $\left|A_{1}\right|+\left|A_{2}\right|$ to be a maximum.

Suppose that $A_{1}=A_{2}$. As $\left(M_{1}, M_{2}\right)$ is connected, then $\mu_{M_{1}}\left(A_{1}\right)+\mu_{M_{2}}\left(A_{1}\right)>$ 0 unless $A_{1}=\emptyset$ or $E$. As we know that $\mu_{M_{1}}\left(A_{1}\right)=0$ and $\mu_{M_{2}}\left(A_{1}\right)=$ $\mu_{M_{2}}\left(A_{2}\right)=0$, either $A_{1}$ or $A_{2}=\emptyset$ or $E$. As $A_{1} \cup A_{2} \neq \emptyset$, this means that $A_{1}=A_{2}=E$ and so $M_{1}=M_{2}$.

Suppose that there is some $e \in A_{1}-A_{2}$ (a similar argument works if $e \in$ $\left.A_{2}-A_{1}\right)$. As $e \in A_{1}$ and $M_{1}\left|A_{1}=M_{3}\right| A_{1}$, we know that $M_{1}$ and $M_{3}$ contain the same circuits containing e. By Lemma 5.14, we know that this means that the connected component of $M_{1}$ containing $e$, which we shall call $N_{1}$, and the connected component of $M_{3}$ containing $e$, which we shall call $N_{3}$, are equal. As $\left(M_{1}, M_{2}\right) \sim\left(M_{3}, M_{4}\right)$, by Lemma 5.13 this means that $M_{2}$ and $M_{4}$ have the same circuits containing $e$ and so the connected component of $M_{2}$ which contains $e$, which we shall call $N_{2}$ and the connected component of $M_{4}$ which contains $e$, which we shall call $N_{4}$, are equal. As $e \in A_{1}-A_{2}$, we know that $A_{2} \neq N_{2}$, but

i) $\mu_{M_{2}}\left(A_{2} \cup E\left(N_{2}\right)\right)=0$,

ii) $\mu_{M_{2} \mid\left(A_{2} \cup E\left(N_{2}\right)\right)}=\mu_{M_{4} \mid\left(A_{2} \cup E\left(N_{2}\right)\right)}$ as $\mu_{M_{2} \mid A_{2}}=\mu_{M_{4} \mid A_{2}}$ and $\mu_{M_{2} \mid E\left(N_{2}\right)}=$ $\mu_{M_{4} \mid E\left(N_{2}\right)}$.

and $\left|A_{1}\right|+\left|A_{2} \cup E\left(N_{2}\right)\right|>\left|A_{1}\right|+\left|A_{2}\right|$, contradicting the assumption that $\left|A_{1}\right|+\left|A_{2}\right|$ is a maximum. Therefore we must have $A_{1}=A_{2}$, and so the result follows. 
We now consider matroids on $E$ that have a set $X \subsetneq E$ such that $|X|, \mid E-$ $X \mid>1$ and $\mu(X)=1$.

Definition 5.16. Let $M$ and $N$ be matroids, both with at least two elements such that $E(M) \cap E(N)=\{e\}$ where $e$ is neither a loop nor a coloop. Then $M \oplus_{2} N$, known as the two-sum of $M$ and $N$, is the matroid with groundset $(E(M) \cup E(N))-\{e\}$ and collection of circuits:

$\mathcal{C}(M \backslash\{e\})+\mathcal{C}(N \backslash\{e\}) \cup\{(C \cup D)-\{e\}: e \in C \in \mathcal{C}(M)$ and $e \in D \in \mathcal{C}(N)\}$

The following theorem can be found in [10].

Theorem 5.17. Let $M$ be a matroid on groundset $E$ with connectivity function $\mu$, and let $\left(X_{1}, X_{2}\right)$ be a partition of $E$ such that $\mu\left(X_{1}\right)=1$. Then there are matroids $M_{1}$ and $M_{2}$ on $X_{1} \cup\{e\}$ and $X_{2} \cup\{e\}$ respectively where $e \notin E$, such that $M$ is the 2-sum of $M_{1}$ and $M_{2}$. Conversely, if $M$ is the 2-sum of $M_{1}$ and $M_{2}$, then $\mu\left(E\left(M_{1}\right)-E\left(M_{2}\right)\right)=1$ and $M_{1}$ and $M_{2}$ are isomorphic to minors of $M$.

Lemma 5.18. Suppose that $N_{1}$ and $N_{2}$ are matroids on a set $E$ such that for some $X \subsetneq E$ such that $|X|,|E-X|>1$, we have $\mu_{N_{1}}(X)=\mu_{N_{2}}(X)=1$. If $N_{1}$ and $N_{2}$ have the same set of circuits intersecting both $X$ and $E-X$, then $N_{1}$ and $N_{2}$ have a connected component in common.

Proof. As $\mu_{N_{1}}(X)=\mu_{N_{2}}(X)=1$, then by Theorem 5.17 we can write $N_{1}$ as a 2-sum of matroids, $H_{1}$ and $H_{1}^{\prime}$, and $N_{2}$ as a 2-sum of matroids, $H_{2}$ and $H_{2}^{\prime}$, where $E\left(H_{i}\right)=X \cup\{e\}$ for $i \in\{1,2\}$ and $e \notin E$.

As $N_{1}$ and $N_{2}$ have the same circuits intersecting both $X$ and $E-X$, this means, by the definition of 2-sum, that $H_{1}$ and $H_{2}$ have the same circuits containing $e$. Let $J_{1}$ be the connected component of $H_{1}$ that contains $e$ and $J_{2}$ be the connected component of $H_{2}$ that contains $e$. As $J_{1}$ and $J_{2}$ both contain the same circuits containing $e$ and $J_{1}$ and $J_{2}$ are connected, $J_{1}=J_{2}$. Similarly let $J_{1}^{\prime}$ be the connected component of $H_{1}^{\prime}$ that contains $e$ and $J_{2}^{\prime}$ be the connected component of $H_{2}^{\prime}$ that contains $e$. By the reasoning above $H_{1}^{\prime}$ and $H_{2}^{\prime}$ will have the same circuits containing $e$ so $J_{1}^{\prime}=J_{2}^{\prime}$. Therefore $J_{1} \triangle J_{1}^{\prime}=J_{2} \triangle J_{2}^{\prime}$ and $J_{1} \triangle J_{1}^{\prime}$ is a connected component of $N_{1}$ and $J_{2} \triangle J_{2}^{\prime}$ is a connected component of $N_{2}$. 
Lemma 5.19. Suppose that $\left(M_{1}, M_{2}\right) \sim\left(M_{3}, M_{4}\right)$ and there is some $X \subsetneq E$ such that $|X|,|E-X|>1$, that has $\mu_{M_{1}}(X)=\mu_{M_{3}}(X)=1$. Then if $M_{1}$ and $M_{3}$ have the same circuits intersecting both $X$ and $E-X$, we have $M_{1}=M_{3}$.

Proof. This follows immediately from Lemma 5.18 and Lemma 5.15.

Before proving the following lemma we note that if $\left(M_{1}, M_{2}\right) \sim\left(M_{3}, M_{4}\right)$, then $\mu_{M_{1}}(X)+\mu_{M_{2}}(X)=\mu_{M_{3}}(X)+\mu_{M_{4}}(X)$. Recall, from Lemma 2.20, that if $\mu(X)=0$ for some $X \subsetneq E$, then there cannot be a circuit that intersects both $X$ and $E-X$.

Lemma 5.20. If $\mu_{M_{1}}(X)+\mu_{M_{2}}(X)=1$ for some $X \subseteq E$, then $M_{1}=M_{3}$ or $M_{1}=M_{4}$.

Proof. As $\left(M_{1}, M_{2}\right) \sim\left(M_{3}, M_{4}\right)$, we can, without loss of generality, assume that $\mu_{M_{1}}(X)=\mu_{M_{3}}(X)=1$ and $\mu_{M_{2}}(X)=\mu_{M_{4}}(X)=0$.

Suppose that $|X|=1$. Then, as $\mu_{M_{2}}(X)=\mu_{M_{4}}(X)=0$, we have $r_{M_{2 i}}(X)+$ $r_{M_{2 i}}^{*}(X)=|X|$ for $i \in\{1,2\}$, and so either $r_{M_{2 i}}(X)=0$ or $r_{M_{2 i}}^{*}(X)=0$. In other words, $X$ is either a loop or a coloop in $M_{2 i}$. Assume that $X$ is a loop in $M_{2}$. Then, as $\left(M_{1}, M_{2}\right) \sim\left(M_{3}, M_{4}\right)$ it must be that $X$ is loop of $M_{3}$ or $M_{4}$ by Lemma 5.13. In fact $X$ must be a loop of $M_{4}$, as $\mu_{M_{3}}(X)=1$. Therefore, if $|X|=1$ and $X$ is a loop of $M_{2}$, then $X$ is a loop of $M_{4}$. By Lemma 5.5 we can use a similar argument to show that if $|X|=1$ and $X$ is a coloop of $M_{2}$, then $X$ is a coloop of $M_{4}$.

Suppose $|E-X|=1$. Then, by a similar argument, $X$ is either a loop of $M_{2}$ and $M_{4}$, or a coloop of $M_{2}$ and $M_{4}$.

Therefore if $|X|=1$ or $|E-X|=1$, either the matroids $M_{2}$ and $M_{4}$ have a connected component in common, in which case, by Lemma 5.15, $M_{2}=M_{4}$; or $M_{2}^{*}$ and $M_{4}^{*}$ have a connected component in common, in which case, as $\left(M_{1}^{*}, M_{2}^{*}\right) \sim\left(M_{3}^{*}, M_{4}^{*}\right)$ by Lemma 5.5, we have $M_{2}^{*}=M_{4}^{*}$ by Lemma 5.15 . and so $M_{2}=M_{4}$. Therefore we must also have $M_{1}=M_{3}$.

Now suppose that $|X|>1$ and $|E-X|>1$. As $\mu_{M_{2 i}}(X)=0$ for $i \in\{1,2\}$, there is no circuit of $M_{2 i}$ that intersects both $X$ and $E-X$. Therefore, as $\left(M_{1}, M_{2}\right) \sim\left(M_{3}, M_{4}\right)$, the matroids $M_{1}$ and $M_{3}$ must have the same circuits intersecting both $X$ and $E-X$ by Lemma 5.13. The result follows by Lemma 5.19 . 
Lemma 5.21. Let $\mu_{M_{1}}(X)=\mu_{M_{2}}(X)=\mu_{M_{3}}(X)=\mu_{M_{4}}(X)=1$ and let $|X|,|E-X| \geq 2$. Then either $M_{1} \in\left\{M_{3}, M_{4}\right\}$ or there are matroids $N_{i}$ and $N$ for $i \in\{1,2,3,4\}$ such that all the following hold:

1. $\left(N_{1}, N_{2}\right) \sim\left(N_{3}, N_{4}\right)$,

2. $M_{i}=N_{i} \oplus_{2} N$,

3. $E \cap E(N) \in\{X, E-X\}$

Proof. As $\mu_{M_{i}}(X)=1$ for some $X$ such that $|X|,|E-X| \geq 2$, then, by Theorem 5.17, $M_{i}$ is the two-sum of two matroids with $X \cup\{e\}$ and $(E-X) \cup$ $\{e\}$ as their groundsets. Define $C_{i, Z}$, for $Z \in\{X, E-X\}$ and $i \in\{1,2,3,4\}$, as follows:

$C_{i, Z}=\left\{C \cap Z: C\right.$ is a circuit of $M_{i}$ which intersects both $X$ and $\left.E-X\right\}$.

Claim 5.21.1. $\mathcal{C}_{1, Z} \triangle C_{2, Z}=C_{3, Z} \triangle C_{4, Z}$

Proof. Let $Y \in \mathcal{C}_{i, Z}$. As $M_{i}$ is the two-sum of two matroids, $N_{i}$ and $N_{i}^{\prime}$, then $N_{i}$ is isomrphic to a minor of $M_{i}$. Without loss of generality we may assume that $E\left(N_{i}\right)=Z \cup\{e\}$ for some $e \notin E$. Therefore for some $R, S \subseteq E-Z$, we can say $N_{i}=M_{i} \backslash R / S$ and, by the definition of two-sum, $Y \cup\{e\}$ is a circuit of $N_{i}$.

By Lemma 5.5, $\left(M_{1} \backslash R / S, M_{2} \backslash R / S\right) \sim\left(M_{3} \backslash R / S, M_{4} \backslash R / S\right)$.

By Lemma 5.13, it follows that either $Y \cup\{e\}$ is a circuit of exactly one of $M_{1} \backslash R / S$ and $M_{2} \backslash R / S$ and exactly one of $M_{3} \backslash R / S$ and $M_{4} \backslash R / S$, or $Y \cup\{e\}$ is a circuit of all $M_{i} \backslash R / S$ for $i \in\{1,2,3,4\}$.

We now show that if $Y \cup\{e\}$ is a circuit of $M_{j} \backslash R / S$, then $Y \in \mathcal{C}_{j, Z}$ and so $Y$ is a circuit in exactly one of $\mathcal{C}_{1, Z}$ and $\mathcal{C}_{2, Z}$ and exactly one of $\mathcal{C}_{3, Z}$ and $\mathcal{C}_{4, Z}$ or $Y$ is a circuit in all of $\mathcal{C}_{1, Z}, \mathcal{C}_{2, Z}, \mathcal{C}_{3, Z}$, and $\mathcal{C}_{4, Z}$, and therefore $\mathcal{C}_{1, Z} \triangle C_{2, Z}=C_{3, Z} \triangle C_{4, Z}$. Suppose that $Y \cup\{e\}$ is a circuit of $M_{j} \backslash R / S$. Then there is a circuit, $C$, of $M_{j}$ such that $C \cap R=\emptyset$ and $C-S=Y \cup\{e\}$. As $C \cap Z \neq \emptyset$ and $C-Z \neq \emptyset$, we have $Y=C \cap Z$ and so $Y \in \mathcal{C}_{j, Z}$ 
Claim 5.21.2. If $Y \in \mathcal{C}_{1, Z}-\mathcal{C}_{2, Z}$, and $Y \in \mathcal{C}_{3, Z}-\mathcal{C}_{4, Z}$, then $\mathcal{C}_{1,(E-Z)}=$ $\mathcal{C}_{3,(E-Z)}$ and $\mathcal{C}_{2,(E-Z)}=\mathcal{C}_{4,(E-Z)}$.

Proof. Let $Y^{\prime} \in \mathcal{C}_{1,(E-Z)}$, then, by definition of the two-sum, $Y^{\prime} \cup Y$ is a circuit of $M_{1}$ and not a circuit of $M_{2}$ (as $\left.Y \notin \mathcal{C}_{2, Z}\right)$. Therefore, by Lemma 5.13, $Y^{\prime} \cup Y$ is a circuit of exactly one of $M_{3}$ and $M_{4}$. As $Y \notin \mathcal{C}_{4, Z}$, we know $Y^{\prime} \cup Y$ is not a circuit of $M_{4}$, so $Y^{\prime} \cup Y$ is a circuit of $M_{3}$. Therefore $Y^{\prime} \in \mathcal{C}_{3,(E-Z)}$. This shows that $\mathcal{C}_{1,(E-Z)} \subseteq \mathcal{C}_{3,(E-Z)}$. Similarly we can show that $\mathcal{C}_{3,(E-Z)} \subseteq \mathcal{C}_{1,(E-Z)}$, and therefore $\mathcal{C}_{1,(E-Z)}=$ $\mathcal{C}_{3,(E-Z)}$. As $\mathcal{C}_{1, Z} \triangle C_{2, Z}=C_{3, Z} \triangle C_{4, Z}$, this also means that $\mathcal{C}_{2,(E-Z)}=$ $\mathcal{C}_{4,(E-Z)}$.

We now show that either $M_{1} \in\left\{M_{3}, M_{4}\right\}$ or there are matroids $N_{i}$ and $N$ for $i \in\{1,2,3,4\}$ such that all the following hold:

1. $\left(N_{1}, N_{2}\right) \sim\left(N_{3}, N_{4}\right)$,

2. $M_{i}=N_{i} \oplus_{2} N$

3. $E \cap E(N) \in\{X, E-X\}$,

we split into two cases.

Case 1: assume that $\mathcal{C}_{1, Z} \neq \mathcal{C}_{2, Z}$ for both $Z=X$ and $Z=(E-X)$. In this case we show that we always have $M_{1}=M_{3}$. As $\mathcal{C}_{1, Z} \neq \mathcal{C}_{2, Z}$, we can, without loss of generality, assume that there is some $Y \in \mathcal{C}_{1, Z}-\mathcal{C}_{2, Z}$. Then, by Claim 6.5.1, this means that $Y \in \mathcal{C}_{3, Z} \triangle C_{4, Z}$. Without loss of generality we can assume that $Y \in \mathcal{C}_{3, Z}-\mathcal{C}_{4, Z}$. Therefore, by Claim 6.5.2, $\mathcal{C}_{1,(E-Z)}=$ $\mathcal{C}_{3,(E-Z)}$ and $\mathcal{C}_{2,(E-Z)}=\mathcal{C}_{4,(E-Z)}$.

We now show that $\mathcal{C}_{1, Z}=\mathcal{C}_{3, Z}$. By Lemma 5.19, this means that $M_{1}=M_{3}$. To show that $\mathcal{C}_{1, Z}=\mathcal{C}_{3, Z}$, we assume, without loss of generality, that there is some $Y^{\prime} \in \mathcal{C}_{1, E-Z}-\mathcal{C}_{2, E-Z}$. Then, by Claim 6.5.1. $Y^{\prime} \in \mathcal{C}_{3, E-X} \triangle \mathcal{C}_{4, E-X}$. If $Y^{\prime} \in \mathcal{C}_{4, E-Z}$, then, as $\mathcal{C}_{2,(E-Z)}=\mathcal{C}_{4,(E-Z)}$, this means that $Y^{\prime} \in \mathcal{C}_{2}$ so we get a contradiction. Therefore $Y^{\prime} \in \mathcal{C}_{3, E-X}$. This means that $Y^{\prime} \in \mathcal{C}_{3, E-Z}-\mathcal{C}_{4, E-Z}$. Therefore, by Claim 6.5.2, $\mathcal{C}_{1, Z}=\mathcal{C}_{3, Z}$.

Case 2: suppose that $\mathcal{C}_{1, Z}=\mathcal{C}_{2, Z}$ for some $Z \in\{X, E-X\}$. 
Claim 5.21.3. If $\mathcal{C}_{1, Z}=\mathcal{C}_{2, Z}$ for some $Z \in\{X, E-X\}$, then $\mathcal{C}_{1, Z}=$ $\mathcal{C}_{2, Z}=\mathcal{C}_{3, Z}=\mathcal{C}_{4, Z}$

Proof. As $\mathcal{C}_{1, Z} \triangle \mathcal{C}_{2, Z}=\mathcal{C}_{3, Z} \triangle \mathcal{C}_{4, Z}$ and $\mathcal{C}_{1, Z} \triangle \mathcal{C}_{2, Z}=\emptyset$, we must have $\mathcal{C}_{3, Z} \triangle \mathcal{C}_{4, Z}=0$ and so $\mathcal{C}_{3, Z}=\mathcal{C}_{4, Z}$. It remains to show that $\mathcal{C}_{1, Z}=\mathcal{C}_{3, Z}$. To do this first let $Y \in \mathcal{C}_{1, Z}$. Then there is some circuit $C$ of $M_{1}$ that is such that $C \cap X \neq \emptyset$ and $C \cap(E-X) \neq \emptyset$ and $C \cap Z=Y$. By Lemma 5.13, $C$ is a circuit of $M_{3}$ or $M_{4}$ which, as $M_{3}=M_{4}$, means that $C$ is a circuit of $M_{3}$ and so $Y \in \mathcal{C}_{3, Z}$. Therefore $\mathcal{C}_{1, Z} \subseteq C_{3, Z}$. In a similar way $\mathcal{C}_{3, Z} \subseteq C_{1, Z}$ and so $\mathcal{C}_{1, Z}=\mathcal{C}_{3, Z}$.

Therefore there is some $N, N_{i}$ for $i \in\{1,2,3,4\}$ such that $E(N)=Z \cup\{e\}$ and $E\left(N_{i}\right)=(E-Z) \cup\{e\}$ for some $e \notin E$, and $M_{i}=N_{i} \oplus_{2} N$. As $M_{i}=N_{i} \oplus_{2} N$, the collection of circuits, $C_{N_{i}}$ of $N_{i}$ is equal to:

$$
\left\{C: C \subseteq E-Z \text { and } C \text { is a circuit of } M_{i} \text { or } e \in C \text { and } C-\{e\} \in \mathcal{C}_{i,(E-Z)}\right\}
$$

To show that we have $\left(N_{1}, N_{2}\right) \sim\left(N_{3}, N_{4}\right)$, by Lemma 5.12 it suffices to show that $\mid\left\{i: C\right.$ is a circuit of $N_{i}$ and $\left.i \in\{1,2\}\right\}|=|\{i$ : $C$ is a circuit of $N_{i}$ and $\left.i \in\{3,4\}\right\} \mid$.

Let $C \in \mathcal{C}_{N_{i}}$ and $C \in \mathcal{C}_{N_{j}}$ for $i, j \in\{1,2,3,4\}$ and $i \neq j$. Then $C$ cannot be a circuit in $M_{i}$ and in $\mathcal{C}_{j, Z}$ at the same time, as $\mathcal{C}_{1, Z}=\mathcal{C}_{2, Z}=\mathcal{C}_{3, Z}=\mathcal{C}_{4, Z}$ and no circuit of $M_{i}$ is in $C_{i, Z}$.

Let $C \in \mathcal{C}_{N_{1}}$ and $C \in \mathcal{C}_{N_{2}}$. Either $C$ is a circuit of both $M_{1}$ and $M_{2}$ that does not intersect with $E-Z$ or is a circuit of neither $M_{1}$ nor $M_{2}$. Suppose the former. As $\left(M_{1}, M_{2}\right) \sim\left(M_{3}, M_{4}\right)$ this means that $C$ is a circuit of both $M_{3}$ and $M_{4}$ that does not intersect with $E-Z$, which means that $C \in \mathcal{C}_{N_{3}}$ and $C \in \mathcal{C}_{N_{4}}$. If $C$ is a circuit of neither $M_{1}$ nor $M_{2}$, then $C \in \mathcal{C}_{1, Z}$ and $C \in \mathcal{C}_{2, Z}$. Therefore, as $\mathcal{C}_{1, Z}=\mathcal{C}_{2, Z}=\mathcal{C}_{3, Z}=\mathcal{C}_{4, Z}$, we have $C \in \mathcal{C}_{3, Z}$ and $C \in \mathcal{C}_{4, Z}$, and so $C \in \mathcal{C}_{N_{3}}$ and $C \in \mathcal{C}_{N_{4}}$.

Let $C \in \mathcal{C}_{N_{1}}$ and $C \notin \mathcal{C}_{N_{2}}$. We cannot have $C \in C_{i, Z}$ for $i \in\{1,2,3,4\}$ since, as $\mathcal{C}_{1, Z}=\mathcal{C}_{2, Z}=\mathcal{C}_{3, Z}=\mathcal{C}_{4, Z}$, this would mean $C \in \mathcal{C}_{2, Z}$, a contradiction. Therefore $C$ is a circuit of $M_{1}$ and not $M_{2}$. This means that $C$ is a circuit of exactly one of $M_{3}$ and $M_{4}$ and so, as $C \notin \mathcal{C}_{i, Z}$ for $i \in\{1,2,3,4\}$, the set $C$ is a circuit of exactly one of $N_{3}$ and $N_{4}$. 
Similarly if $C \in \mathcal{C}_{N_{2}}$ and $C \notin \mathcal{C}_{N_{1}}$, the set $C$ is a circuit of exactly one of $N_{3}$ and $N_{4}$.

Finally let $C \notin C_{N_{1}}$ and $C \notin C_{N_{2}}$. This means that $C$ is not a circuit of $M_{1}$ or $M_{2}$ and therefore not a circuit of $M_{3}$ or $M_{4}$, and $C \notin C_{N_{1}}$ and so $C \notin C_{N_{3}}$ and $C \notin C_{N_{4}}$. Therefore $C$ is not a circuit of $N_{3}$ or $N_{4}$.

\section{Proof of Theorem}

We are now almost in a position to prove the main theorem for this section. Before we do this we must prove the following:

Lemma 5.22. Let $M_{1}$ and $M_{2}$ be matroids such that $E\left(M_{1}\right) \cap E\left(M_{2}\right)=\{e\}$. Then $B$ is a basis of $M_{1} \oplus_{2} M_{2}$ if, and only if, $B=\left(B_{1} \cup B_{2}\right)-\{e\}$ where $B_{1}$ and $B_{2}$ are bases on $M_{1}$ and $M_{2}$ respectively such that $e \in B_{1} \triangle B_{2}$.

Proof. Let $B_{1}$ and $B_{2}$ be bases of $M_{1}$ and $M_{2}$ respectively, and suppose that $e \in B_{1} \triangle B_{2}$. Without loss of generality let $e \in B_{2}-B_{1}$. The set of circuits of $M_{1} \oplus M_{2}$ is:

$\mathcal{C}\left(M_{1} \backslash\{e\}\right)+\mathcal{C}\left(M_{2} \backslash\{e\}\right) \cup\left\{(C \cup D)-\{e\}: e \in C \in \mathcal{C}\left(M_{1}\right)\right.$ and $\left.e \in D \in \mathcal{C}\left(M_{2}\right)\right\}$

As neither $B_{1}$ nor $B_{2}$ contains a circuit in $M_{1} \backslash e$ or $M_{2} \backslash e$, and $B_{2} \cup\{e\}$ does not contain a circuit in $M_{1}$, we can see that $\left(B_{1} \cup B_{2}\right)-\{e\}$ does not contain a circuit of $M_{1} \oplus_{2} M_{2}$. This means that $\left(B_{1} \cup B_{2}\right)-\{e\}$ is independent in $M_{1} \oplus_{2} M_{2}$.

To show that $\left(B_{1} \cup B_{2}\right)-\{e\}$ is a basis of $M_{1} \oplus_{2} M_{2}$, we must show that $\left(B_{1} \cup B_{2}\right)-\{e\}$ is maximally independent in $M_{1} \oplus_{2} M_{2}$. Suppose that it is not. Then there is some $f \in E\left(M_{1} \oplus_{2} M_{2}\right)-\left(\left(B_{1} \cup B_{2}\right)-\{e\}\right)$ such that $\left(\left(B_{1} \cup B_{2}\right)-\{e\}\right) \cup\{f\}$ is independent in $M_{1} \oplus_{2} M_{2}$. Note that $f \neq e$ as $e \notin E\left(M_{1} \oplus_{2} M_{2}\right)$. Either $f \in E\left(M_{1}\right)$ or $f \in E\left(M_{2}\right)$. Suppose first that $f \in E\left(M_{1}\right)$. As $B_{1}$ is a basis for $M_{1}$, the set $B_{1} \cup\{f\}$ contains a circuit in $M_{1}$. The circuit of $M_{1}$ contained in $B_{1} \cup\{f\}$ is also a circuit of $M_{1} \backslash e$ and therefore is a circuit of $M_{1} \oplus_{2} M_{2}$. Therefore $\left(\left(B_{1} \cup B_{2}\right)-\{e\}\right) \cup\{f\}$ contains a circuit of $M_{1} \oplus_{2} M_{2}$ when $f \in B_{1}$; a contradiction. Therefore suppose that $f \in E\left(M_{2}\right)$. Then $B_{2} \cup\{f\}$ contains a circuit of $M_{2}$, which we shall call $C_{2}$. If $e \notin C_{2}$, then $C_{2}$ is a circuit of $M_{2} \backslash e$, and therefore a circuit 
of $M_{1} \oplus_{2} M_{2}$, a contradiction and $\left(\left(B_{1} \cup B_{2}\right)\{e\}\right) \cup\{f\}$ is independent in $M_{1} \oplus_{2} M_{2}$. Therefore assume that $e \in C_{2}$. As $B_{1}$ is a basis of $M_{1}$ that does not contain $e$, there is a circuit of $M_{1}$ contained in $B_{1} \cup\{e\}$ that contains $e$. Call this circuit $C_{1}$. As $C_{2}$ is a circuit of $M_{2}$ containing $e$ and $C_{1}$ is a circuit of $M_{1}$ containing $e$, the set $\left(C_{1} \cup C_{2}\right)-\{e\}$ is a circuit of $M_{1} \oplus_{2} M_{2}$; but this is contained in $\left(\left(B_{1} \cup B_{2}\right)-\{e\}\right) \cup\{f\}$ and therefore $\left(\left(B_{1} \cup B_{2}\right)-\{e\}\right) \cup\{f\}$ is not independent. Therefore $\left(B_{1} \cup B_{2}\right)-\{e\}$ is maximally independent in $M_{1} \oplus_{2} M_{2}$ and so is a basis of $M_{1} \oplus_{2} M_{2}$.

Now suppose that we have a basis $B$ of $M_{1} \oplus M_{2}$. Suppose that $B$ is fully contained in $M_{1}$. Then either $|B| \leq r\left(M_{1}\right)$ or $B$ contains a circuit of $M_{1}$. If $B$ contains a circuit of $M_{1}$, then, as $e \notin B$, the set $B$ also contains a circuit of $M_{1} \backslash e$ and therefore a circuit of $M_{1} \oplus_{2} M_{2}$. This contradicts the fact that $B$ is a basis of $M_{1} \oplus M_{2}$, and therefore $B$ cannot contain a circuit of $M_{1}$. Therefore $B$ must be independent in $M_{1}$. If $|B|<r\left(M_{1}\right)$, then $|B|<r\left(M_{1}\right)+r\left(M_{2}\right)-1$, as $r\left(M_{2}\right) \geq 1$, as $e$ is not a loop, and so $|B|<r\left(M_{1} \oplus_{2} M_{2}\right)$, so $B$ is not a basis of $M_{1} \oplus_{2} M_{2}$, a contradiction. Therefore we shall assume that $B$ is a basis for $M_{1}$. Therefore $|B|=r\left(M_{1}\right)$. We know that $r\left(M_{1} \oplus_{2} M_{2}\right)=r\left(M_{1}\right)+r\left(M_{2}\right)-1$, so $B$ can only be a basis for $M_{1} \oplus M_{2}$ if $r\left(M_{2}\right)=1$. If $r\left(M_{2}\right)=1$ then $e$ is a basis for $M_{2}$ as $e$ is not a loop, therefore $B=B_{1} \cup B_{2}-\{e\}$ where $B_{1}=B$, a basis for $M_{1}$ that does not include $e$, and $B_{2}=\{e\}$, a basis for $M_{2}$.

Now suppose that $B$ has a non-empty intersection with both $M_{1}$ and $M_{2}$. Let $B \cap E_{1}=D_{1}$ and $B \cap E_{2}=D_{2}$. Both $D_{1}$ and $D_{2}$ must be independent in $M_{1}$ and $M_{2}$ respectively, as otherwise $B$ would contain a circuit of $M_{1} \oplus_{2} M_{2}$. This means that $D_{1}$ is independent in $M_{1}$ and $D_{2}$ is independent in $M_{2}$. The only way that $D_{1} \cup D_{2}$ could be dependent in $M_{1} \oplus_{2} M_{2}$ would be if both $D_{1} \cup\{e\}$ and $D_{2} \cup\{e\}$ contained circuits of $M_{1}$ and $M_{2}$ respectively, containing $e$. As $B=D_{1} \cup D_{2}$ is a basis for $M_{1} \oplus_{2} M_{2}$, it cannot be that $B$ contains a circuit of $M_{1} \oplus_{2} M$.

Theorem 5.4. Let $M_{1}, M_{2}, M_{3}, M_{4}$ be matroids on a set $E$; then, if $\left(M_{1}, M_{2}\right) \sim\left(M_{3}, M_{4}\right)$ and $\left(M_{1}, M_{2}\right)$ is connected, then $B$ is a base of $M_{1}$ or $M_{2}$ if, and only if, $B$ is a base of $M_{3}$ or $M_{4}$.

Proof. We shall prove that if $B$ is a base of $M_{1}$, then it is a base of $M_{3}$ or $M_{4}$. As the relation $\sim$ is symmetric we could use a similar argument to show 
that if $B$ were a base of $M_{3}$ or $M_{4}$, then $B$ would be a base of $M_{1}$ or $M_{2}$, and the result follows.

The proof is by induction on $|E|$. For the base case assume that $|E| \leq 3$. If $E$ contains an element, $e$, that is a loop of $M_{1}$, then $e$ is also a loop in $M_{3}$ or $M_{4}$ by Lemma 5.13, and so, by Lemma 5.15, $M_{1}=M_{3}$ or $M_{1}=M_{4}$ and the result holds. Similarly if $E$ contains an element that is a loop of $M_{2}$ then $M_{2}=M_{3}$ or $M_{2}=M_{4}$ and so, as $\left(M_{1}, M_{2}\right) \sim\left(M_{3}, M_{4}\right)$, we have $M_{1}=M_{4}$ or $M_{1}=M_{3}$ and the result holds. If $E$ contains an element that is a coloop of $M_{1}$ or of $M_{2}$, then the result holds by duality. Therefore assume that $|E|$ contains no loops or coloops of $M_{1}$ or $M_{2}$. Then the base case follows as there are no such 1-element matroids, one such 2-element matroid, and two such 3-element matroids. The two such 3-element matroids have different rank so the result follows.

Suppose that $\left(M_{1} \backslash\{e\}, M_{2} \backslash\{e\}\right)$ is disconnected for some $e \in E$. Then there is some $A \subseteq E$ such that $\mu_{M_{i} \backslash\{e\}}(A)=0$ for all $i \in\{1,2,3,4\}$. Therefore $\mu_{M_{i}}(A) \leq 1$ for all $i \in\{1,2,3,4\}$. If $\mu_{M_{1}}(A)+\mu_{M_{2}}(A)=1$, then by Lemma 5.20, $M_{1}=M_{3}$ or $M_{1}=M_{4}$ and so the result holds. Therefore we shall assume that $\mu_{M_{i}}(A)=1$ for all $i \in\{1,2,3,4\}$ (note that $\mu_{M_{1}}(A)+\mu_{M_{2}}(A) \neq 0$ as $\left(M_{1}, M_{2}\right) \sim\left(M_{3}, M_{4}\right)$ and $\left(M_{1}, M_{2}\right)$ is connected $)$. This means that by Lemma 5.21, either: $M_{1} \in\left\{M_{3}, M_{4}\right\}$, or there are matroids $N_{i}$ and $N$ for $i \in\{1,2,3,4\}$ such that all the following hold:

i) $\left(N_{1}, N_{2}\right) \sim\left(N_{3}, N_{4}\right)$,

ii) $M_{i}=N_{i} \oplus_{2} N$

iii) $E(N)=A \cup\{e\}$ or $E(N)=(E-A) \cup\{e\}$

If $M_{1} \in\left\{M_{3}, M_{4}\right\}$ then we are done, so assume that such $N_{i}$ and $N$ exist, and without loss of generality let $E(N)=A \cup\{e\}$. By Lemma 5.22 we can say that either:

(a) $B-A$ is a base of $N_{1}$ and $(B \cap A) \cup\{e\}$ is a base of $N$, or

(b) $(B-A) \cup\{e\}$ is a base of $N_{1}$ and $B \cap A$ is a base of $N$.

Note that, as $\left(M_{1}, M_{2}\right)$ is connected, $\left(N_{1}, N_{2}\right)$ is connected. Assume that $(a)$ holds. Then by induction $B-A$ is a base of $N_{3}$ or $N_{4}$, and, as $(B \cap A) \cup\{e\}$ 
is a base of $N$ and $M_{i}=N_{i} \oplus_{2} N$, the set $B$ is a base of $M_{3}$ or $M_{4}$. If $(b)$ holds then a similar argument shows that, again, $B$ is a base of $M_{3}$ or $M_{4}$.

We have now shown that the result holds when $\left(M_{1}, M_{2}\right)$ is connected and $\left(M_{1} \backslash\{e\}, M_{2} \backslash\{e\}\right)$ is disconnected for some $e \in E$. Therefore, for the remainder of the proof, we shall assume that $\left(M_{1} \backslash\{e\}, M_{2} \backslash\{e\}\right)$ is connected for all $e \in E$.

First suppose that $B=E$ and recall that a coloop is not contained in any circuit (see [9]). This means that every $e \in E$ is a coloop of $M_{1}$. Therefore, by duality and Lemma 5.13 , every element of $E$ is a coloop of either $M_{3}$ or $M_{4}$. Let $A_{i}=\left\{e \in E: e\right.$ is a coloop of $\left.M_{i}\right\}$ for $i \in\{3,4\}$. As every element of $E$ is a coloop of either $M_{3}$ or $M_{4}$, the set $A_{3} \cup A_{4}=E$ and, as a coloop is not contained in any circuit, $M_{3}$ and $M_{4}$ do not contain an element intersecting both $A_{3}$ and $A_{4}$. However, as $\left(M_{3}, M_{4}\right)$ is connected, this means that either $A_{3}=\emptyset$ or $A_{4}=\emptyset$, and so either $A_{3}=E$ or $A_{4}=E$, and so either $B$ is a base of $A_{3}$ or $B$ is a base of $A_{4}$.

Suppose that $B \neq E$, and let $e \notin B$. By induction, as $\left(M_{1} \backslash\{e\}, M_{2} \backslash\{e\}\right)$ is connected and $e \notin B$ so $B$ is a base of $M_{1} \backslash\{e\}$, the set $B$ is either a base of $M_{3} \backslash\{e\}$ or $M_{4} \backslash\{e\}$ and so $B$ is a base of either $M_{3}$ or $M_{4}$.

\subsection{Connectivity Polymatroids}

Recall that the connectivity polymatroid generated by a connectivity function $\lambda$ on a set $E$, known as $P_{\lambda}$, is defined by

$$
r_{P_{\lambda}}(X)=\|X\|_{\lambda}+\lambda(X),
$$

for all $X \subseteq E$. The connectivity function of $P_{\lambda}$ is equal to twice $\lambda$.

In [8] a description of when a connectivity polymatroid is the connectivity polymatroid of a matroidal connectivity function is given. We give a slight generalisation of the result and proof here. Note that in this result we use the polymatroid dual, as opposed to the matroid dual.

However, before we do this, we shall need the following two lemmas:

Lemma 5.23. If $\mu$ is the connectivity function of a matroid, then $\mu$ is the 
connectivity function of a coloop-free matroid.

Proof. Let $M_{1}=\left(E, r_{1}\right)$ be a matroid whose connectivity function is $\mu_{M_{1}}$. Let $a$ be a coloop of $M_{1}$ and let $M_{2}=\left(E, r_{2}\right)$ be the matroid defined as follows:

$$
r_{2}(A)= \begin{cases}r_{1}(A) & \text { if } a \notin A \\ r_{1}(A)-1 & \text { if } a \in A .\end{cases}
$$

This is the matroid obtained by replacing the coloop, $a$, of $M_{1}$ by a loop in $M_{2}$. It is fairly easy to check that this is indeed a matroid. This gives

$$
\mu_{M_{2}}(A)= \begin{cases}r_{1}(A)+r_{1}(E-A)-1-\left(r_{1}(E)-1\right) & \text { if } a \notin A \\ r_{1}(A)-1+r_{1}(E-A)-\left(r_{1}(E)-1\right) & \text { if } a \in A\end{cases}
$$

so, in both cases, $\mu_{M_{1}}(A)=\mu_{M_{2}}(A)$. inductively, this means that for any matroidal connectivity function $\mu$, we can find a coloop-free matroid $M$, that is such that $\mu_{M}=\mu$.

The proof of the following theorem is simple.

Lemma 5.24. Let $M=(E, r)$ be a loopless matroid with connectivity function $\mu_{M}$. Then, for all $X \subseteq E$ we have $\mu_{M}(X)=r(X)+r_{M^{*}}(X)-\|X\|_{r}$, where $r_{M^{*}}$ is the polymatroid dual of $M$.

Theorem 5.25. Let $\lambda$ be a unitary connectivity function on a set $E$, and let $P_{\lambda}$ be the connectivity polymatroid generated by $\lambda$. Then $\lambda$ is a matroidal connectivity function if, and only if, there is some matroid, $M$, on $E$ with rank function $r$ such that:

$$
r_{P_{\lambda}}(X)=r_{M}(X)+r_{M^{*}}(X)
$$

where $r^{*}$ is the rank function of the polymatroid dual of $M$, and $X \subseteq E$.

Proof. Suppose that $\lambda(X)$ is matroidal:

$\lambda(X)$ is matroidal

$\Longrightarrow \lambda(X)=r_{M}(X)+r_{M^{*}}(X)-\|X\|_{r_{M}}$ where $M$ is a coloop-free matroid

$\Longrightarrow \lambda(X)+\|X\|_{r_{M}}=r_{M}(X)+r_{M^{*}}(X)$

$\Longrightarrow \lambda(X)+\|X\|_{\lambda}=r_{M}(X)+r_{M^{*}}(X)$ as $M$ is coloop-free. 
Suppose that $r_{P_{\lambda}}(X)=r_{M}(X)+r_{M^{*}}(X)$.

$$
\begin{aligned}
& r_{P_{\lambda}}(X)=r_{M}(X)+r_{M^{*}}(X) \\
& \Longrightarrow \lambda(X)+\|X\|_{\lambda}=r_{M}(X)+r_{M^{*}}(X) \\
& \Longrightarrow \lambda(X)+\|X\|_{\lambda}=\mu_{M}(X)+\|X\|_{r_{M}}
\end{aligned}
$$

where (2) follows from (1) by definition of $r_{P_{\lambda}}(X)$, and (3) follows from (2) as $\mu_{M}(X)=r_{M}(X)+r_{M^{*}}(X)-\|X\|_{r_{M}}$.

When $X=\{a\}$ for any $A \subseteq E$, we have $2 \lambda(\{a\})=\mu_{M}(\{a\})+r_{M}(\{a\})$. Therefore, as $\lambda$ and $\mu_{M}$ are both unitary and $r_{M}(\{a\}) \leq 1$, we have $r_{M}(\{a\})=\mu_{M}(\{a\})=\lambda(\{a\})$, so $\|X\|_{\lambda}=\|X\|_{r_{M}}$. Therefore $\lambda(X)=$ $\mu_{M}(X)$ for any $X \subseteq E$, and so $\lambda$ is matroidal.

\subsection{Spikes}

Definition 5.26. Let $n$ be an integer greater than 2. A matroid $M$ with the following properties is a rank-n spike:

1. $E(M)$ is the union of $n$ lines $L_{1}, \ldots, L_{n}$ where each line contains two points. $L_{1}, \ldots, L_{n}$ will be known as the legs of the spike (and each $L_{a_{i}}$ will be a leg).

2. For all $k$ in $\{1, \ldots, n-1\}$, the union of any $k$ legs has rank $k+1$.

3. $r\left(L_{1} \cup L_{2} \cup \ldots \cup L_{n}\right)=n$

The next result is taken from [9]:

Theorem 5.27. A pair $M=(E, \mathcal{C})$, where $M$ is a matroid with collection of circuits $\mathcal{C}$, is a rank-n spike, if, and only if, $E$ can be partitioned into pairs $\left\{x_{i}, y_{i}\right\}$ such that $\mathcal{C}$ is equal to $\mathcal{C}_{1} \cup \mathcal{C}_{2} \cup \mathcal{C}_{3}$ where $\mathcal{C}_{1}=\left\{\left\{x_{i}, y_{i}, x_{j}, y_{j}\right\}: 1 \leq\right.$ $i<j \leq r\}, \mathcal{C}_{2}$ is a, possibly empty, subset of $\left\{\left\{z_{1}, \ldots, z_{n}\right\}: z_{i} \in\left\{x_{i}, y_{i}\right\}\right\}$ such that no two members of $\mathcal{C}_{2}$ differ in exactly one element, and $\mathcal{C}_{3}$ is the collection of all $n+1$ element subsets of $E(M)$ that contain no member of $\mathcal{C}_{1} \cup \mathcal{C}_{2}$ 
The circuits in $\mathcal{C}_{2}$ are known as the transversal circuits of the spike. When talking about spikes we shall use $z_{i}$ to describe a single element of $\left\{x_{i}, y_{i}\right\}$, and we shall refer to $\left\{z_{1}, \ldots, z_{n}\right\}$ as a transversal of a rank- $n$ spike.

Throughout this section we shall use the following results:

Lemma 5.28. Let $S$ be a spike with legs $L_{1}, \ldots, L_{n}$ and connectivity function $\mu$. Then

i) $\mu\left(\left\{z_{a_{1}}, \ldots, z_{a_{k}}\right\}\right)=k$.

ii) $\mu\left(L_{a_{1}} \cup \cdots \cup L_{a_{k}}\right)=2$.

iii) $\mu\left(L_{a_{1}} \cup \cdots \cup L_{a_{k}} \cup\left\{z_{a_{k+1}}, \ldots, z_{a_{m}}\right\}\right)=m-k+2$.

where $k \leq m<n$ and $a_{i} \in\{1, \ldots, n\}$.

Proof.

i) $r\left(\left\{z_{a_{1}}, \ldots, z_{a_{k}}\right\}\right)=k$ and $r\left(E-\left\{z_{a_{1}}, \ldots, z_{a_{k}}\right\}\right)=r(E)$. Therefore $\mu\left(\left\{z_{a_{1}}, \ldots, z_{a_{k}}\right\}\right)=r\left(\left\{z_{a_{1}}, \ldots, z_{a_{k}}\right\}\right)=k$.

ii) $r\left(L_{a_{1}} \cup \cdots \cup L_{a_{k}}\right)=k+1$ and $r\left(E-\left(L_{a_{1}} \cup \cdots \cup L_{a_{k}}\right)\right)=n-k+1$. Therefore $\mu\left(L_{a_{1}} \cup \cdots \cup L_{a_{k}}\right)=r\left(L_{a_{1}} \cup \cdots \cup L_{a_{k}}\right)+r\left(E-\left(L_{a_{1}} \cup \cdots \cup L_{a_{k}}\right)\right)-r(E)=2$.

iii) It is easy to see that $r\left(L_{a_{1}} \cup \cdots \cup L_{a_{k}} \cup\left\{z_{a_{k+1}}, \ldots, z_{a_{m}}\right\}\right)=m+1$ and $r\left(E-\left(L_{a_{1}} \cup \cdots \cup L_{a_{k}} \cup\left\{z_{a_{k+1}}, \ldots, z_{a_{m}}\right\}\right)\right)=n-k+1$. Therefore we must have $\mu\left(L_{a_{1}} \cup \cdots \cup L_{a_{k}} \cup\left\{z_{a_{k+1}}, \ldots, z_{a_{m}}\right\}\right)=m+1+n-k+1-n=m-k+2$.

Lemma 5.29. Let $\mu$ be the connectivity function of a spike with legs $L_{1}, \ldots, L_{n}$ where $n \geq 3$, and let $\lambda$ be a connectivity function that agrees with $\mu$ everywhere except possibly on transversal sets. If there exists a matroid, $M$, such that $\mu_{M}=\lambda$, then $M$ is a spike.

Proof. Let $M=(E, r)$ be a matroid such that $\mu_{M}=\lambda$. We do not yet know that we have a spike, but we shall refer to pairs $\left\{x_{i}, y_{i}\right\}$ as legs. We shall also use $a_{i}$ to denote some integer in $\{1, \ldots, n\}$. We need to show that the union of any $k$ legs has rank $k+1$ for all $k \in\{1, \ldots, n-1\}$, and the rank of the union 
of $n$ legs is equal to $n$. First we shall look at the rank of $k$ legs for $k<n$, and without loss of generality we shall let those legs be $L_{a_{1}} \cup \cdots \cup L_{a_{k}}$. As $\mu$ is the connectivity function of a spike $\mu\left(L_{a_{1}} \cup \cdots \cup L_{a_{k}}\right)=2$, and so $\lambda\left(L_{a_{1}} \cup \cdots \cup L_{a_{k}}\right)=2$. Therefore, $r\left(L_{a_{1}} \cup \cdots \cup L_{a_{k}}\right)+r^{*}\left(L_{a_{1}} \cup \cdots \cup\right.$ $\left.L_{a_{k}}\right)=2 k+2$. We also know that $\mu\left(L_{a_{1}} \cup\left\{z_{2}, \ldots, z_{a_{k}}\right\}\right)=k+1$ and so $\lambda\left(L_{a_{1}} \cup\left\{z_{2}, \ldots, z_{a_{k}}\right\}\right)=k+1$, and therefore this matroid would also have to have $r\left(L_{a_{1}} \cup\left\{z_{2}, \ldots, z_{a_{k}}\right\}\right)+r^{*}\left(L_{a_{1}} \cup\left\{z_{2}, \ldots, z_{a_{k}}\right\}\right)-(k+1)=k+1$. Therefore $r\left(L_{a_{1}} \cup\left\{z_{2}, \ldots, z_{a_{k}}\right\}\right)=k+1=r^{*}\left(L_{a_{1}} \cup\left\{z_{2}, \ldots, z_{a_{k}}\right\}\right)$. Therefore $r\left(L_{a_{1}} \cup \cdots \cup L_{a_{k}}\right) \geq k+1$ and $r^{*}\left(L_{a_{1}} \cup \cdots \cup L_{a_{k}}\right) \geq k+1$ and as $r\left(L_{a_{1}} \cup \cdots \cup\right.$ $\left.L_{a_{k}}\right)+r^{*}\left(L_{a_{1}} \cup \cdots \cup L_{a_{k}}\right)=2 k+2$, it must be that $r\left(L_{a_{1}} \cup \cdots \cup L_{a_{k}}\right)=k+1$. Now consider $r\left(L_{a_{1}} \cup \cdots \cup L_{a_{n}}\right)$. We know that $r\left(L_{a_{1}} \cup \cdots \cup L_{a_{n}}\right)+$ $r^{*}\left(L_{a_{1}}, \ldots, L_{a_{n}}\right)=2 n$ and, as $r\left(L_{a_{1}} \cup \cdots \cup L_{a_{n-1}}\right)=n=r^{*}\left(L_{a_{1}} \cup \cdots \cup L_{a_{n-1}}\right)$ we have $r\left(L_{a_{1}} \cup \cdots \cup L_{a_{n}}\right) \geq n$ and $r^{*}\left(L_{a_{1}} \cup \cdots \cup L_{a_{n}}\right) \geq n$. Therefore $r\left(L_{a_{1}} \cup \cdots \cup L_{a_{n}}\right)=n$.

We have now shown that $M$ satisfies the definition of a spike.

For the remainder of this section we shall let $a_{i} \in\{1, \ldots, 2 n\}$.

Lemma 5.30. Let $E=\left\{x_{1}, y_{1}, \ldots, x_{2 n}, y_{2 n}\right\}$ be a set and $\mathcal{C}$ be a collection of subsets of $E$ that contains

i) all sets of the form $\left\{x_{i}, y_{i}, x_{j}, y_{j}\right\}$ for $i \neq j$ and $i, j \in\{1, \ldots, 2 n\}$,

ii) all sets of the form $\left\{z_{1}, \ldots, z_{2 n}\right\}$ such that $\left|\left\{z_{1}, \ldots, z_{2 n}\right\} \cap\left\{y_{1}, \ldots, y_{2 n}\right\}\right|$ is even and is strictly less than $n$.

iii) all sets of the form $\left\{z_{1}, \ldots, z_{2 n}\right\}$, such that $\left|\left\{z_{1}, \ldots, z_{2 n}\right\} \cap\left\{y_{1}, \ldots, y_{2 n}\right\}\right|$ is odd and is strictly greater than $n$ and

iv) all sets of $2 n+1$ elements that do not contain any of the subsets described above.

Then $(E, \mathcal{C})$ is a spike with $\mathcal{C}$ as its collection of circuits.

Proof. By Theorem $5.27(E, \mathcal{C})$ will be a spike if, and only if, no pair of elements of the form $\left\{z_{1}, \ldots, z_{2 n}\right\}$ that are contained in $\mathcal{C}$, differ in exactly one element. It is clear that we have this property. 
For the remainder of this section we shall call this spike $S$ and let its connectivity function be $\mu$.

Lemma 5.31. Let $E=\left\{x_{1}, y_{1}, \ldots, x_{2 n}, y_{2 n}\right\}$ be a set and $\mathcal{C}$ be a collection of subsets of $E$ that contains

i) all sets of the form $\left\{x_{i}, y_{i}, x_{j}, y_{j}\right\}$ for $i \neq j$ and $i, j \in\{1, \ldots, 2 n\}$,

ii) all sets of the form $\left\{z_{1}, \ldots, z_{2 n}\right\}$ such that $\left|\left\{z_{1}, \ldots, z_{2 n}\right\} \cap\left\{y_{1}, \ldots, y_{2 n}\right\}\right|$ is even and is strictly less than $n$.

iii) all sets of the form $\left\{z_{1}, \ldots, z_{2 n}\right\}$ such that $\left|\left\{z_{1}, \ldots, z_{2 n}\right\} \cap\left\{y_{1}, \ldots, y_{2 n}\right\}\right|$ is odd and is strictly greater than $n$. ,

iv) the set $\left\{y_{a_{1}}, \ldots, y_{a_{n}}, x_{a_{n+1}}, \ldots, x_{a_{2 n}}\right\}$, and

v) all sets of $2 n+1$ elements that do not contain any of the subsets described above.

Then $\mathcal{C}$ is not the collection of circuits of a spike.

Proof. By Theorem 5.27, $(E, \mathcal{C})$ will be a spike if, and only if, no pair of elements of the form $\left\{z_{1}, \ldots, z_{2 n}\right\}$ that are contained in $\mathcal{C}$ differ in exactly one element. If $n$ is odd, then the set $\left\{y_{1}, \ldots, y_{n-1}, x_{n}, \ldots, x_{2 n}\right\}$ is in $\mathcal{C}$ and differs from $\left\{y_{1}, \ldots, y_{n}, x_{n+1}, \ldots, x_{2 n}\right\}$ (which is also a member of $\mathcal{C}$ ), in exactly one element. If $n$ is even, then the set $\left\{y_{1}, \ldots, y_{n+1}, x_{n+2}, \ldots, x_{2 n}\right\}$ is in $\mathcal{C}$ and differs from $\left\{y_{1}, \ldots, y_{n}, x_{n+1}, \ldots, x_{2 n}\right\}$, in exactly one element. Therefore, in neither case can $\mathcal{C}$ be the collection of circuits of a spike.

Lemma 5.32. Let $\mu$ be the connectivity function of the rank-2n spike whose collection of circuits is:

i) all sets of the form $\left\{x_{i}, y_{i}, x_{j}, y_{j}\right\}$ for $i \neq j$ and $i, j \in\{1, \ldots, 2 n\}$,

ii) all sets of the form $\left\{z_{1}, \ldots, z_{2 n}\right\}$ such that $\left|\left\{z_{1}, \ldots, z_{2 n}\right\} \cap\left\{y_{1}, \ldots, y_{2 n}\right\}\right|$ is even and is strictly less than $n$.

iii) all sets of the form $\left\{z_{1}, \ldots, z_{2 n}\right\}$, such that $\left|\left\{z_{1}, \ldots, z_{2 n}\right\} \cap\left\{y_{1}, \ldots, y_{2 n}\right\}\right|$ is odd and is strictly greater than $n$ and 
iv) all sets of $2 n+1$ elements that do not contain any of the subsets described above.

Let $\lambda$ be a set-valued function on the same set as $\mu$ that agrees with $\mu$ in all sets except on the two sets $\left\{y_{a_{1}}, \ldots, y_{a_{n}}, x_{a_{n+1}}, \ldots, x_{a_{2 n}}\right\}$ and $\left\{x_{a_{1}}, \ldots, x_{a_{n}}, y_{a_{n+1}}, \ldots, y_{a_{2 n}}\right\}$, which have $\lambda\left(\left\{x_{a_{1}}, \ldots, x_{a_{n}}, y_{a_{n+1}}, \ldots, y_{a_{2 n}}\right\}\right)=$ $n-1=\lambda\left(\left\{y_{a_{1}}, \ldots, y_{a_{n}}, x_{a_{n+1}}, \ldots, x_{a_{2 n}}\right\}\right)$. Then $\lambda$ is a connectivity function.

Proof. We need to show that $\lambda$ is submodular, that is $\lambda(A)+\lambda(B) \geq$ $\lambda(A \cup B)+\lambda(A \cap B)$ for all $A, B \subseteq E$. If neither $A$ nor $B$ is equal to $\left(\left\{x_{a_{1}}, \ldots, x_{a_{n}}, y_{a_{n+1}}, \ldots, y_{a_{2 n}}\right\}\right.$ or $\left(\left\{y_{a_{1}}, \ldots, y_{a_{n}}, x_{a_{n+1}}, \ldots, x_{a_{2 n}}\right\}\right.$, then this holds by the submodularity of $\mu$ and by noting that for any $X \subseteq E$, we have $\lambda(X) \leq \mu(X)$.

If either $A \subseteq B$ or $B \subseteq A$ then the inequality is trivial, so we shall suppose without loss of generality, that $A$ is equal to $\left\{y_{1}, \ldots, y_{n}, x_{n+1}, \ldots, x_{2 n}\right\}$, and that $A$ is not a subset of $B$ and $B$ dis not a subset of $A$.

Suppose that $B=\left\{z_{1}, \ldots, z_{2 n}\right\}$. Then $\lambda(B), \lambda(A) \geq 2 n-1$. We have $\lambda(A \cup B)=\mu(A \cup B)=2 n-k$ where $k$ is the number of legs fully contained in $A \cup B$, and $\lambda(A \cap B)=\mu(A \cap B)=2 n-k$ where $k$ is as above. As $A$ and $B$ are both transversal sets and are not equal, we have $k \geq 1$. Therefore:

$$
\begin{aligned}
\lambda(A)+\lambda(B) & \geq(2 n-1)+(2 n-1) \\
& \geq(2 n-k)+(2 n-k) \\
& =\lambda(A \cup B)+\lambda(A \cap B) .
\end{aligned}
$$

Suppose that $B$ is not a transversal but contains elements from all legs. Then $\lambda(B)=2 n-k$ where $k$ is the number of legs that contain two elements of $B$. We also have $\lambda(A \cup B)=2 n-m$ where $m$ is the number of legs of $A \cup B$ that contain two elements of $A \cup B$, and $\lambda(A \cap B)=(2 n-m)+k$. Note that, as we are assuming that $A \nsubseteq B$ we have $m>k$, so:

$$
\begin{aligned}
\lambda(A)+\lambda(B) & =(2 n-1)+(2 n-k) \\
& \geq(2 n-m+k)+2 n-m \\
& =\lambda(A \cup B)+\lambda(A \cap B) .
\end{aligned}
$$


Suppose that $B$ is of the form $\left\{z_{a_{1}}, \ldots, z_{a_{m}}\right\}$ where $m<n$. Then $\lambda(B)=m$. We also have $\lambda(A \cup B)=2 n-k$ where $k$ is the number of legs that contain two elements of $A \cup B$ (note that as $B \nsubseteq A$ we have $k \geq 1$ ) and $\lambda(A \cap B)<m$ as $B \nsubseteq A$. Therefore:

$$
\begin{aligned}
\lambda(A)+\lambda(B) & =2 n-1+m \\
& \geq 2 n-k+m \\
& \geq \lambda(A \cup B)+\lambda(A \cap B) .
\end{aligned}
$$

Finally suppose that $B$ is not a subset of a transversal and does not contain an element of every leg, so $B$ is of the form $\left\{L_{a_{1}} \cup \cdots \cup L_{a_{k}} \cup\left\{z_{a_{k+1}}, \ldots, z_{m}\right\}\right\}$. This means that $\lambda\left(\left\{L_{a_{1}} \cup \cdots \cup L_{a_{k}} \cup\left\{z_{a_{k+1}}, \ldots, z_{m}\right\}\right\}\right)=m-k+2$. Consider $A \cup B$ and $A \cap B$. We have $\lambda(A \cup B)=2 n-l$ where $l$ is the number of legs fully contained in $A \cup B$, and $\lambda(A \cap B) \leq m$ as $A \cap B$ is a strict subset of $A$ and $A \cap B$ is a subset of a transversal. Note that $k \leq l$. Therefore:

$$
\begin{aligned}
\lambda(A)+\lambda(B) & =2 n-1+m-k+2 \\
& =2 n+m-k+1 \\
& >2 n-l+m \\
& \geq \lambda(A \cup B)+\lambda(A \cap B)
\end{aligned}
$$

This means that in every case $\lambda(A)+\lambda(B) \geq \lambda(A \cup B)+\lambda(A \cap B)$, and so $\lambda$ is submodular.

The result we proved is actually slightly stronger than the one stated in the lemma. We proved that, given a spike with connectivity function $\mu$ that has connectivity at least $n-1$ on all transversals, for any set $X=\left\{z_{1}, \ldots, z_{2 n}\right\}$, if we put $\lambda(Y)=\mu(Y)$ for all $Y \neq X,(E-X)$ and $\lambda(X)=2 n-1=\lambda(E-X)$, then $\lambda$ is a connectivity function. However, the result stated in the lemma is sufficient for our purposes.

Lemma 5.33. The connectivity function, $\lambda$, described in 5.32 cannot be the connectivity function of any matroid.

Proof. For the purposes of contradiction let $M$ be a matroid with connectivity function $\lambda$. As $\lambda=\mu$ on all non-transversal subsets of $E$, by Lemma 5.29 . 
$M$ must be a spike. For any $k \neq n$ we have $\lambda\left(\left\{x_{a_{1}}, \ldots, x_{a_{k}}, y_{a_{k+1}}, \ldots, y_{a_{2 n}}\right\}\right)=$ $2 n-1$ and so, for any set of the form $\left\{x_{a_{1}}, \ldots, x_{a_{k}}, y_{a_{k+1}}, \ldots, y_{a_{2 n}}\right\}$ where $k \neq n$, exactly one of it and its complement is a circuit of $M$. This means that exactly one of $\left\{x_{1}, \ldots x_{2 n}\right\}$ and $\left\{y_{1}, \ldots, y_{2 n}\right\}$ is a circuit of $M$. Without loss of generality let $\left\{x_{1}, \ldots, x_{2 n}\right\}$ be a circuit of $M$. This forces all sets of the form $\left\{z_{1}, \ldots, z_{2 n}\right\}$ such that $\left|\left\{z_{1}, \ldots, z_{2 n}\right\} \cap\left\{y_{1}, \ldots, y_{2 n}\right\}\right|$ is strictly less than $n$ and is even, and all sets of the form $\left\{z_{1}, \ldots, z_{n}\right\}$ such that $\left|\left\{z_{1}, \ldots, z_{2 n}\right\} \cap\left\{y_{1}, \ldots, y_{2 n}\right\}\right|$ is strictly greater than $n$ and is odd, to be circuits of $M$. As $\lambda\left(\left\{x_{a_{1}}, \ldots, x_{a_{n}}, y_{a_{n+1}}, \ldots, y_{a_{2 n}}\right\}\right)=2 n-1$ either it or its complement must be a circuit of $M$, which contradicts Lemma 5.31, which states that no such spike can exist.

Theorem 5.34. Let $p$ be any polynomial. Let $\lambda$ be a connectivity function of set $E$ such that $|E|=n$. We cannot recognise in $p(n)$ of calls to the connectivity function oracle, whether a connectivity function is matroidal.

Proof. Consider the connectivity function $\mu$, which is the connectivity function of $S$, the spike whose collection of circuits is $\mathcal{C}$, where $\mathcal{C}$ is described as follows:

i) all sets of the form $\left\{x_{i}, y_{i}, x_{j}, y_{j}\right\}$ for $i \neq j$ and $i, j \in\{1, \ldots, 2 n\}$,

ii) all sets of the form $\left\{z_{1}, \ldots, z_{2 n}\right\}$ such that $\left|\left\{z_{1}, \ldots, z_{2 n}\right\} \cap\left\{y_{1}, \ldots, y_{2 n}\right\}\right|$ is even and is strictly less than $n$.

iii) all sets of the form $\left\{z_{1}, \ldots, z_{2 n}\right\}$, such that $\left|\left\{z_{1}, \ldots, z_{2 n}\right\} \cap\left\{y_{1}, \ldots, y_{2 n}\right\}\right|$ is odd and is strictly greater than $n$ and

iv) all sets of $2 n+1$ elements that do not contain any of the subsets described above.

As $\mu$ is the connectivity function of $S$, we know that $\mu$ is matroidal. Now consider the connectivity function $\lambda$ which is equal to $\mu$ except on the two sets $\left\{x_{a_{1}}, \ldots, x_{a_{n}}, y_{a_{n+1}}, \ldots, y_{a_{2 n}}\right\}$ and $\left\{y_{a_{1}}, \ldots, y_{a_{n}}, x_{a_{n+1}}, \ldots, x_{a_{2 n}}\right\}$ which have $\lambda\left(\left\{x_{a_{1}}, \ldots, x_{a_{n}}, y_{a_{n+1}}, \ldots, y_{a_{2 n}}\right\}\right)=2 n-1=\lambda\left(\left\{y_{a_{1}}, \ldots, y_{a_{n}}, x_{a_{n+1}}, \ldots, x_{a_{2 n}}\right\}\right)$. From Lemma 5.33 we know that $\lambda$ is not the connectivity function of any matroid. However, to distinguish $\lambda$ and $\mu$ we must check, in the worst case, the connectivity of half (due to symmetry) the sets of the form 
$\left\{x_{a_{1}}, \ldots, x_{a_{n}}, y_{a_{n+1}}, \ldots, y_{2 n}\right\}$, that is the connectivity of $\frac{1}{2}\left(\begin{array}{c}2 n \\ n\end{array}\right)$ sets, a nonpolynomial number.

As, by Lemma 5.29, the connectivity function of a spike cannot be the connectivity function of any non-spike matroid, we cannot tell, in a polynomial number of evaluations of the connectivity function, when a matroidal connectivity function is the connectivity function of a binary matroid. The proof of this is very similar to the proof that we cannot, in a polynomial number of rank evaluations, determine if a matroid is binary. This proof can be found in [11. Given that we cannot recognise, in a polynomial number of rank evaluations, when a matroid is binary, it is not surprising that we cannot recognise when a connectivity function is binary in a polynomial number of evaluations of the connectivity function, but it is worth noting as it shows that, in this case anyway, connectivity functions behave as we expect them to.

\subsection{Self-Dual Matroids}

Recall that the dual of the matroid $M=(E, r)$ is the matroid $M^{*}=\left(E, r^{*}\right)$ where $r^{*}$ is defined as follows:

$$
r^{*}(X)=|X|+r(E-X)-r(M)
$$

for all $X \subseteq E$.

Recall that the connectivity function of a matroid, $M$, is $\mu_{M}(X)=r(X)+$ $r(E-X)-r(M)$, or, equivalently, $\mu_{M}(X)=r(X)+r^{*}(X)-|X|$. An identically self-dual matroid, $M$, is one in which $r(X)=r^{*}(X)$ for all $X \subseteq E$, so, for an identically self-dual matroid, $\mu_{M}(X)=2 r(X)-|X|$.

For the remainder of this section we shall let $\mu$ be the connectivity function of an identically self-dual matroid $M$, and let $\lambda$ be a connectivity function which agrees with $\mu$ on all the following sets

i) all circuits (and therefore cocircuits),

ii) all sets $X$ such that there is some $e \in E$ such that $X \cup\{e\}$ is a circuit, 
iii) singletons.

Note that no self-dual matroid contains loops or coloops, as a coloop is contained in every basis so must have rank equal to one, and a loop must have rank 0 .

\section{Lemma 5.35.}

i) If $C$ is a circuit of $M$ then $\mu(C)=r(C)-1$

ii) If $X$ is an independent set then $\mu(X)=r(X)$

Proof.

i) If $C$ is a circuit of $M$ then $r(C)=|C|-1$ so

$$
\mu(C)=2|C|-2-|C|=|C|-2=r(C)-1
$$

ii) is clear.

Lemma 5.36. Suppose that $X$ is independent, then $\mu(X)=\lambda(X)$.

Proof. We first prove that $\mu(X) \geq \lambda(X)$. We do this by noting that $X$ is independent, and therefore $\mu(X)=r(X)=|X|$. As $\lambda$ is unitary, $\lambda(X) \leq$ $|X|=\mu(X)$.

We now prove that $\mu(X) \leq \lambda(X)$. If there is some circuit, $C$, of $M$ such that $X \subseteq C$ then we proceed by induction on $|C-X|$, otherwise the induction is on $|E-X|$. By our conditions on $\lambda$ the result holds when $|C-X| \leq 1$ and when $|E-X| \leq 1$. For the purposes of induction assume that the result holds for any $X^{\prime}$ such that $\left|C-X^{\prime}\right|<|C-X|$, or, if there is not $C$ such that $C \supseteq X$, then assume that the results holds for all $X^{\prime}$ such that $\left|E-X^{\prime}\right|<|E-X|$. Assume that $|C-X| \geq 2$ (or $|E-X| \geq 2$ ), then $X \cup\{e\}$ is independent for $e \in C-X$ (or in $E-X$ if no $C \supseteq X$ exists) and 
so $\mu(X)+\mu(\{e\})=\mu(X \cup\{e\})$. Therefore:

$$
\begin{aligned}
\mu(X)+\mu(\{e\}) & =\mu(X \cup\{e\}) \\
& \leq \lambda(X \cup\{e\}) \\
& \leq \lambda(X)+\lambda(\{e\}) \\
& =\lambda(X)+\mu(\{e\}),
\end{aligned}
$$

where (2) follows from (1) by the induction hypothesis, (3) follows from (2) by submodularity, and (4) follows from (3) by the conditions on $\lambda$. Therefore $\mu(X) \leq \lambda(X)$.

Lemma 5.37. Suppose that $X$ is dependent, then $\mu(X)=\lambda(X)$

Proof. We first show that $\mu(X) \leq \lambda(X)$. We do this by induction on $|E-X|$. If $|E-X| \leq 1$ then the result holds by the definition of $\lambda$. Suppose first that $r(X \cup\{e\})=r(X)$ for some $e \in E-X$. Then $e \in \operatorname{cl}(X)$ and so, as $X$ contains a circuit, $e$ is contained in a circuit $C_{e} \subsetneq X \cup\{e\}$, (see [9] Proposition 1.4.11). We next need the following equalities:

$$
\begin{aligned}
\mu(X \cup\{e\}) & =2 r(X \cup\{e\})-|X \cup\{e\}| \\
& =2 r(X)-|X|-1 \\
& =\mu(X)-1,
\end{aligned}
$$

and

$$
\begin{aligned}
\mu\left(C_{e}-\{e\}\right) & =2 r\left(C_{e}-\{e\}\right)-\left|C_{e}-\{e\}\right| \\
& =2 r\left(C_{e}\right)-\left(\left|C_{e}\right|-1\right) \\
& =2 r\left(C_{e}\right)-r\left(C_{e}\right) \\
& =r\left(C_{e}\right) \\
& =\mu\left(C_{e}\right)+1 .
\end{aligned}
$$


Therefore $\mu(X)+\mu\left(C_{e}\right)=\mu(X \cup\{e\})+\mu\left(C_{e}-\{e\}\right)$ and so:

$$
\begin{aligned}
\mu(X)+\mu\left(C_{e}\right) & =\mu(X \cup\{e\})+\mu\left(C_{e}-\{e\}\right) \\
& \leq \lambda(X \cup\{e\})+\lambda\left(C_{e}-\{e\}\right) \\
& \leq \lambda(X)+\lambda\left(C_{e}\right) \\
& =\lambda(X)+\mu\left(C_{e}\right),
\end{aligned}
$$

where (2) follows from (1) by the induction hypothesis and conditions on $\lambda$, (3) follows from (2) by submodularity, and (4) follows from (3) by the conditions on $\lambda$.

Suppose now that $r(X \cup\{e\})=r(X)+1$. Note that $r(\{e\})=1$ and so $\mu(\{e\})=1$ as $M$ is self dual. Therefore:

$$
\begin{aligned}
\mu(X \cup\{e\}) & =2 r(X \cup\{e\})-|X \cup\{e\}| \\
& =2(r(X)+1)-|X|-1 \\
& =2 r(X)-|X|+1 \\
& =\mu(X)+r(\{e\}) \\
& =\mu(X)+\mu(\{e\})
\end{aligned}
$$

Therefore:

$$
\begin{aligned}
\mu(X)+\mu(\{e\}) & =\mu(X \cup\{e\}) \\
& \leq \lambda(X \cup\{e\}) \\
& \leq \lambda(X)+\lambda(\{e\}) \\
& =\lambda(X)+\mu(\{e\}) .
\end{aligned}
$$

where (2) follows from (1) by the induction hypothesis, (3) follows from (2) by submodularity, and (4) follows from (3) by conditions on $\lambda$.

We now show that $\mu(X) \geq \lambda(X)$.

As $X$ is dependent there is some circuit $C \subseteq X$; we proceed by induction on $|X|$, keeping $C$ fixed. If $X=C$ then the result holds by conditions on $\lambda$.

If $r(X)=r(X-\{e\})$, then there is some circuit $C_{e} \subseteq X$ such that $e \in C_{e}$ 
(again, see [9], Proposition 1.4.11). Therefore:

$$
\begin{aligned}
\mu(X)+\mu\left(C_{e}-\{e\}\right) & =\mu(X-\{e\})+\mu\left(C_{e}\right) \\
& \geq \lambda(X-\{e\})+\lambda\left(C_{e}\right) \\
& \geq \lambda(X)+\lambda\left(C_{e}-\{e\}\right) \\
& =\lambda(X)+\mu\left(C_{e}-\{e\}\right),
\end{aligned}
$$

where (2) follows from (1) by the induction hypothesis and conditions of $\lambda$, (3) follows from (2) by submodularity, and (4) follows from (3) by the conditions on $\lambda$.

If $r(X) \neq r(X-\{e\})$, then $r(X)=r(X-\{e\})+1$, then

$$
\begin{aligned}
\mu(X-\{e\}) & =2 r(X-\{e\})-|X-\{e\}| \\
& =2(r(X)-1)-|X|+1 \\
& =2 r(X)-|X|-1 \\
& =\mu(X)-1
\end{aligned}
$$

and $\mu(\{e\})=1$, as $e$ is not a loop or a coloop. Therefore:

$$
\begin{aligned}
\mu(X) & =\mu(X-\{e\})+\mu(\{e\}) \\
& \geq \lambda(X-\{e\})+\lambda(\{e\}) \\
& \geq \lambda(X),
\end{aligned}
$$

where (2) follows from (1) by the induction hypothesis, and (3) follows from (2) by submodularity.

Theorem 5.38. Let $\lambda$ be a connectivity function on a set $E$ and let $\mathcal{C}$ be the set of all $C \subseteq E$ such that $\lambda(C)<|C|$ and $C$ is minimal with respect to this property. Then $\lambda$ is the connectivity function of an identically self-dual matroid if, and only if, $\lambda$ is unitary and both the following properties hold:

1. $\lambda(C)=|C|-2$ for all $C \in \mathcal{C}$

2. $\mathcal{C}$ is the collection of circuits of an identically self-dual matroid.

Proof. If $\lambda$ is the connectivity function of an identically self-dual matroid, $M$, then the circuits of $M$ are the sets $C$ that have $\lambda(C)=|C|-2$ and are minimal 
with respect to this property. Every subset of a circuit is independent so has connectivity equal to its size. Therefore the circuits of $C$ are the sets such that $\lambda(C)<|C|$ and $C$ is minimal with respect to this property. In other words $M$ has collection of circuits $\mathcal{C}$, where $\mathcal{C}$ is as described in the statement of the theorem. It is then easy to see that $i$ ) and $i i$ ) hold.

Conversely if $\mathcal{C}$ is the collection of circuits of an identically self-dual matroid, then let $M$ be the matroid with collection of circuits $\mathcal{C}$. The connectivity function, $\mu_{M}$, of $M$ will have $\mu_{M}(C)=|C|-2=\lambda(C)$ and for any $X \subsetneq C$, by the minimality of $C$, we will have $\mu(X)=|X|=\lambda(X)$. In particular $\lambda(X)=\mu_{M}(X)$ when $X$ is a circuit, a singleton, or when there is some $e$ such that $X \cup\{e\}$ is a circuit of $M$. Therefore $\mu_{M}=\lambda$ and so $\lambda$ is the connectivity function of an identically self-dual matroid. 


\section{Chapter 6}

\section{Branch-Width}

Definition 6.1. A minor of a connectivity function $\lambda$ on set $E$, is a connectivity function $\lambda^{\prime}$ such that $\lambda^{\prime}=\lambda \downarrow A$ for some $A \subseteq E$.

This section follows [2] closely to prove the following theorem:

Theorem 6.2. Let $\lambda$ be a connectivity function on set $E$. If $\lambda$ is an excluded minor for the class of connectivity functions of branch-width at most $k \geq 1$, then $|E| \leq \frac{3^{k+1}-1}{2}$.

\section{Introduction to Branch-Width}

A tree is cubic if every vertex has either degree 1 or degree 3 ; we call a vertex with degree 1 a leaf, and a vertex of a cubic tree that has degree 3 an internal vertex. A partial branch-decomposition of a connectivity function $\lambda$ on a set $E$ is a cubic tree whose leaves are labelled by elements of $E$ and all elements of $E$ label some leaf of the tree. A branch-decomposition is a partial branchdecomposition in which no leaf is labelled by more than one element of $E$. If $T$ is a branch decomposition and $T^{\prime}$ is a subgraph of $T$ whose labelled leaves are labelled by exactly $X \subseteq E$, then we say that $T^{\prime}$ displays $X$. The width of an edge, $e$, of $T$ is $\lambda(X)$ where $X$ is the subsets of $E$ displayed by one of the connected componets of $T \backslash\{e\}$. Note that this is well-defined as $\lambda$ is symmetric. The width of $T$ is the maximum of the widths of the edges.

Definition 6.3. The branch-width of a connectivity function, $\lambda$, is the minimum of the widths of all possible branch decompositions. 
We shall allow our branch-decompositions to contain unlabelled leaves, but note that we can always modify our branch-decomposition, without altering that branch-width, so that we are left with a branch decomposition that contains only labelled leaves.

Consider the following connectivity function $\lambda$ on set $\{a, b, c, d\}: \lambda(\{a\})=1$, $\lambda(\{b\})=1, \lambda(\{c\})=1, \lambda(\{d\})=1, \lambda(\{a, b\})=1, \lambda(\{a, c\})=2, \lambda(\{a, d\})=$ 1 and the remaining values can be found by symmetry.

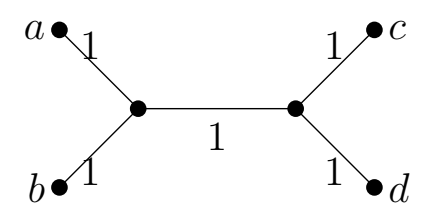

$B_{1}$

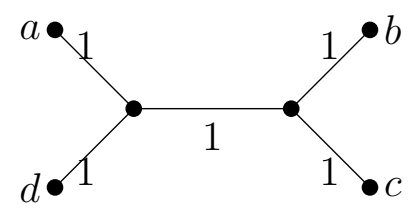

$B_{2}$

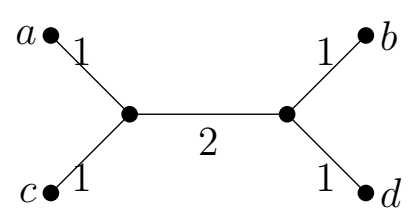

$B_{3}$

All of the above graphs are branch decompositions of $\lambda$. The trees $B_{1}$ and $B_{2}$ have width 1 and $B_{3}$ has width 2 . The branch-width is therefore one and $B_{1}$ and $B_{2}$ are both minimal branch-decompositions (as clearly the branch-width is not zero).

Definition 6.4. Let $\lambda$ be a connectivity function on set $E$ and let $(A, B)$ be a partition of $E$. A branching of $B$ is a partial branch-decomposition of $\lambda$ in which there is a leaf displaying $A$ and no other leaf is multiply labelled. We say that $B$ is $k$-branched if there is a branching, $T$, of $B$ with width less than or equal to $k$.

For example, if we look again at the connectivity function $\lambda$ on set $\{a, b, c, d\}$ such that $\lambda(\{a\})=1, \lambda(\{b\})=1, \lambda(\{c\})=1, \lambda(\{d\})=1, \lambda(\{a, b\})=1$, $\lambda(\{a, c\})=2, \lambda(\{a, d\})=1$ and the remaining values can be found by symmetry, the following would be a branching of $\{c, d\}$ and so we can say that $\{c, d\}$ is 1 -branched, and so $k$-branched for any $k \geq 1$. 


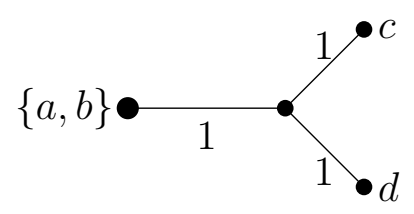

\section{Branch-Width Lemmas}

The following lemma is proved in [2] but is proved again here in slightly more detail.

Lemma 6.5. Let $\lambda$ be a connectivity function on set $E$ with branch-width $k$, and let $(A, B)$ be a partition of $E$ such that $\lambda(A) \leq k$. If $B$ is not $k$ branched, then there is a partition $\left(A_{1}, A_{2}, A_{3}\right)$ of $A$ such that $\lambda\left(A_{i}\right)<\lambda(A)$ for all $i \in\{1,2,3\}$ (note that $A_{i}$ may be empty for som $i \in\{1,2,3$,$\} ).$

Proof. Note that as $\lambda$ has branch-width $k$, every $e \in E$ must have $\lambda(\{e\}) \leq k$. Suppose that for all $\left(A_{1}, A_{2}, A_{3}\right)$ that partition $A$, there is some $\lambda\left(A_{i}\right) \geq \lambda(A)$ for $i \in\{1,2,3\}$.

Claim 6.5.1. If $\left(X_{1}, X_{2}\right)$ is a partition of $E$ such that $\lambda\left(X_{1}\right) \leq k$, then either $\lambda\left(B \cap X_{1}\right) \leq k$ or $\lambda\left(B \cap X_{2}\right) \leq k$.

Proof. Notice that $\left(A \cap X_{1}, A \cap X_{2}, \emptyset\right)$ is a partition of $A$. Therefore $\lambda\left(A \cap X_{1}\right) \geq \lambda(A)$ or $\lambda\left(A \cap X_{2}\right) \geq \lambda(A)$. Without loss of generality suppose that $\lambda\left(A \cap X_{1}\right) \geq \lambda(A)$. Therefore:

$$
\begin{aligned}
\lambda\left(X_{1}\right)+\lambda(A) & \geq \lambda\left(A \cup X_{1}\right)+\lambda\left(A \cap X_{1}\right) \\
& \geq \lambda\left(A \cup X_{1}\right)+\lambda(A) \\
& =\lambda\left(B \cap X_{2}\right)+\lambda(A),
\end{aligned}
$$

where the first inequality follows by submodularity, (2) follows from (1) by noting that $\lambda\left(A \cap X_{1}\right) \geq \lambda(A)$, and (3) follows from (2) by symmetry of $\lambda$ and noting that $A \cup X_{1}=E-\left(B \cap X_{2}\right)$. The claim follows by noting that $\lambda\left(X_{1}\right) \leq k$.

Let $T$ be a branch decomposition of $\lambda$ that is of width $k$. If $|E(T)| \leq 1$ then $B$ will be $k$-branched, so we assume that $T$ contains at least three edges 
(note that $T$ cannot contain two edges).

If $v$ is a vertex of $T$ and $e$ is an edge of $T$, then we use $X_{e, v}$ to denote the set of elements of $E$ that are displayed by the component of $T \backslash e$ that does not contain $v$.

Claim 6.5.2. There is some degree-3 vertex, s, of $T$ such that for every edge, e, of $T$, we have $\lambda\left(X_{e, s} \cap B\right) \leq k$.

Proof. We shall construct an orientation of $T$ as follows: Let $e$ be any edge of $T$ and let $V(\{e\})=\{u, v\}$. If $\lambda\left(X_{e, v} \cap B\right) \leq k$ then orient $e$ from $u$ to $v$, and if $\lambda\left(X_{e, u} \cap B\right) \leq k$ then orient $e$ from $v$ to $u$. As $T$ has branch-width $k$ and so $\lambda\left(X_{e, w}\right) \leq k$ for $w \in\{u, v\}$, by Claim 6.5.1. every edge will receive at least one orientation, and some may have two. Suppose that there is some vertex $v$ such that every vertex in the graph can be connected to $v$ via a directed path. If $v$ has degree 3 , then setting $s=v$ will prove the claim. Suppose now that $v$ has degree 1 and let $f$ be the edge incident with $v$. Let the other vertex incident with $f$ be $v^{\prime}$. From every vertex of $T \backslash f$ there is an oriented path to $v^{\prime}$, and $f$ must be oriented from $v$ to $v^{\prime}$ in $T$, as either $X_{f, v^{\prime}} \cap B=\emptyset$ or $X_{f, v^{\prime}} \cap B=\{v\}$, and therefore $\lambda\left(X_{f, v^{\prime}} \cap B\right) \leq k$. Therefore $v^{\prime}$ is also reachable from every other vertex in the graph via a directed path, and $v^{\prime}$ has degree 3 , so setting $s=v^{\prime}$ gives an $s$ that works.

Now suppose that there is no such vertex. We show that this leads to a contradiction. If no such vertex exists then there is a pair of edges $e$ and $f$, and a vertex, $w$, on the (not directed) path connecting $e$ and $f$ such that neither $e$ nor $f$ is oriented towards $w$. Let $Y_{1}=X_{e, w}, Y_{2}=X_{f, w}$ and $Y_{3}=E-\left(Y_{1} \cup Y_{2}\right)$. As $e$ is oriented away from $w$ we must have $\lambda\left(\left(Y_{2} \cup Y_{3}\right) \cap B\right) \leq k$ and as $f$ is oriented away from $w$ we must have $\lambda\left(\left(Y_{1} \cup Y_{3}\right) \cap B\right) \leq k$. Therefore:

$$
\begin{aligned}
k+k & \geq \lambda\left(\left(Y_{2} \cup Y_{3}\right) \cap B\right)+\lambda\left(\left(Y_{1} \cup Y_{3}\right) \cap B\right) \\
& =\lambda\left(\left(Y_{2} \cup Y_{3}\right) \cap B\right)+\lambda\left(Y_{2} \cup A\right) \\
& \geq \lambda\left(Y_{2} \cap B\right)+\lambda\left(\left(Y_{2} \cup Y_{3}\right) \cup A\right),
\end{aligned}
$$

where (2) follows from (1) by symmetry of $\lambda$ and noting that $E-\left(\left(Y_{1} \cup\right.\right.$ 
$\left.\left.Y_{3}\right) \cap B\right)=Y_{2} \cup A$, and (3) follows from (2) by submodularity and noting that that $\left(\left(\left(Y_{2} \cup Y_{3}\right) \cap B\right) \cup\left(Y_{2} \cup A\right)\right)=\left(\left(Y_{2} \cup Y_{3}\right) \cup A\right)$ and that $\left(\left(\left(Y_{2} \cup Y_{3}\right) \cap B\right) \cap\left(Y_{2} \cup A\right)\right)=\left(Y_{2} \cap B\right)$.

This means that either $\left(Y_{2} \cap B\right) \leq k$ or $\lambda\left(Y_{1} \cap B\right) \leq k$ which contradicts the fact the both $e$ and $f$ are not oriented towards $w$, a contradiction.

Let $s$ be a degree-3 vertex of $T$ that is such that every edge, $e$, of $T$ has $\lambda\left(X_{e, s} \cap B\right) \leq k$. We know that such a vertex exists by Claim 6.5.2. Let $e_{1}, e_{2}$, and $e_{3}$ be the edges of $T$ incident with $s$ and let $X_{e_{i}, s}=X_{i}$ for $i \in\{1,2,3\}$. Recall that we are assuming that for any partition $\left(A_{1}, A_{2}, A_{3}\right)$ of $A$ there is some $A_{i}$ such that $\lambda\left(A_{i}\right) \geq \lambda(A)$. Therefore we may, without loss of generality, assume that $\left(X_{1} \cap A\right) \geq \lambda(A)$. Consider the branching $T^{\prime}$ of $B$ that is constructed by taking a copy of $T$ and keeping only labels from $B$, then subdividing $e_{1}$ with a vertex $w$ and adding a new leaf incident with $w$ whose other vertex is labelled by $A$. This construction is shown below. The green vertices are the vertices from $B$ (these are the labelled vertices in $T^{\prime}$ ) and the new vertex, $w$, and the new leaf are shown in red. The black vertices are unlabelled.

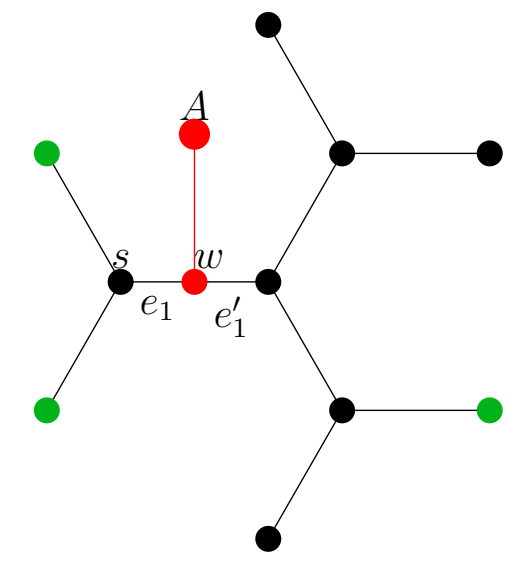

$T^{\prime}$

All edges, except the new edge and $e_{1}$ and $e_{1}^{\prime}$, have width at most $k$ as $\lambda\left(X_{e, s} \cap B\right) \leq k$ for all edges, $e$, of $T$ and the only labels of the vertices are labels from $B$ (except the label $A$, which we are not considering at the moment). We know that $\lambda(A) \leq k$, as this is one of the assumptions in the statement of the lemma, and so the new leaf (indicated by the red edge) has width at most $k$. This means that to show that $T^{\prime}$ is a branching of 
$B$ of width at most $k$, all that remains to check is the widths of $e_{1}$ and $e_{1}^{\prime}$. That is we must check $\lambda\left(X_{1} \cap B\right) \leq k$ and $\lambda\left(\left(X_{2} \cup X_{3}\right) \cap B\right) \leq k$. The first inequality holds by choice of $s$, and the second holds as: $\lambda\left(X_{1}\right)+\lambda(A) \geq$ $\lambda\left(X_{1} \cup A\right)+\lambda\left(X_{1} \cap A\right) \geq \lambda\left(X_{1} \cup A\right)+\lambda(A)$ where the first inequality holds by submodularity and the second by the assumption that $\lambda\left(X_{1} \cap A\right) \geq \lambda(A)$. Therefore $k \geq \lambda\left(X_{1}\right) \geq \lambda\left(X_{1} \cup A\right)=\lambda\left(\left(X_{2} \cup X_{3}\right) \cap B\right)$ and so $e_{1}^{\prime}$ has width at most $k$.

Definition 6.6. Let $f: \mathbb{Z}_{+} \rightarrow \mathbb{Z}_{+}$and $m \in \mathbb{Z}$. We say that a connectivity function, $\lambda$, on set $E$ is $(m, f)$-connected if, for all $A \subseteq E$ such that $\lambda(A)=$ $l<m$, either $|A| \leq f(l)$ or $|E-A| \leq f(l)$.

Recall that the elision of an element, $e$, from a connectivity function, $\lambda$ on a set $E$ with $e \in E$, is defined by $(\lambda \downarrow e)(A)=\min \{\lambda(A), \lambda(A \cup\{e\})\}$ for all $A \subseteq E$.

Lemma 6.7. Let $f: \mathbb{Z}_{+} \rightarrow \mathbb{Z}_{+}$be a non-decreasing function, and let $\lambda$ be $a(m, f)$-connected connectivity function on set $E$. Then for all $e \in E$, the connectivity function $\lambda \downarrow$ e is $(m, f)$-connected.

Proof. Suppose that $(\lambda \downarrow e)(A)=l<m$. Suppose that $(\lambda \downarrow e)(A)=\lambda(A)$. Then $\lambda(A)=l<m$, and so, as $\lambda$ is $(m, f)$ connected, $|A| \leq f(l)$. Suppose that $\lambda \downarrow e=\lambda(A \cup\{e\})$. Then $\lambda(A \cup\{e\})=l<m$ so $|A| \leq|A \cup\{e\}| \leq$ $f(l)$.

For the proof of the next lemma it may be helpful to refer back to Subsection 2.3

Lemma 6.8. If $\lambda$ is an excluded minor for connectivity functions of branchwidth $k$, then $\lambda$ is connected.

Proof. Suppose that $\lambda$ is not connected. Then $\lambda=\lambda_{1} \oplus \lambda_{2}$ for some connectivity functions $\lambda_{1}$ and $\lambda_{2}$ and $\lambda_{1}$ and $\lambda_{2}$ are minors of $\lambda$. Suppose that $\lambda_{1}$ and $\lambda_{2}$ both have branch-width less than or equal to $k$. Let $T_{1}$ be a branch-decomposition of $\lambda_{1}$ with branch width at most $k$ and $T_{2}$ be a branchdecomposition of $\lambda_{2}$ with branch width at most $k$. Now consider the graph obtained by subdividing an edge of $T_{1}$ and subdividing an edge of $T_{2}$ and joining the two new vertices with a new edge, $e$; call this new tree $T$. Clearly 
$e$ has weight 0 in $T$, and $T$ is a branch-decomposition of $\lambda$ with branch-width at most $k$.

Lemma 6.9. Let $\lambda$ be a connectivity function on set $E$. If $\lambda$ be an excluded minor for branch-width $k$ connectivity functions, and $(A, B)$ be a partition of $E$, then it cannot be that both $A$ and $B$ are $k$-branched.

Proof. Let $T_{1}$ be a branching of $A$ of width at most $k$, and let $T_{2}$ be a branching of $B$ of width at most $k$. There is a vertex in $T_{1}$ labelled by $B$. Let $e$ be the edge incident with this vertex. The width of $e$ is equal to $\lambda(A)=\lambda(B)$. Similarly in $T_{2}$ the edge, $f$, incident with the vertex labelled by $A$, has width $\lambda(B)=\lambda(A)$. Let $v_{1}$ be the internal vertex of $T_{1}$ incident with $e$, and let $v_{2}$ be the internal vertex of $T_{2}$ incident with $f$. The graph obtained by joining $T_{1} \backslash\{e\}$ to $T_{2} \backslash\{f\}$ via a new edge $g$, that is incident with vertices $v_{1}$ and $v_{2}$ and has weight $\lambda(A)$ as shown below, gives a branch-decomposition of $\lambda$ that has width $k$; a contradiction.

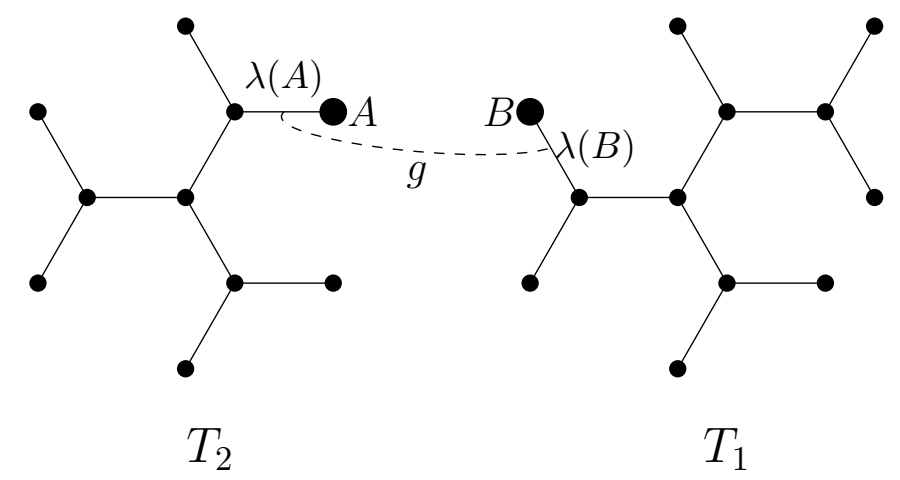

Let $g$ be defined by $g(0)=0$ and $g(n)=3 g(n-1)+1$ for all $n>1$, that is $g(n)=\frac{3^{n}-1}{2}$.

Lemma 6.10. Let $\lambda$ be a connectivity function on set $E$. If $\lambda$ is an excluded minor for the class of connectivity functions of branch-width $k \geq 1$, then $\lambda$ is $(k+1, g)$-connected.

Proof. The proof is by induction. As $\lambda$ is connected, $\lambda$ is $(1, g)$-connected.

For the purposes of induction suppose that $\lambda$ is $(m, g)$-connected for some $m \leq k$. We shall now show that $\lambda$ is $(m+1, g)$-connected. First suppose that 
$\lambda(A)=l<m$. As $\lambda$ is $(m, g)$-connected, either $|A| \leq g(l)$ or $|E-A| \leq g(l)$. Now let $\lambda(A)=m$ and suppose, for the purposes of contradiction, that $|A|>g(m)$ and $|B|>g(m)$ where $B=E-A$. That is, suppose that $|A|>3 g(m-1)+1$ and $|B|>3 g(m-1)+1$.

As $\lambda$ is an excluded minor for connectivity functions of branch-width $k$, it cannot be that both $A$ and $B$ are $k$-branched. Without loss of generality we may assume that $B$ is not $k$-branched. Let $e \in A$, as $\lambda$ is $(m, g)$-connected then so is $\lambda \downarrow e$. As $\lambda \downarrow e$ has branch-width $k$ and $(\lambda \downarrow e)(A-\{e\}) \leq k$, then by Lemma 6.5, either $B$ is $k$-branched in $\lambda \downarrow e$, or there is a partition $\left(A_{1}, A_{2}, A_{3}\right)$ of $A-\{e\}$ such that $(\lambda \downarrow e)\left(A_{i}\right)<(\lambda \downarrow e)(A-\{e\})$ for all $i \in\{1,2,3\}$. We show that it must be that $B$ is $k$-branched in $\lambda \downarrow e$.

Suppose that there were some partition $\left(A_{1}, A_{2}, A_{3}\right)$ of $A-\{e\}$ such that $(\lambda \downarrow$ $e)\left(A_{i}\right)<(\lambda \downarrow e)(A-\{e\})$ for all $i \in\{1,2,3\}$. Then, as $|A|>3 g(m-1)+1$, we have $|A-\{e\}|>3 g(m-1)$ and so for some $i \in\{1,2,3\}$, it must be that $\left|A_{i}\right|>g(m-1)$. As $|B|>3 g(m-1)+1$ we also have $\left|E-A_{i}\right|>3 g(m-1)+1$. Therefore, as $\lambda \downarrow e$ is $(m, g)$-connected, it must be that $(\lambda \downarrow e)\left(A_{i}\right) \geq m=$ $\lambda(A) \geq(\lambda \downarrow e)(A-\{e\})$. Therefore $(\lambda \downarrow e)\left(A_{i}\right) \geq(\lambda \downarrow e)(A-\{e\})$, a contradiction.

Therefore no such partition exists, so $B$ is $k$-branched in $\lambda \downarrow e$.

We show next that this means that $(\lambda \downarrow e)(B) \leq m-1$. For suppose that $(\lambda \downarrow e)(B) \geq m$ and recall that $\lambda(B)=m$. Then $(\lambda \downarrow e)(B)=$ $\min \{\lambda(B), \lambda(B \cup\{e\})\}=\lambda(B)$ so $\lambda(B) \leq \lambda(B \cup\{e\})$. Therefore, by Lemma 2.14. $(\lambda \downarrow e)\left(B^{\prime}\right)=\lambda\left(B^{\prime}\right)$ for all $B^{\prime} \subseteq B$. Therefore $B$ is $k$-branched in $\lambda$, a contradiction. Therefore $(\lambda \downarrow e)(B) \leq m-1$.

As $\lambda \downarrow e$ is $(m, g)$ connected this means that $|B| \leq g(m-1)$ or $|A-\{e\}| \leq$ $g(m-1)$ so $|A| \leq g(m)$, a contradiction.

The following lemma is well-known and a proof can be found in [9]

Lemma 6.11. Every cubic tree, $T$, has an edge, e, such that each of the two connected components of $T \backslash e$ contains at least one-third of the leaves of $T$. 


\subsection{Main Branch-Width Theorem}

Theorem 6.2 Let $\lambda$ be a connectivity function on a set $E$. If $\lambda$ is an excluded minor for the class of connectivity functions of branch-width at most $k \geq 1$, then $|E| \leq \frac{3^{k+1}-1}{2}$.

Proof. First note that if $|E| \leq 3$, then $|E| \leq \frac{3^{k+1}}{2}$. Therefore suppose that $|E|>3$. Let $e \in E$. As $\lambda$ is an excluded minor for connectivity functions of branch-width $k$, it must be that $\lambda \downarrow e$ has branch-width $k$. Let $T$ be a branch decomposition of $\lambda \downarrow e$ that has width $k$. As $T$ contains at least two labelled leaves then, as $T$ is cubic, there is an edge $f$ of $T$ such that $T \backslash f$ displays some sets $X_{1}$ and $X_{2}$ such that $\left|X_{1}\right| \geq \frac{|E-\{e\}|}{3}$ and $\left|X_{2}\right| \geq \frac{|E-\{e\}|}{3}$. Without loss of generality we may assume that $\left|X_{1}\right| \leq\left|X_{2}\right|$. As $X_{1}$ is displayed by $T \backslash f$ the connectivity of $X_{1}$ must be equal to the width of $f$. Therefore $(\lambda \downarrow e)\left(X_{1}\right) \leq k$. As $\lambda$ is $(k+1, g)$ connected, then so is $\lambda \downarrow e$, so $\left|X_{1}\right| \leq g(k)$. Therefore $g(k) \geq \frac{|E|-1}{3}$, and so $|E| \leq 3 g(k)+1=g(k+1)=\frac{3^{k+1}-1}{2}$. 


\section{Bibliography}

[1] Clark, B., Tangles, Trees, and Flowers, M.Sc. thesis, Victoria University of Wellington, 2011.

[2] Geelen, J., Gerards, A., Robertson, N., Whittle, G., On the Excluded Minors for the Matroids of Branch-Width $k$, J. Combin. Ser B 88:261265,2003

[3] Geelen J. and Whittle G, Inequivalent Representations of Matroids over Prime Fields, Advances in Applied Mathematics 51:1-175, 2013

[4] Hall, D., Unavoidable Minors for Connected 2-polymatroids, submitted, 2013

[5] Lehot, P., An Optimal Algorithm to Detect a Line Graph and Output its Root Graph, J. ACM 21:569-575, 1974

[6] Lemos, M., Matroids Having The Same Connectivity Function, Discrete Math 131: 153-161, 1994

[7] Mayhew, D., Newman, M., Whittle, G., Yes, The Missing Axiom of Matroid Theory is Lost Forever, submitted

[8] Mo, S., The Structure of Connectivity Functions, M.Sc. thesis, Victoria University of Wellington, 2013.

[9] Oxley J, Matroid Theory, 2nd ed. Oxford University Press, 2011

[10] Seymour P., Decomposition of Regular Matroids, J. Combin. Ser B 28:305-359, 1980

[11] Seymour, P., Recognising Graphic Matroids, Combinatorica 1:75-78, 1981 
[12] Seymour, P., On the Connectivity Function of a Matroid, J. Combin. Ser B 45: 25-30, 1988

[13] Tutte, W., Connectivity in Matroids, Canad. J. Math. 18:1301-1324, 1966

[14] Tutte, W., Lectures in Matroids, J. Res. Nat. Bur. Stand. 69 B: 1-48, 1965

[15] Whitney, H.,Congruent Graphs and the Connectivity of Graphs, American Journal of Mathematics 54:150-168, 1932

[16] Whittle, G., Duality in Polymatroids and Set Functions, Combinatorics, Probability and Computing 1:275-280, 1992.

[17] Zelinka, B.,Reconstruction a Graph from the Incidence Relation on its Edge Set, Mathematický časopis 22:164-171, 1972 